Metals and Ceramics Division

\title{
A COMPREHENSIVE REVIEW OF THE XRD DATA OF THE PRIMARY AND SECONDARY PHASES PRESENT IN THE BSCCO SUPERCONDUCTOR SYSTEM (Part I: Ca-Sr-Cu OXIDES)
}

\author{
Brian J. Reardon \\ Camden R. Hubbard
}

Date Published - January 1992

NOTICE: This document contains information of a preliminary nature. It is subject to revision or correction and therefore does not represent a final report.

\author{
Prepared for \\ U.S. Department of Energy \\ Assistant Secretary for Conservation and Renewable Energy \\ Office of Transportation Technologies \\ AK 0600000 \\ Prepared by the \\ GAK RIDGE NATIONAL LABORATORY \\ Oak Ridge, Tennessee 37831-6285 \\ managed by \\ MARTIN MARIETTA ENERGY SYSTEMS, INC. \\ for the \\ U.S. DEPARTMENT OF ENERGY \\ under contract DE-AC05-84OR21400)
}

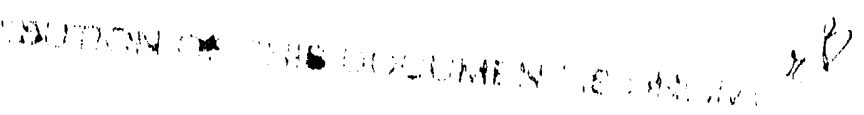


TABLE OF CONTENTS

Page

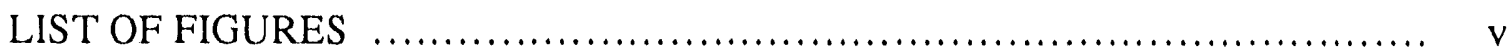

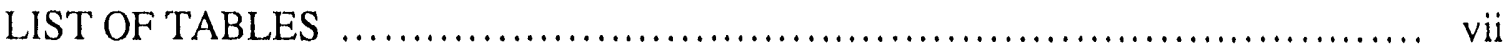

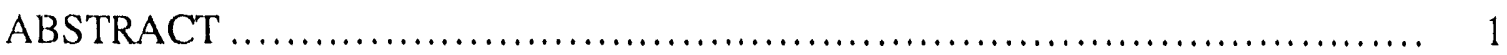

1. INTRODUCTION .......................................................

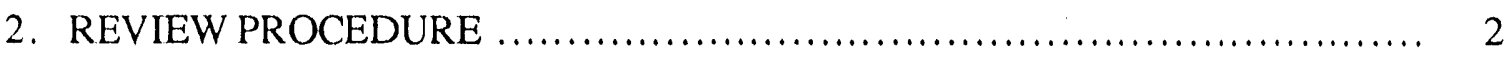

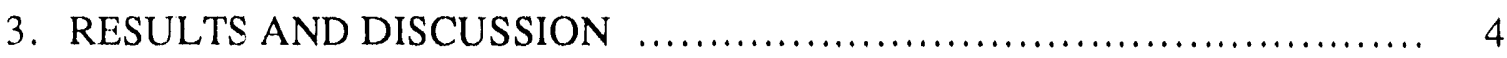

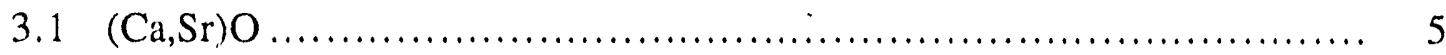

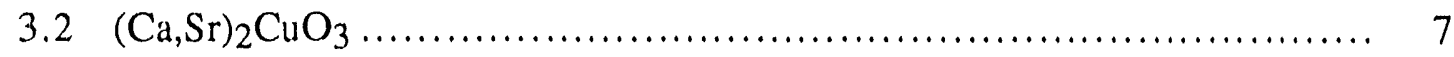

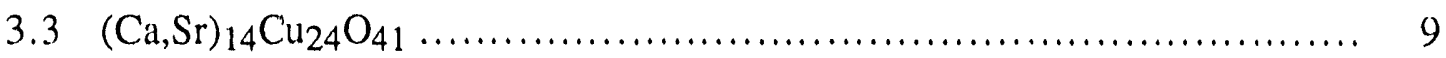

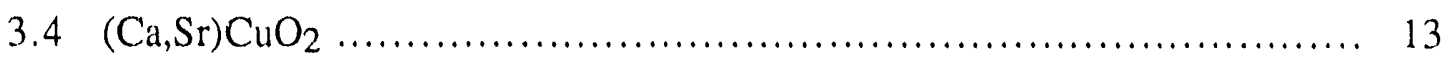

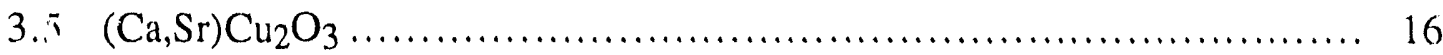

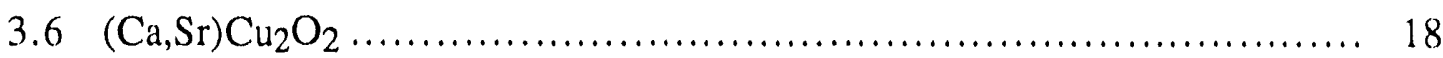

4. CONCLUSIONS ........................................................ 19

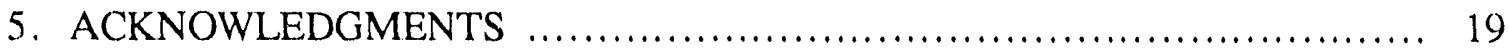

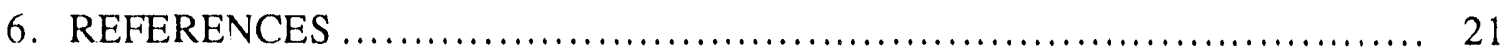

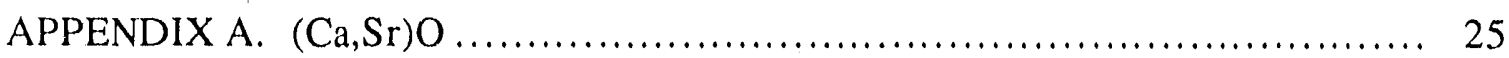

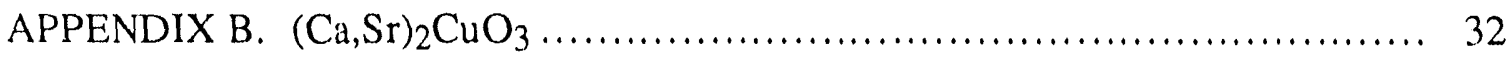

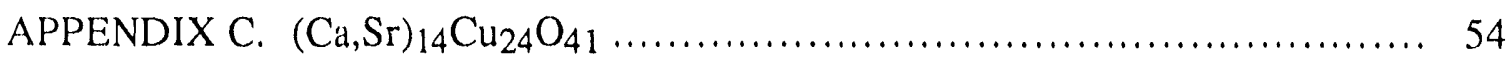

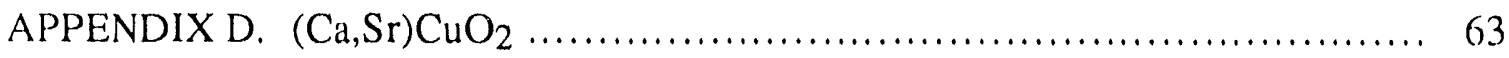

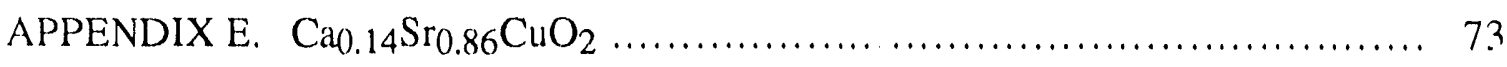

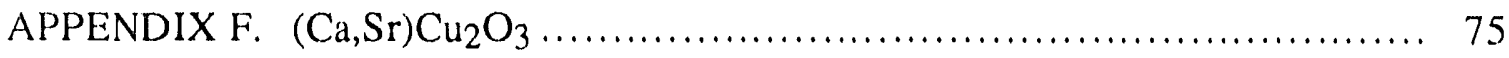

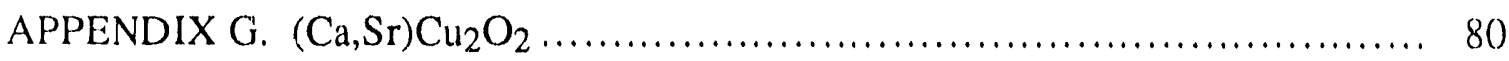




\section{LIST OF FIGURES}

Figure

Page

1 Simulated XRD plots of $\mathrm{Ca}_{2-\mathrm{x}} \mathrm{Sr}_{\mathrm{x}} \mathrm{CuO}_{3}$ for $\mathrm{x}=0,0.5,1.0,1.5,2.0 \ldots \ldots \ldots \ldots 2$ 


\section{LIST OF TABLES}

Table

Page

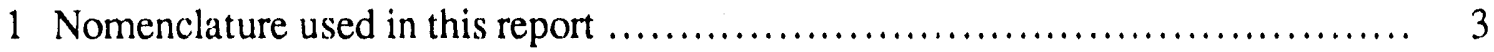

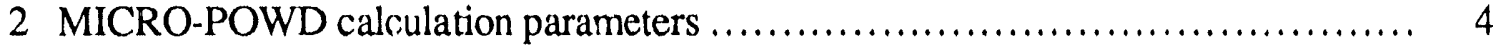

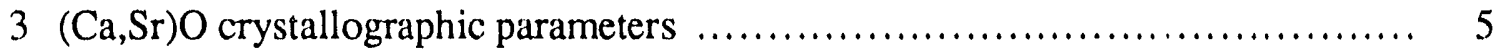

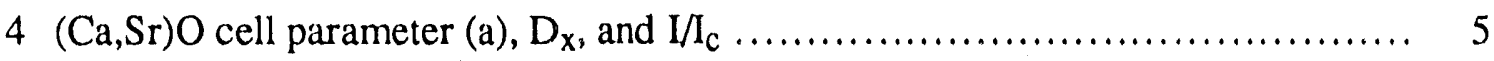

5 Three major lines of simulated (Ca,Sr)O and PDF cards 37-1497 (CaO),

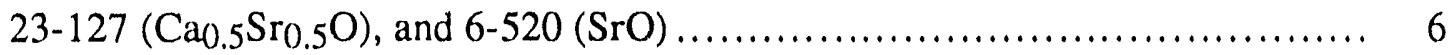

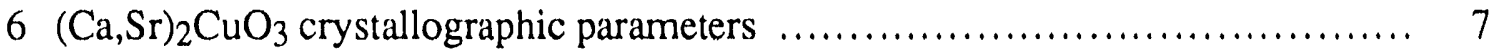

$7(\mathrm{Ca}, \mathrm{Sr})_{2} \mathrm{CuO}_{3}$ unit cell parameters $(\mathrm{a}), \mathrm{D}_{\mathrm{x}}$, and $\mathrm{I} / \mathrm{I}_{\mathrm{c}} \ldots \ldots \ldots \ldots \ldots \ldots \ldots \ldots \ldots \ldots \ldots$

8 Three major lines of simulated $\mathrm{Ca}_{2} \mathrm{CuO}_{3}, \mathrm{CaSrCuO}_{3}, \mathrm{Sr}_{2} \mathrm{CuO}_{3}$, and PDF cards: $34-282\left(\mathrm{Ca}_{2} \mathrm{CuO}_{3}\right)$ and $34-283\left(\mathrm{Sr}_{2} \mathrm{CuO}_{3}\right) \ldots \ldots \ldots \ldots \ldots \ldots \ldots \ldots . \ldots$

$9(\mathrm{Ca}, \mathrm{Sr})_{14} \mathrm{Cu}_{24} \mathrm{O}_{41}$ crystallographic parameters ............................ 10

$10(\mathrm{Ca}, \mathrm{Sr})_{14} \mathrm{Cu}_{24} \mathrm{O}_{41}$ unit cell parameters, $\mathrm{D}_{\mathbf{x}}$, and $\mathrm{I} / \mathrm{I}_{\mathrm{c}} \ldots \ldots \ldots \ldots \ldots \ldots \ldots \ldots \ldots \ldots \ldots$

11 Three major lines of simulated $\mathrm{Sr}_{14} \mathrm{Cu}_{24} \mathrm{O}_{41}, \mathrm{Ca}_{3} \mathrm{Sr}_{11} \mathrm{Cu}_{24} \mathrm{O}_{41}$, $\mathrm{Ca}_{5} \mathrm{Sr}_{9} \mathrm{Cu}_{24} \mathrm{O}_{41}, \mathrm{Ca}_{7} \mathrm{Sr}_{7} \mathrm{Cu}_{24} \mathrm{O}_{41}$, observed $\mathrm{Sr}_{14} \mathrm{Cu}_{24} \mathrm{O}_{41}$ and PDF cards

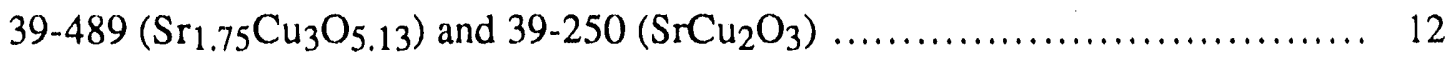

$12(\mathrm{Ca}, \mathrm{Sr})_{2} \mathrm{CuO}_{2}$ crystallographic parameters ................................. 13

$13(\mathrm{Ca}, \mathrm{Sr}) \mathrm{CuO}_{2}$ and $\mathrm{Ca} 0.86 \mathrm{Sr}_{0.14} \mathrm{CuO}_{2}$ unit cell parameters, $\mathrm{D}_{\mathrm{x}}$, and $\mathrm{I} / \mathrm{I}_{\mathrm{c}} \ldots \ldots \ldots \ldots .14$

14 Three main peaks of simulated $\mathrm{SrCuO}_{2}, \mathrm{Ca}_{0.3} \mathrm{Sr}_{0.7} \mathrm{CuO}_{2}, \mathrm{Ca}_{0.25} \mathrm{Sr}_{0.75} \mathrm{CuO}_{2}$, and PDF cards: $39-1492\left(\mathrm{SrCuO}_{2}\right)$ and $38-1179\left(\mathrm{SrCuO}_{2}\right) \ldots \ldots \ldots \ldots \ldots \ldots . . \ldots$

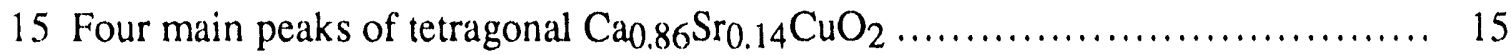

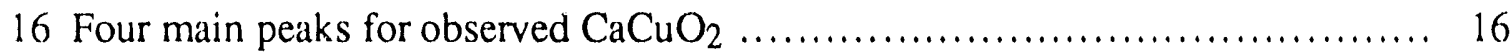

$17(\mathrm{Ca}, \mathrm{Sr}) \mathrm{Cu}_{2} \mathrm{O}_{3}$ crystallographic parameters and $\mathrm{L} / \mathrm{I}_{\mathrm{c}}$ (for all four models: $\left.a=9.942 ; b=4.0786 ; c=3.459 ; D_{x}=5.094\right) \ldots \ldots \ldots \ldots \ldots \ldots \ldots \ldots \ldots \ldots \ldots$

18 Three strongest lines for the four different structure models of $\mathrm{CaCu}_{2} \mathrm{O}_{3}$ presented by Hoppe and PDF card 34-284 $\left(\mathrm{CaCu}_{2} \mathrm{O}_{3}\right) \ldots \ldots \ldots \ldots \ldots \ldots \ldots \ldots \ldots$

$19 \mathrm{SrCu}_{2} \mathrm{O}_{2}$ crystallographic parameters .................................... 18

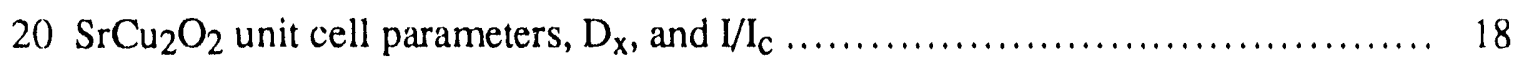

21 Three main peaks of $\mathrm{SrCu}_{2} \mathrm{O}_{2}$ and PDF card 38-1178 $\left(\mathrm{SrCu}_{2} \mathrm{O}_{2}\right) \ldots \ldots \ldots \ldots \ldots$

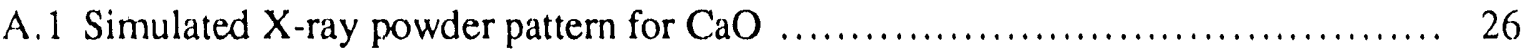

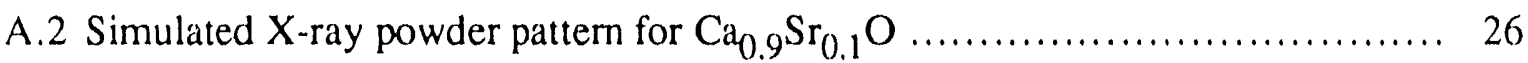




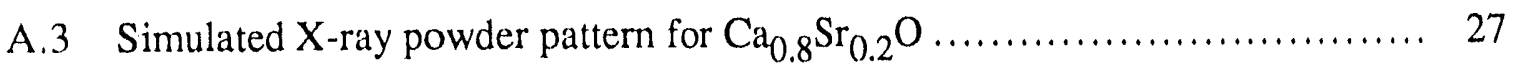

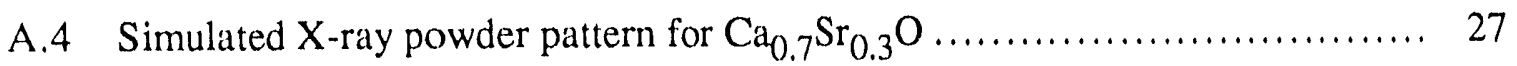

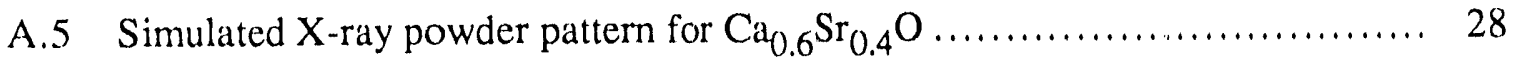

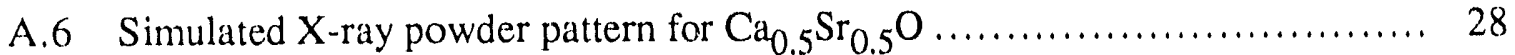

A.7 Simulated $\mathrm{X}$-ray powder pattern for $\mathrm{Ca}_{0.4} \mathrm{Sr}_{0.6} \mathrm{O} \ldots \ldots \ldots \ldots \ldots \ldots \ldots \ldots \ldots \ldots \ldots$

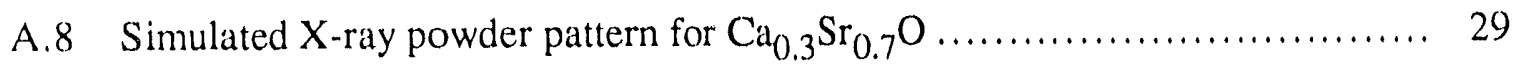

A.9 Simulated X-ray powder pattern for $\mathrm{Ca}_{0.2} \mathrm{Sr}_{0.8} \mathrm{O} \ldots \ldots \ldots \ldots \ldots \ldots \ldots \ldots \ldots \ldots$. 30

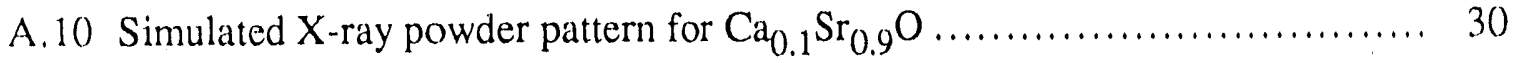

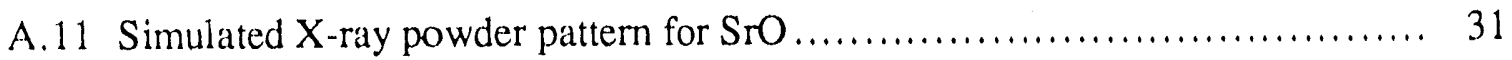

B.1 Simulated X-ray powder pattern for $\mathrm{Ca}_{2} \mathrm{CuO}_{3} \ldots \ldots \ldots \ldots \ldots \ldots \ldots \ldots \ldots \ldots . \ldots \ldots$

B.2 Simulated $\mathrm{X}$-ray powder pattern for $\mathrm{Ca}_{1.9} \mathrm{Sr}_{0.1} \mathrm{CuO}_{3} \ldots \ldots \ldots \ldots \ldots \ldots \ldots \ldots$

B.3 Simulated X-ray powder pattern for $\mathrm{Ca}_{1.8} \mathrm{Sr}_{0.2} \mathrm{CuO}_{3} \ldots \ldots \ldots \ldots \ldots \ldots \ldots \ldots$

B.4 Simulated $\mathrm{X}$-ray powder pattern for $\mathrm{Ca}_{1.7} \mathrm{Sr}_{0.3} \mathrm{CuO}_{3} \ldots \ldots \ldots \ldots \ldots \ldots \ldots \ldots \ldots$

B.5 Simulated $\mathrm{X}$-ray powder pattern for $\mathrm{Ca}_{1.6} \mathrm{Sr}_{0.4} \mathrm{CuO}_{3} \ldots \ldots \ldots \ldots \ldots \ldots \ldots \ldots . \ldots \ldots$

B.6 Simulated $\mathrm{X}$-ray powder pattern for $\mathrm{Ca}_{1.5} \mathrm{Sr}_{0.5} \mathrm{CuO}_{3} \ldots \ldots \ldots \ldots \ldots \ldots \ldots \ldots . \ldots \ldots$

B.7 Simulated X-ray powder pattern for $\mathrm{Ca}_{1.4} \mathrm{Sr}_{0.6} \mathrm{CuO}_{3} \ldots \ldots \ldots \ldots \ldots \ldots \ldots \ldots . . \ldots \ldots$

B.8 Simulated $\mathrm{X}$-ray powder pattern for $\mathrm{Ca}_{1.3} \mathrm{Sr}_{0.7} \mathrm{CuO}_{3} \ldots \ldots \ldots \ldots \ldots \ldots \ldots \ldots$.

B.9 Simulated X-ray powder pattern for $\mathrm{Ca}_{1.2} \mathrm{Sr}_{0.8} \mathrm{CuO}_{3} \ldots \ldots \ldots \ldots \ldots \ldots \ldots \ldots, 41$

B.10 Simulated $\mathrm{X}$-ray powder pattern for $\mathrm{Ca}_{1.1} \mathrm{Sr}_{0.9} \mathrm{CuO}_{3} \ldots \ldots \ldots \ldots \ldots \ldots \ldots . \ldots 42$

B.11 Simulated X-ray powder pattern for $\mathrm{CaSrCuO}_{3} \ldots \ldots \ldots \ldots \ldots \ldots \ldots \ldots \ldots . \ldots 43$

B.12 Simulated $\mathrm{X}$-ray powder pattern for $\mathrm{Ca}_{0.9} \mathrm{Sr}_{1.1} \mathrm{CuO}_{3} \ldots \ldots \ldots \ldots \ldots \ldots \ldots . \ldots 4$

B.13 Simulated $\mathrm{X}$-ray powder pattern for $\mathrm{Ca}_{0.8} \mathrm{Sr}_{1.2} \mathrm{CuO}_{3} \ldots \ldots \ldots \ldots \ldots \ldots \ldots . . \ldots 5$

B.14 Simulated $\mathrm{X}$-ray powder pattern for $\mathrm{Ca}_{0.7} \mathrm{Sr}_{1.3} \mathrm{CuO}_{3} \ldots \ldots \ldots \ldots \ldots \ldots \ldots \ldots, 46$

B.15 Simulated $\mathrm{X}$-ray powder pattern for $\mathrm{Ca}_{(0.6} \mathrm{Sr}_{1.4} \mathrm{CuO}_{3} \ldots \ldots \ldots \ldots \ldots \ldots \ldots . . \ldots 4$

B.16 Simulated $\mathrm{X}$-ray powder pattern for $\mathrm{Ca}_{0.5} \mathrm{Sr}_{1.5} \mathrm{CuO}_{3} \ldots \ldots \ldots \ldots \ldots \ldots \ldots . . \ldots 4$

B.17 Simulated $\mathrm{X}$-ray powder pattern for $\mathrm{Ca}_{0.4} \mathrm{Sr}_{1.6} \mathrm{CuO}_{3} \ldots \ldots \ldots \ldots \ldots \ldots \ldots . . \ldots 4$

B.18 Simulated $\mathrm{X}$-ray powder pattern for $\mathrm{Ca}_{0.3} \mathrm{Sr}_{1.7} \mathrm{CuO}_{3} \ldots \ldots \ldots \ldots \ldots \ldots \ldots \ldots$. . . . . . . . . .

B.19 Simulated X-ray powder pattern for $\mathrm{Ca}_{0.2} \mathrm{Sr}_{1.8} \mathrm{CuO}_{3} \ldots \ldots \ldots \ldots \ldots \ldots \ldots \ldots . \ldots \ldots$

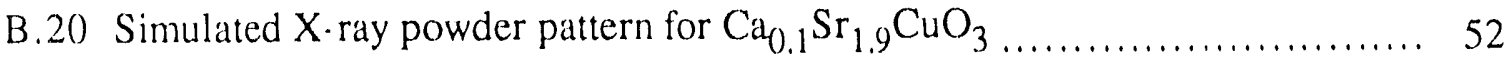

B.21 Simulated X-ray powder pattern for $\mathrm{Sr}_{2} \mathrm{CuO}_{3} \ldots \ldots \ldots \ldots \ldots \ldots \ldots \ldots \ldots \ldots . \ldots 3$

C. 1 Simulated X-ray powder pattern for $\mathrm{Sr}_{14} \mathrm{Cu}_{24} \mathrm{O}_{41} \ldots \ldots \ldots \ldots \ldots \ldots \ldots \ldots \ldots$

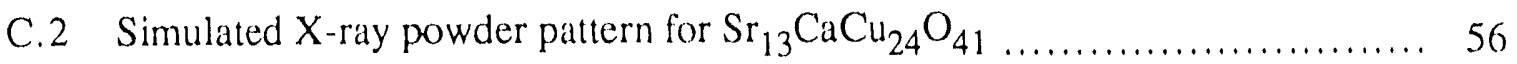

C.3 Simulated $\mathrm{X}$-ray powder pattern for $\mathrm{Sr}_{12} \mathrm{Ca}_{2} \mathrm{Cu}_{24} \mathrm{O}_{41} \ldots \ldots \ldots \ldots \ldots \ldots \ldots . \ldots 57$

C.4 Simulated X-ray powder pattern for $\mathrm{Sr}_{11} \mathrm{Ca}_{3} \mathrm{Cu}_{24} \mathrm{O}_{41} \ldots \ldots \ldots \ldots \ldots \ldots \ldots \ldots$ 
C. 5 Simulated X-ray powder pattern for $\mathrm{Sr}_{10} \mathrm{Ca}_{4} \mathrm{Cu}_{24} \mathrm{O}_{41} \ldots \ldots \ldots \ldots \ldots \ldots \ldots \ldots \ldots$................. 59

C.6 Simulated X-ray powder pattern for $\mathrm{Sr}_{9} \mathrm{Ca}_{5} \mathrm{Cu}_{24} \mathrm{O}_{41} \ldots \ldots \ldots \ldots \ldots \ldots \ldots \ldots \ldots$

C.7 Simulated X-ray powder pattern for $\mathrm{Sr}_{8} \mathrm{Ca}_{6} \mathrm{Cu}_{24} \mathrm{O}_{41} \ldots \ldots \ldots \ldots \ldots \ldots \ldots \ldots \ldots \ldots \ldots$

C.8 Simulated X-ray powder pattern for $\mathrm{Sr}_{7} \mathrm{Ca}_{7} \mathrm{Cu}_{24} \mathrm{O}_{41} \ldots \ldots \ldots \ldots \ldots \ldots \ldots \ldots . \ldots 2$

D.1 Simulated X-ray powder pattern for $\mathrm{SrCuO}_{2} \ldots \ldots \ldots \ldots \ldots \ldots \ldots \ldots \ldots \ldots \ldots \ldots . \ldots . \ldots \ldots$

D.2 Simulated $\mathrm{X}$-ray powder pattern for $\mathrm{Sr}_{0.9} \mathrm{Ca}_{0.1} \mathrm{CuO}_{2} \ldots \ldots \ldots \ldots \ldots \ldots \ldots \ldots \ldots$

D.3 Simulated $\mathrm{X}$-ray powder pattern for $\mathrm{Sr}_{0.8} \mathrm{Ca}_{0.2} \mathrm{CuO}_{2} \ldots \ldots \ldots \ldots \ldots \ldots \ldots \ldots \ldots, 66$

D.4 Simulated X-ray powder pattern for $\mathrm{Sr}_{0.7} \mathrm{Ca}_{0.3} \mathrm{CuO}_{2} \ldots \ldots \ldots \ldots \ldots \ldots \ldots \ldots \ldots \ldots$

D.5 Simulated $\mathrm{X}$-ray powder pattern for $\mathrm{Sr}_{0.6} \mathrm{Ca}_{0.4} \mathrm{CuO}_{2} \ldots \ldots \ldots \ldots \ldots \ldots \ldots \ldots \ldots . \ldots 8$

D.6 Simulated $\mathrm{X}$-ray powder pattern for $\mathrm{Sr}_{0.5} \mathrm{Ca}_{0.5} \mathrm{CuO}_{2} \ldots \ldots \ldots \ldots \ldots \ldots \ldots \ldots \ldots$

D.7 Simulated $X$-ray powder pattern for $\mathrm{Sr}_{0.4} \mathrm{Ca}_{0.6} \mathrm{CuO}_{2} \ldots \ldots \ldots \ldots \ldots \ldots \ldots \ldots \ldots \quad 70$

D.8 Simulated $\mathrm{X}$-ray powder pattern for $\mathrm{Sr}_{0.3} \mathrm{Ca}_{0.7} \mathrm{CuO}_{2} \ldots \ldots \ldots \ldots \ldots \ldots \ldots \ldots \ldots, 71$

D.9 Simulated $X$-ray powder pattern for $\mathrm{Sr}_{0.25} \mathrm{Ca}_{0.75} \mathrm{CuO}_{2} \ldots \ldots \ldots \ldots \ldots \ldots \ldots \ldots . \ldots 2$

E.1 Simulated $\mathrm{X}$-ray powder pattern for $\mathrm{Ca}_{0.14} \mathrm{Sr}_{0.86} \mathrm{CuO}_{2} \ldots \ldots \ldots \ldots \ldots \ldots \ldots \ldots . . \ldots 4$

F.1 Simulated X-ray powder pattern for $\mathrm{CaCu}_{2} \mathrm{O}_{3}$ Model $1 \ldots \ldots \ldots \ldots \ldots \ldots \ldots \ldots . \ldots 6$

F.2 Simulated $\mathrm{X}$-ray powder pattern for $\mathrm{CaCu}_{2} \mathrm{O}_{3}$ Model $2 \ldots \ldots \ldots \ldots \ldots \ldots \ldots \ldots . \ldots 7$

F.3 Simulated $\mathrm{X}$-ray powder pattern for $\mathrm{CaCu}_{2} \mathrm{O}_{3}$ Model $3 \ldots \ldots \ldots \ldots \ldots \ldots \ldots \ldots . . \ldots$

F.4 Simulated $\mathrm{X}$-ray powder pattern for $\mathrm{CaCu}_{2} \mathrm{O}_{3}$ Model $4 \ldots \ldots \ldots \ldots \ldots \ldots \ldots \ldots . \ldots$

G.1 Simulated X-ray powder pattern for $\mathrm{SrCu}_{2} \mathrm{O}_{2} \ldots \ldots \ldots \ldots \ldots \ldots \ldots \ldots \ldots \ldots \ldots$ 


\title{
A COMPREHENSIVE REVIEW OF THE XRD DATA OF THE PRIMARY AND SECONDARY PHASES PRESENT IN THE BSCCO SUPERCONDUCTOR SYSTEM \\ (Part I: Ca-Sr-Cu OXIDES) ${ }^{*}$
}

\author{
B. J. Reardon ${ }^{\dagger}$ and C. R. Hubbard $\ddagger$
}

\begin{abstract}
$\mathrm{X}$-ray powder patterns for the phases in the $\mathrm{CaO}-\mathrm{SrO}-\mathrm{CuO}$ ternary system, along with the corresponding crystal structures, were obtained from the literature and from the Powder Diffraction File (PDF). Available X-ray diffraction (XRD) patterns were compared with each other and, when possible, with a simulated pattern for each phase, yielding a recommended reference pattern. The simulated powder patterns presented here deal with the phases found within the $(\mathrm{Ca}, \mathrm{Sr}) \mathrm{O}$, $(\mathrm{Ca}, \mathrm{Sr})_{2} \mathrm{CuO}_{3},(\mathrm{Ca}, \mathrm{Sr})_{14} \mathrm{Cu}_{24} \mathrm{O}_{41},(\mathrm{Ca}, \mathrm{Sr}) \mathrm{CuO}_{2},(\mathrm{Ca}, \mathrm{Sr}) \mathrm{Cu}_{2} \mathrm{O}_{3}$, and $(\mathrm{Ca}, \mathrm{Sr}) \mathrm{Cu}_{2} \mathrm{O}_{2}$ solid solution series and are recommended for the PDF.
\end{abstract}

\section{INTRODUCTION}

The discovery of superconductivity in the $\mathrm{Bi}-\mathrm{Pb}-\mathrm{Sr}-\mathrm{Ca}-\mathrm{Cu}-\mathrm{O}$ (BSCCO) system by Maeda et al. ${ }^{1}$ has spurred intense research in the X-ray characterization of these materials. As a consequence, significant research has been conducted in the study of the phase diagrams of these four- and five-component systems. ${ }^{2-8}$ Unfortunately, accurate X-ray powder patterns and crystallographic data for many of the minor phases present in these systems are lacking. This is especially true in the case of the solid solution series whose patterns are significantly different from the corresponding end members (see Fig. 1). The lack of corresponding reference patterns in the Powder Diffraction File (PDF) severely limits identification of these phases by X-ray diffraction (XRD). Reference XRD data for the

\footnotetext{
${ }^{*}$ Research sponsored by the U. S. Department of Energy, Assistant Secretary for Conservation and Renewable Energy, Office of Utility Technologies, Office of Energy Management/Advanced Utility Concepts - Superconducting Technology Program for Electric Systems and by the Office of Transportation Technologies as part of the High Temperature Materials Laboratory User Program, under contract DE-AC()5-84OR2140() managed by Martin Marietta Energy Systems, Inc.

${ }^{\dagger}$ Alfred University, Binns-Merril Hall, Alfred, New York.

¥Martin Marietta Energy Systems, Inc., Oak Ridge Natl. Lab., Oak Ridge, Tennessee.
} 
BSCCO superconductor phases 2201, 2212, and 2223 were published by Matheis and Snyder. ${ }^{9}$ This article (part 1 of a series extensively reviewing the XRD data of the BSCCO system) focuses on the phases found in the $\mathrm{CaO}-\mathrm{SrO}-\mathrm{CuO}$ ternary system.

ORNL DWG 92-5017

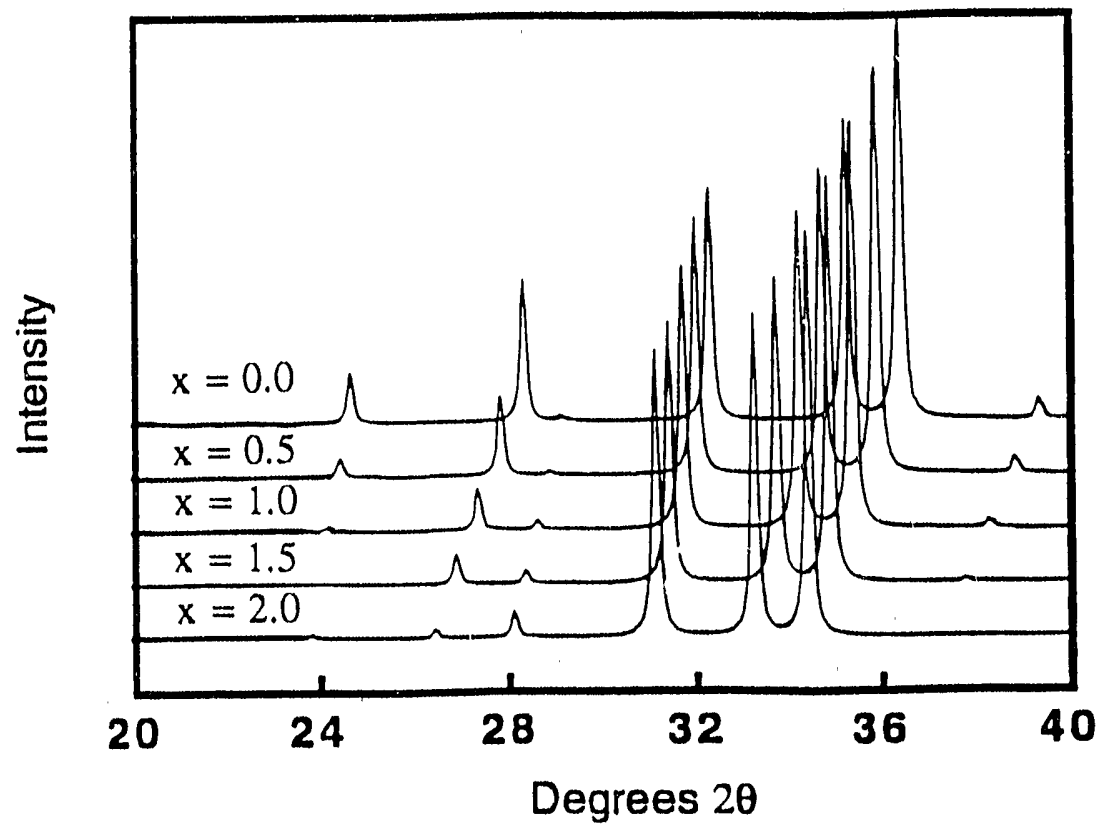

Fig. 1. Simulated XRD plots of $\mathrm{Ca}_{2-x} \mathrm{Sr}_{x} \mathrm{CuO}_{3}$ for $x=0,0.5,1.0,1.5,2.0$.

A complete listing of the XRD patterns for the phases and solid solutions discussed in this report are reported in Appendices A through G. Table 1 presents the definitions of the abbreviations used here.

\section{REVIEW PROCEDURE}

An extensive review of the CaO-SrO-CuO ternary system phases and corresponding $X R D$ data was conducted. Data utilized in this review included that obtained from literature surveys, the PDF, and private communications. Once an adequate understanding of the possible phases, solid solution series, crystal structure, and high-temperature polymorphs 
Table 1. Nomenclature used in this report

\begin{tabular}{ll}
\hline$*$ & $\begin{array}{l}\text { a rating given to powder patterns by the JCPDS, } \\
\text { symbolizing high quality and reliability }\end{array}$ \\
$\Delta 2 \theta$ & change in $2 \theta$ \\
$2 \theta$ & two times the angle of diffraction \\
2201 & $\mathrm{Bi}_{2} \mathrm{Sr}_{2} \mathrm{CuO}_{6}$ \\
2212 & $\mathrm{Bi}_{2} \mathrm{Sr}_{2} \mathrm{CaCu}_{2} \mathrm{O}_{8}$ \\
2223 & $\mathrm{Bi}_{2} \mathrm{Sr}_{2} \mathrm{Ca}_{2} \mathrm{Cu}_{3} \mathrm{O}_{10}$ \\
a & an axis that defines a unit cell (in $\AA$ ) \\
b & an axis that defines a unit cell (in $\AA$ ) \\
Biso & isotropic thermal parameter \\
$\mathrm{BSCCO}$ & Bi-Pb-Sr-Ca-Cu-O \\
c & an axis that defines a unit cell (in $\AA$ ) \\
D & density (in g/cm $\left.{ }^{3}\right)$ \\
d & d-spacing (in $\AA$ ) \\
D & calculated density (in g/cm3) \\
h k $\ell$ & notation used to define crystallographic planes \\
I & intensity \\
I(DS) & relative Debye-Scherrer intensity \\
I(INT) & relative integrated peak intensity \\
I(PK) & relative peak intensity \\
I/I & intensity of the $100 \%$ peak/intensity of the $100 \%$ \\
Ircl & relative intensity \\
Nobs & number of observed peaks \\
Nposs & number of possible peaks \\
occ. & lattice site occupancy \\
\hline &
\end{tabular}


was acquired, the patterns were simulated for all the phases (including solid solutions) using the personal computer (PC) version of MICRO-POWD. ${ }^{10}$ The parameters used in these calculations are listed in Table 2 . The simulated patterns and patterns acquired from the literature search and experiments were compared. Figures of merit (FOMs) for all patterns were calculated using LSQ90, a version of the lattice parameter refinement program of Evans, Appleman, and Handwerker. ${ }^{11}$ The comparisons of FOMs and simulated patterns with experimental data resulted in the suggestion of a set of recommended reference powder patterns.

Table 2. MICRO-POWD calculation parameters

1. Program: MICRO-POWD Version: 1.

2. Scattering factors as provided by MICRO-POWD for: $\mathrm{O}^{2-}, \mathrm{Sr}^{2+}$, $\mathrm{Ca}^{2+}, \mathrm{Cu}^{2+}$.

3. Anomolous scattering factors as provided by MICRO-POWD for: $\mathrm{O}, \mathrm{Sr}, \mathrm{Ca}, \mathrm{Cu}$.

4. $\mathrm{B}_{\text {iso }}($ Oxygen $)=2.5 ; \mathrm{B}_{\text {iso }}$ (all others $)=1$ unless structure reference provided thermal parameters.

5. No incident or diffracted beam monochromators.

6. $\theta$ compensating variable divergence slit was not selected.

7. $\mathrm{CuK} \alpha 1$ radiation was selected $(\lambda=1.5405981 \AA)$.

8. For relative peak intensities, a Cauchy profile was selected.

9. The NBS width table provided with MICRO-POWD supplied the full width at half maximum (FWHM).

\section{RESULTS AND DISCUSSION}

The phases and solid solutions present in the CaO-SrO-CuO ternary system have been extensively studied by a number of authors. $2-4,6,7$ The phases of primary concern when synthesizing BSCCO materials are: $(\mathrm{Ca}, \mathrm{Sr}) \mathrm{O},(\mathrm{Ca}, \mathrm{Sr})_{2} \mathrm{CuO}_{3},(\mathrm{Ca}, \mathrm{Sr})_{14} \mathrm{Cu}_{24} \mathrm{O}_{41}$, $(\mathrm{Ca}, \mathrm{Sr}) \mathrm{CuO}_{2}, \mathrm{Ca}_{() .86} \mathrm{Sr}_{(\mathrm{U}, 14} \mathrm{CuO}_{2}$, and $(\mathrm{Ca}, \mathrm{Sr}) \mathrm{Cu}_{2} \mathrm{O}_{3}$. 


\section{1 (Ca,Sr)O}

$\mathrm{CaO}$ and $\mathrm{SrO}$ have been shown to participate in a complete solid solution series across the entire binary phase system. ${ }^{2}$ Furthermore, the PDF has three cards that provide information on the (Ca,Sr)O system: $37-1497$ (ref. 12) and 6-520 (ref. 13) provide powder patterns for $\mathrm{CaO}$ and $\mathrm{SrO}$, respectively, while 23-127 (ref. 14) provides a powder pattern of the $\mathrm{Ca}_{0.5} \mathrm{Sr}_{0.5} \mathrm{O}$ phase in the solid solution series.

Table 3 displays the crystallographic data for $\mathrm{CaO}$ and $\mathrm{SrO}$ as used in the calculations of the simulated patterns across the solid solution scries. A linear change in the cell

Table 3. (Ca,Sr)O crystallographic parameters $12,13,15$

\begin{tabular}{lclrlll}
\hline Struct. & S.G. & Atom & $\begin{array}{r}\text { Occ. } \\
0<\mathrm{x}<1\end{array}$ & $\mathrm{x}$ & $\mathrm{y}$ & $\mathrm{z}$ \\
\hline \multirow{3}{*}{ Cubic } & $\mathrm{Fm} 3 \mathrm{~m}$ & $\mathrm{O}(1)$ & 4 & 0.5 & 0.5 & 0.5 \\
& $(225)$ & $\mathrm{Ca}(1)$ & $4-4 \mathrm{x}$ & 0.0 & 0.0 & 0.0 \\
& & $\mathrm{Sr}$ & $4 \mathrm{x}$ & 0.0 & 0.0 & 0.0 \\
\hline
\end{tabular}

parameters was assumed for this solid solution series, as indicated in Table 4. Table 5 displays the simulated XRD data for the three main peaks of selected phases across the solid solution series as well as the patterns for PDF cards 37-1497 (CaO), 23-127

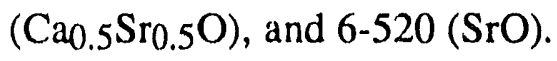

Table 4. (Ca,Sr)O cell parameter (a), ${ }^{12,13}$ $\mathrm{D}_{\mathrm{X}}$, and $\mathrm{I} / \mathrm{I}_{\mathrm{C}}$

\begin{tabular}{|c|c|c|c|}
\hline Phase & a & $\mathrm{D}_{\mathrm{x}}$ & $\mathrm{I} / \mathrm{I}_{\mathrm{c}}$ \\
\hline $\mathrm{SrO}$ & 5.16000 & 5.009 & 7.405 \\
\hline $\mathrm{Sr}_{0.9} \mathrm{Ca}_{0.1} \mathrm{O}$ & 5.12506 & 4.877 & 7.115 \\
\hline $\mathrm{Sr}_{0.8} \mathrm{Ca} 0.2 \mathrm{O}$ & 5.09012 & 4.739 & 6.823 \\
\hline $\mathrm{Sr}_{0.7} \mathrm{Ca}_{0.3} \mathrm{O}$ & 5.05518 & 4.593 & 6.531 \\
\hline $\mathrm{Sr}_{0.6} \mathrm{Ca} 0.4 \mathrm{O}$ & 5.02024 & 4.441 & 6.237 \\
\hline $\mathrm{Sr}_{0.5} \mathrm{Ca} 0.5 \mathrm{O}$ & 4.98529 & 4.280 & 5.942 \\
\hline $\mathrm{Sr}_{0.4} \mathrm{Ca}_{0.6} \mathrm{O}$ & 4.95036 & 4.111 & 5.646 \\
\hline $\mathrm{Sr}_{0.3} \mathrm{Ca} 0.7 \mathrm{O}$ & 4.91541 & 3.933 & 5.348 \\
\hline $\mathrm{Sr}_{0.2} \mathrm{Ca}_{0.8} \mathrm{O}$ & 4.88047 & 3.747 & 5.050 \\
\hline $\mathrm{Sr}_{0.1} \mathrm{Ca} 0.9 \mathrm{O}$ & 4.84553 & $3.55 i$ & 4.751 \\
\hline $\mathrm{CaO}$ & 4.81059 & 3.345 & 4.451 \\
\hline
\end{tabular}


Table 5. Three major lines of simulated $(\mathrm{Ca}, \mathrm{Sr}) \mathrm{O}$

and PDF cards 37-1497 (CaO), 23-127

$\left(\mathrm{Ca}_{0.5} \mathrm{Sr}_{0.5} \mathrm{O}\right)$, and 6-520 (SrO)

\begin{tabular}{|c|c|c|c|c|c|c|}
\hline \multirow[b]{2}{*}{$\underline{h k \ell}$} & \multicolumn{2}{|c|}{$\mathrm{CaO}$} & \multicolumn{2}{|c|}{$\mathrm{Ca}_{0.5} \mathrm{Sr}_{0.5} \mathrm{O}$} & \multicolumn{2}{|c|}{$\mathrm{SrO}$} \\
\hline & $d$ & 1 & $d$ & I & $d$ & $I$ \\
\hline \multirow[t]{2}{*}{$\begin{array}{lll}1 & 1 & 1 \\
2 & 0 & 0 \\
2 & 2 & 0\end{array}$} & $\begin{array}{l}2.777 \\
2.405 \\
1.700\end{array}$ & $\begin{array}{r}41 \\
100 \\
47\end{array}$ & $\begin{array}{l}2.878 \\
2.493 \\
1.7626\end{array}$ & $\begin{array}{r}71 \\
100 \\
5\end{array}$ & $\begin{array}{l}2.97 \\
2.58 \\
1.8243\end{array}$ & $\begin{array}{c}93 \\
100 \\
55\end{array}$ \\
\hline & \multicolumn{2}{|c|}{$\begin{array}{c}37-1497 \\
\mathrm{CaO}\end{array}$} & \multicolumn{2}{|c|}{$\begin{array}{c}23-127 \\
\mathrm{Ca}_{0.5} \mathrm{Sr}_{0.5} \mathrm{O}\end{array}$} & \multicolumn{2}{|c|}{$\begin{array}{c}6-520 \\
\mathrm{SrO}\end{array}$} \\
\hline hk $\ell$ & $d$ & I & $d$ & $I$ & d & 1 \\
\hline $\begin{array}{lll}1 & 1 & 1 \\
2 & 0 & 0 \\
2 & 2 & 0\end{array}$ & $\begin{array}{l}2.777 \\
2.405 \\
1.7009\end{array}$ & $\begin{array}{c}36 \\
100 \\
54\end{array}$ & $\begin{array}{l}2.853 \\
2.472 \\
1.7488\end{array}$ & $\begin{array}{r}90 \\
100 \\
80\end{array}$ & $\begin{array}{l}2.98 \\
2.581 \\
1.825\end{array}$ & $\begin{array}{r}90 \\
100 \\
80\end{array}$ \\
\hline $\begin{array}{l}\text { FOM } \\
\text { Nobs } \\
\text { Nposs }\end{array}$ & \multicolumn{2}{|c|}{$\begin{array}{c}116.08 \\
13 \\
13\end{array}$} & \multicolumn{2}{|c|}{$\begin{array}{l}17.06 \\
11 \\
12\end{array}$} & \multicolumn{2}{|c|}{$\begin{array}{l}82.89 \\
15 \\
15\end{array}$} \\
\hline$\Delta 2 \theta$ & \multicolumn{2}{|c|}{0.009} & \multicolumn{2}{|c|}{0.054} & \multicolumn{2}{|c|}{0.012} \\
\hline
\end{tabular}

Appendix $\mathrm{A}$ lists the simulated $2 \theta, \mathrm{d}, \mathrm{h} \mathrm{k} \ell$, and I values for the entire $(\mathrm{Ca}, \mathrm{Sr}) \mathrm{O}$ solid solution series. The (111) peak displays a $\Delta 2 \theta$ greater than $0.25^{\circ}$ as the $\mathrm{x}$ in $\mathrm{Ca}_{\mathrm{x}} \mathrm{Sr}_{1-\mathrm{x}} \mathrm{O}$ changes by 0.1 . The difference in $\Delta 2 \theta$ is larger yet for the other peaks in the patterns. Such differences are much larger than the typical acceptance window used for phase identification.

The PDF pattern for $\mathrm{CaO}$ (37-1497) agrees well with the simulated pattern and has an excellent FOM $[F(13)=116.08(0.009,13)]$. Note, however, that the peak at $d=0.80183$ should be attributed to the (412) reflection, not the (600).

The PDF pattern for SrO (6-520) does not compare as well to its calculated pattern as $\mathrm{CaO}$ did $[F(15)=82.89(0.0121,15)]$. Again, however, the peak at $d=0.8600$ should be attributed to the (442) reflection, not the (600). 
The PDF pattern for $\mathrm{Ca}_{0.5} \mathrm{Sr}_{0.5} \mathrm{O}(23-127)$ is not as accurate $[\mathrm{F}(11)=17.06$ $(0.054,12)]$ as the two cards for the two end members. Furthermore, the calculated density on the PDF card $\left(4.45 \mathrm{~g} / \mathrm{cm}^{3}\right)$ seems to be closer to that calculated for $\mathrm{Ca}_{0.4} \mathrm{Sr}_{0.6} \mathrm{O}$ $\left(4.441 \mathrm{~g} / \mathrm{cm}^{3}\right)$, not $\mathrm{Ca}_{0.5} \mathrm{Sr}_{0.5} \mathrm{O}\left(4.280 \mathrm{~g} / \mathrm{cm}^{3}\right)$. However, if the cell parameter is changed from $4.98529 \AA$ (the cell parameter used in the calculation assuming a linear change between the end member's cell parameters) to that of the PDF card ( $a=4.93 \AA$ ), the densities seem to agree. Consequently, determining which is more accurate, PDF 23-127 or the simulated pattern, requires more study.

Except for the end member patterns, it is recommended that all nine solid solution patterns be incorporated into the PDF.

\section{$3.2(\mathrm{Ca}, \mathrm{Sr})_{2} \mathrm{CuO}_{3}$}

Like $(\mathrm{Ca}, \mathrm{Sr}) \mathrm{O},(\mathrm{Ca}, \mathrm{Sr})_{2} \mathrm{CuO}_{3}$ has been shown to display a complete solid solution series across the phase diagram with a stability temperature up to $1030^{\circ} \mathrm{C}$ (refs. 2,3 ). Unfortunately, even though solid solution phases in this system have been reported, $4,6,7,16-25$ the only reference patterns available are for the end member phases 34-282 $\left(\mathrm{Ca}_{2} \mathrm{CuO}_{3}\right)$ [ref. 25] and 34-283 $\left(\mathrm{Sr}_{2} \mathrm{CuO}_{3}\right)$ [ref. 24]. Table 6 displays the crystallographic data for the end member phases. These data were used to simulate the solid solution series patterns. Again, a linear change in cell parameters was assumed.

Table 6. (Ca, $\mathrm{Sr})_{2} \mathrm{CuO}_{3}$ crystallographic parameters 22,23

\begin{tabular}{lccrccc}
\hline Struct. & S.G. & Atom & $\begin{array}{c}\text { Occ. } \\
0<x<1\end{array}$ & $\mathrm{x}$ & $\mathrm{y}$ & $\mathrm{z}$ \\
\hline Ortho & $\mathrm{Immm}$ & $\mathrm{Ca}$ & $4-4 \mathrm{x}$ & 0.15 & 0.0 & 0.5 \\
& $(71)$ & $\mathrm{Sr}$ & $4 \mathrm{x}$ & 0.15 & 0.0 & 0.5 \\
& & $\mathrm{Cu}$ & 2 & 0.0 & 0.5 & 0.0 \\
& & $\mathrm{O} 1$ & 4 & 0.34 & 0.0 & 0.5 \\
& & $\mathrm{O} 2$ & 2 & 0.0 & 0.0 & 0.0
\end{tabular}

This assumption is displayed in Table 7. The resulting simulated XRD data for the three strongest lines across the solid solution series are presented in Table 8 as well as the data for card 34-282 $\left(\mathrm{Ca}_{2} \mathrm{CuO}_{3}\right)$ and 34-283 $\left(\mathrm{Sr}_{2} \mathrm{CuO}_{3}\right)$. 
Table 7. (Ca,Sr) $)_{2} \mathrm{CuO}_{3}$ unit cell parameter (a), 24,25 $D_{\mathrm{x}}$, and $\mathrm{I} / \mathrm{I}_{\mathrm{c}}$

\begin{tabular}{cccccc}
\hline $\begin{array}{c}\text { Phase } \\
\text { Ca:Sr }\end{array}$ & $\mathrm{a}$ & $\mathrm{b}$ & $\mathrm{c}$ & $\mathrm{D}_{\mathrm{x}}$ & $\mathrm{I} / \mathrm{I}_{\mathrm{c}}$ \\
\hline & & & & & \\
\hline & & & & & \\
$2: 0$ & 12.2340 & 3.77690 & 3.25730 & 4.229 & 2.694 \\
$1.9: 0.1$ & 12.2565 & 3.78338 & 3.26922 & 4.303 & 2.775 \\
$1.8: 0.2$ & 12.2790 & 3.78985 & 3.28114 & 4.376 & 2.857 \\
$1.7: 0.3$ & 12.3015 & 3.79632 & 3.29306 & 4.447 & 2.939 \\
$1.6: 0.4$ & 12.3240 & 3.80280 & 3.30498 & 4.517 & 3.020 \\
$1.5: 0.5$ & 12.3465 & 3.80927 & 3.31690 & 4.586 & 3.102 \\
$1.4: 0.6$ & 12.3690 & 3.81575 & 3.32882 & 4.654 & 3.184 \\
$1.3: 0.7$ & 12.3915 & 3.22230 & 3.34074 & 4.721 & 3.265 \\
$1.2: 0.8$ & 12.4140 & 3.82870 & 3.35266 & 4.787 & 3.347 \\
$1.1: 0.9$ & 12.4365 & 3.83518 & 3.36458 & 4.852 & 3.429 \\
$1: 1$ & 12.4590 & 3.84165 & 3.37650 & 4.915 & 3.511 \\
$0.9: 1.1$ & 12.4815 & 3.84812 & $3.38: 542$ & 4.978 & 3.592 \\
$0.8: 1.2$ & 12.5040 & 3.85460 & 3.40034 & 5.040 & 3.674 \\
$0.7: 1.3$ & 12.5265 & 3.86107 & 3.41226 & 5.100 & 3.756 \\
$0.6: 1.4$ & 12.5490 & 3.86755 & 3.42418 & 5.160 & 3.838 \\
$0.5: 1.5$ & 12.5715 & 3.87403 & 3.43610 & 5.219 & 3.920 \\
$0.4: 1.6$ & 12.5940 & 3.88050 & 3.44802 & 5.276 & 4.002 \\
$0.3: 1.7$ & 12.6165 & 3.88698 & 3.44599 & 5.333 & 4.084 \\
$0.2: 1.8$ & 12.6615 & 3.89345 & 3.47186 & 5.379 & 4.159 \\
$0.1: 1.9$ & 12.6615 & 3.89992 & 3.48378 & 5.444 & 4.249 \\
$0: 2$ & 12.6840 & 3.90640 & 3.49570 & 5.498 & 4.317 \\
\hline & & & & &
\end{tabular}


Table 8. Three major lines of simulaied $\mathrm{Ca}_{2} \mathrm{CuO}_{3}$, $\mathrm{CaSrCuO}_{3}, \mathrm{Sr}_{2} \mathrm{CuO}_{3}$, and PDF cards: $34-282$ $\left(\mathrm{Ca}_{2} \mathrm{CuO}_{3}\right)$ and 34-283 $\left(\mathrm{Sr}_{2} \mathrm{CuO}_{3}\right)$

\begin{tabular}{|c|c|c|c|c|c|c|}
\hline \multirow[b]{2}{*}{$\underline{h k l}$} & \multicolumn{2}{|c|}{$\mathrm{Ca}_{2} \mathrm{CuO}_{3}$} & \multicolumn{2}{|c|}{$\mathrm{CaSrCuO}_{3}$} & \multicolumn{2}{|c|}{$\mathrm{Sr}_{2} \mathrm{CuO}_{3}$} \\
\hline & $d$ & $I$ & $d$ & $I$ & $d$ & 1 \\
\hline$\overline{310}$ & 2.771 & 55 & 2.820 & 63 & 2.869 & 68 \\
\hline 301 & 2.545 & 72 & 2.620 & 77 & 2.694 & 79 \\
\hline \multirow[t]{3}{*}{011} & 2.467 & 100 & 2.536 & 100 & 2.605 & 100 \\
\hline & \multirow{2}{*}{\multicolumn{2}{|c|}{$\begin{array}{c}34-282 \\
\mathrm{Ca}_{2} \mathrm{CuO}_{3}\end{array}$}} & & & \multirow{2}{*}{\multicolumn{2}{|c|}{$\begin{array}{c}34 \cdots 283 \\
\mathrm{Sr}_{2} \mathrm{CuO}_{3}\end{array}$}} \\
\hline & & & & & & \\
\hline$\underline{\mathrm{h}} \mathrm{k} \ell$ & & I & & & $d$ & I \\
\hline 310 & 2.771 & 55 & & & 2.870 & 65 \\
\hline 301 & 2.545 & 80 & & & 2.694 & 85 \\
\hline 011 & 2.467 & 100 & & & 2.604 & 100 \\
\hline FOM & 77. & & & & 81. & \\
\hline Nobs & 18 & & & & 16 & \\
\hline Nposs & 27 & & & & 24 & \\
\hline$\Delta 2 \theta$ & & & & & & \\
\hline
\end{tabular}

Appendix $\mathrm{B}$ lists the simulated $2 \theta, \mathrm{d}, \mathrm{h} \mathrm{k} \ell$, and I values for the entire $\mathrm{Ca}_{2-\mathrm{x}} \mathrm{Sr}_{\mathrm{x}} \mathrm{CuO}_{3}$ (for $x=0$ to 2 in steps of 0.1 ) solid solution series. The (011) peak displays a $\Delta 2 \theta$ greater than $0.2^{\circ}$ as $x$ changes by 0.2 .

The PDF cards available for the end members of this solid solution series $\left[\mathrm{Ca}_{2} \mathrm{CuO}_{3}\right.$ (34-282), $\mathrm{Sr}_{2} \mathrm{CuO}_{3}$ (34-283)] seem to agree well with the simulated patterns and have excellent FOMs, $F(18)=77.20(0.009,27)$ and $F(16)=81.23(0.008,24)$, respectively. Consequently, no change seems to be required. However, it is recommended that every other one of the solid solution patterns be incorporated into the PDF.

\section{$3.3(\mathrm{Ca}, \mathrm{Sr})_{14} \mathrm{Cu}_{24} \mathrm{O}_{41}$}

The structure, composition, and limit of Ca solubility in $\mathrm{Sr}_{14} \mathrm{Cu}_{24} \mathrm{O}_{41}$ has been debated by a number of authors. $3,4,6,7,26,27$ According to Siegrist et al., ${ }^{27} \mathrm{Sr}_{14} \mathrm{Cu}_{24} \mathrm{O}_{41}$ seems to display an incommensurate structure along the c-axis, but it becomes nearly commensurate at seven times the c-axis. McCarron et al. ${ }^{26}$ report that $\mathrm{Sr}_{14} \mathrm{Cu}_{24} \mathrm{O}_{41}$ is 
composed of two distinct subcells whose $\mathbf{a}$ and $\mathbf{b}$ parameters are equal but whose $\mathbf{c}$ parameters are $2.7 \AA$ for the $\mathrm{CuO}_{2}$ subcell and $3.9 \AA$ for the $\mathrm{Sr}_{2} \mathrm{Cu}_{2} \mathrm{O}_{3}$ subcell. These subcells become nearly commensurate at ten times the $2.7 \AA$ cell and seven times the $3.9 \AA$ cell.

The PDF contains two cards whose formulae are close to the same ratio of $\mathrm{Sr}: \mathrm{Cu}: \mathrm{O}$ and have cell parameters similar to the basic cell parameters described by Siegrist et al. ${ }^{27}$ These cards are 39-250 $\left(\mathrm{SrCu}_{2} \mathrm{O}_{3}\right)$ [ref. 28] and 39-489 $\left(\mathrm{Sr}_{1.75} \mathrm{Cu}_{3} \mathrm{O}_{5.13}\right)$ [ref. 29].

Table 9 displays the crystallographic data as described by Siegrist et al. ${ }^{27}$ Table 10 displays the cell parameters as a function of $\mathrm{Ca}$ concentration according to McCarron et al. 26 Table 11 displays the three major XRD lines of the reference patterns and simulated patterns across the solid solution series up to $\mathrm{Ca}_{7} \mathrm{~S}_{7} \mathrm{Cu}_{24} \mathrm{O}_{41}$. McCarron et al. ${ }^{26}$ reports that as the $\mathrm{Ca}$ concentration approaches $\mathrm{Ca}_{6} \mathrm{Sr}_{8} \mathrm{Cu}_{24} \mathrm{O}_{41}$, a structure change occurs. For this study this lower limit of $\mathrm{Ca}$ solubility was ignored.

Table 9. $(\mathrm{Ca}, \mathrm{Sr})_{14} \mathrm{Cu}_{24} \mathrm{O}_{41}$ crystallographic parameters ${ }^{27}$

\begin{tabular}{|c|c|c|c|c|c|c|}
\hline Struct. & S.G. & Atom & $\begin{array}{l}\text { Occ. } \\
0<x<1\end{array}$ & $x$ & $y$ & $\mathrm{z}$ \\
\hline \multirow[t]{16}{*}{ Ortho } & $\mathrm{Cccm}$ & $\mathrm{Sr}$ & $8-8 x$ & 0.2482 & 0.1168 & 0.0 \\
\hline & (66) & $\mathrm{Sr} 2$ & $16-16 x$ & 0.2514 & 0.1166 & (..14256 \\
\hline & & $\mathrm{Sr} 3$ & $16-16 x$ & 0.2489 & 0.1166 & 0.28566 \\
\hline & & Sr4 & $16-16 x$ & 0.2504 & 0.1166 & 0.42879 \\
\hline & & $\mathrm{Cu} 1$ & 16 & 0.0833 & 0.2492 & 0.07139 \\
\hline & & $\mathrm{Cu} 2$ & 16 & 0.0831 & 0.2549 & 0.21447 \\
\hline & & $\mathrm{Cu} 3$ & 16 & 0.0829 & 0.2452 & 0.35747 \\
\hline & & $\mathrm{Cu} 4$ & 8 & 0.0835 & 0.2544 & 0.500000 \\
\hline & & Cu5 & 4 & 0.0000 & 0.0000 & 0.000000 \\
\hline & & $\mathrm{Cu} 6$ & 8 & 0.0000 & 0.0000 & 0.10100 \\
\hline & & $\mathrm{Cu} 7$ & 8 & 0.0000 & 0.0000 & 0.19870 \\
\hline & & $\mathrm{Cu} 8$ & 8 & 0.0000 & 0.5000 & 0.04760 \\
\hline & & $\mathrm{Cu} 9$ & 8 & $0.0(0)(0)$ & $0.50(0)$ & $(0.14900)$ \\
\hline & & Cu10 & 4 & 0.00000 & $0.50(0)$ & $0.25(0)(0)$ \\
\hline & & 01 & 8 & 0.0864 & 0.2662 & $0.00(0) 00$ \\
\hline & & $\mathrm{O} 2$ & 16 & 0.0839 & 0.2404 & 0.14330 \\
\hline
\end{tabular}


Table 9. (continued)

\begin{tabular}{|c|c|c|c|c|c|c|}
\hline Struct. & S.G. & Atom & $\begin{array}{l}\text { Occ. } \\
0<x<1\end{array}$ & $x$ & $y$ & z \\
\hline & & $\mathrm{O} 3$ & 16 & 0.0834 & 0.2458 & 0.28660 \\
\hline & & O4 & 16 & 0.0851 & 0.2425 & 0.42880 \\
\hline & & O5 & 8 & 0.2500 & 0.2500 & 0.07060 \\
\hline & & 06 & 8 & 0.2500 & 0.2500 & 0.21470 \\
\hline & & 07 & 8 & 0.2500 & 0.2500 & 0.35780 \\
\hline & & $\mathrm{O} 8$ & 4 & 0.2500 & 0.2500 & 0.50000 \\
\hline & & O9 & 16 & 0.1181 & 0.0176 & 0.05350 \\
\hline & & $\mathrm{O} 10$ & 8 & 0.1184 & 0.0000 & 0.25000 \\
\hline & & O11 & 16 & 0.1211 & 0.0298 & 0.35160 \\
\hline & & $\mathrm{O} 12$ & 16 & 0.1196 & 0.4957 & 0.10370 \\
\hline & & $\mathrm{O} 13$ & 16 & 0.1203 & 0.4790 & 0.19650 \\
\hline & & O14 & 8 & 0.1208 & 0.4721 & 0.50000 \\
\hline & & Ca1 & $8 x$ & 0.2482 & 0.1168 & 0.00000 \\
\hline & & $\mathrm{Ca} 2$ & $16 x$ & 0.2514 & 0.1166 & 0.14256 \\
\hline & & $\mathrm{Ca} 3$ & $16 x$ & 0.0000 & 0.2489 & 0.11660 \\
\hline & & $\mathrm{Ca} 4$ & $16 x$ & 0.0000 & 0.2504 & 0.11660 \\
\hline
\end{tabular}

Table 10. $(\mathrm{Ca}, \mathrm{Sr}){ }_{14} \mathrm{Cu}_{24} \mathrm{O}_{41}$ unit cell parameters, ${ }^{26} \mathrm{D}_{\mathrm{x}}$, and $\mathrm{l} / \mathrm{I}_{\mathrm{C}}$

\begin{tabular}{lccccc}
\hline $\begin{array}{l}\text { Phase } \\
\text { Ca:Sr:Cu }\end{array}$ & a & $b$ & $c$ & $D_{x}$ & $\mathrm{I} / \mathrm{I}_{\mathrm{c}}$ \\
\hline & & & & & \\
\hline $0: 14: 24$ & 11.469 & 13.400 & 27.650 & 5.226 & 3.070 \\
$1: 13: 24$ & 11.448 & 13.354 & 27.659 & 5.282 & 2.989 \\
$2: 12: 24$ & 11.438 & 13.313 & 27.659 & 5.223 & 2.901 \\
$3: 11: 24$ & 11.420 & 13.257 & 27.610 & 5.187 & 2.820 \\
$4: 10: 24$ & 11.394 & 13.150 & 27.510 & 5.184 & 2.732 \\
$5: 9: 24$ & 11.380 & 13.060 & 27.450 & 5.160 & 2.644 \\
$6: 8: 24$ & 11.375 & 13.010 & 27.440 & 5.100 & 2.561 \\
$7: 7: 24$ & 11.371 & 12.986 & 27.392 & 5.049 & 2.489 \\
\hline
\end{tabular}


Table 11. Three major lines of simulated $\mathrm{Sr}_{14} \mathrm{Cu}_{24} \mathrm{O}_{41}, \mathrm{Ca}_{3} \mathrm{Sr}_{11} \mathrm{Cu}_{24} \mathrm{O}_{41}$, Ca $5 \mathrm{Sr}_{9} \mathrm{Cu}_{24} \mathrm{O}_{41}, \mathrm{Ca}_{7} \mathrm{Sr}_{7} \mathrm{Cu}_{24} \mathrm{O}_{41}$, observed $\mathrm{Sr}_{14} \mathrm{Cu}_{24} \mathrm{O}_{41}$ (ref. 4) and PDF cards $39-489\left(\mathrm{Sr}_{1.75} \mathrm{Cu}_{3} \mathrm{O}_{5.13}\right)$ and $39-250\left(\mathrm{SrCu}_{2} \mathrm{O}_{3}\right)$

\begin{tabular}{|c|c|c|c|c|c|c|c|}
\hline \multirow{5}{*}{$\begin{array}{l}\frac{\mathrm{hk} \ell}{240} \\
137 \\
317\end{array}$} & \multirow{2}{*}{$\frac{\mathrm{Sr}_{14} \mathrm{Cu}_{24} \mathrm{O}_{41}}{\mathrm{~d} \quad \mathrm{I}}$} & \multicolumn{2}{|c|}{$\mathrm{Ca}_{3} \mathrm{Sr}_{11} \mathrm{Cu}_{24} \mathrm{O}_{41}$} & \multicolumn{2}{|c|}{$\mathrm{Ca}_{5} \mathrm{Sr}_{9} \mathrm{Cu}_{24} \mathrm{O}_{41}$} & \multicolumn{2}{|c|}{$\mathrm{Ca}_{7} \mathrm{Sr}_{7} \mathrm{Cu}_{24} \mathrm{O}_{41}$} \\
\hline & & d & I & d & 1 & $d$ & I \\
\hline & $2.893 \quad 100$ & 2.866 & 100 & 2.832 & 93 & 2.813 & 100 \\
\hline & $2.865 \quad 85$ & 2.850 & 87 & 2.823 & 100 & 2.813 & 100 \\
\hline & $2.691 \quad 56$ & 2.682 & 54 & 2.669 & 46 & 2.665 & 41 \\
\hline & \multirow{2}{*}{$\begin{array}{c}39-250 \\
\mathrm{SrCu}_{2} \mathrm{O}_{3}\end{array}$} & \multirow{2}{*}{\multicolumn{2}{|c|}{$\begin{array}{l}\text { Roth et al. }{ }^{4} \\
\mathrm{Sr}_{14} \mathrm{Cu}_{24} \mathrm{O}_{41}\end{array}$}} & \multirow{2}{*}{\multicolumn{2}{|c|}{$\begin{array}{c}39.489 \\
\mathrm{Sr}_{1.75} \mathrm{Cu}_{3} \mathrm{O}_{5.13}\end{array}$}} & & \\
\hline & & & & & & & \\
\hline $\mathrm{hk \ell}$ & d & $d$ & $\mathrm{I}$ & d & $\mathrm{I}$ & & \\
\hline 240 & $2.898 \quad 100$ & 2.8879 & 100 & 2.894 & 100 & & \\
\hline 131 & 2.866 & 2.8608 & 66 & 2.867 & 80 & & \\
\hline 311 & 2.687 & 2.6853 & 52 & 2.692 & 42 & & \\
\hline FOM & 41.36 & 47.6 & & & & & \\
\hline bs & 29 & 30) & & 30 & & & \\
\hline Nposs & 56 & 32 & & 32 & & & \\
\hline$\Delta 2 \theta$ & 0.013 & & 17 & & 13 & & \\
\hline
\end{tabular}

Appendix $\mathrm{C}$ lists the complete $2 \theta, \mathrm{d}, \mathrm{h} \mathrm{k} \ell$, and $\mathrm{I}$ values for the entire $(\mathrm{Ca}, \mathrm{Sr})_{14} \mathrm{Cu}_{24} \mathrm{O}_{41}$ solid solution series from $\mathrm{Sr}_{14} \mathrm{Cu}_{24} \mathrm{O}_{41}$ up to $\mathrm{Ca}_{7} \mathrm{Sr}_{7} \mathrm{Cu}_{24} \mathrm{O}_{41}$. The (240) peak displays a $\Delta 2 \theta$ greater than $0.2^{\circ}$ as the $\mathrm{x}$ in $\mathrm{Ca}_{\mathrm{x}} \mathrm{Sr}_{14-\mathrm{x}} \mathrm{Cu}_{24} \mathrm{O}_{41}$ changes by 2. At $x=5$ the (240) peak overlaps the strong (137) and the apparent peak shifts significantly. It is therefore recommended that the patterns for $\mathrm{x}=0,2,4,5$, and 7 be incorporated into the PDF.

PDF card 39-489 ( $\mathrm{Sr}_{1.75} \mathrm{Cu}_{3} \mathrm{O}_{5.13}$ ) agrees fairly well with the simulated pattern for $\mathrm{Sr}_{14} \mathrm{Cu}_{24} \mathrm{O}_{41}$ in terms of $\mathrm{d}$ and $\mathrm{I}$. However, 39-489 reports a space group of Fmmm, whereas the simulated pattern (crystal structure taken from Siegrist et al. ${ }^{27}$ ) and the observed pattern reported by Roth et al. ${ }^{4}$ use a space group of $\mathrm{Cccm}$.

PDF card 39-250 with formula $\mathrm{SrCu}_{2} \mathrm{SrO}_{3}$ is likely $\mathrm{Sr}_{14} \mathrm{Cu}_{24} \mathrm{O}_{41}$, since $\mathrm{SrCu}_{2} \mathrm{O}_{3}$ does not occur in the phase diagram. This pattern seems to contain a number of extraneous peaks when compared to the simulated or the pattern observed by Roth et al. ${ }^{4}$ 
Furthermore, this pattern, although it has a "*" rating, does not show a space group or a calculated density.

The pattern observed by Roth et al. ${ }^{4}$ for $\mathrm{Sr}_{14} \mathrm{Cu}_{24} \mathrm{O}_{41}$ corresponds closely to the simulated pattern and has an adequate $\mathrm{FOM}[\mathrm{F}(30)=47.65(0.017,37)]$. We highly recommend that these simulated patterns and the pattern observed by Roth et al..$^{4}$ replace PDF cards 39-489 and 39-250.

\section{$3.4(\mathrm{Ca}, \mathrm{Sr}) \mathrm{CuO}_{2}$}

The $(\mathrm{Ca}, \mathrm{Sr}) \mathrm{CuO}_{2}$ solid solution series has been reported by a number of authors. $^{2-4,6,7,16,19,21,23,30-32}$ Most authors agree that the Ca solubility hits a limit around $\mathrm{Ca}_{0.75} \mathrm{Sr}_{0.25} \mathrm{CuO}_{2}$. If more $\mathrm{Ca}$ is added, a structure change from orthorhornbic to tetragonal occurs producing tetragonal $\mathrm{Ca}_{0,86} \mathrm{Sr}_{0.14} \mathrm{CuO}_{2}$ (refs. 2,6). At higher Ca concentrations, $\mathrm{CaCuO}_{2}$ forms with a different structure. $\mathrm{CaCuO}_{2}$ has been reported by Roth et $\mathrm{al}^{2}$ and is stable up to $740^{\circ} \mathrm{C}$. Orthorhombic $\mathrm{SrCuO}_{2}$ is stable up to $955^{\circ} \mathrm{C}$ (ref. 3).

Table 12 presents the crystallographic data for the $(\mathrm{Ca}, \mathrm{Sr}) \mathrm{CuO}_{2}$ solid solution series and the tetragonal phase. Table 13 displays the change in lattice parameters as a function of $\mathrm{Ca}$ concentration for the orthorhombic and tetragonal phases. Table 14 displays the PDF and simulated XRD data for the orthorhombic solid solution series. Table 15 displays the simulated and observed XRD data for the tetragonal phase.

Table 12. (Ca,Sr $)_{2} \mathrm{CuO}_{2}$ crystallographic parameters 23,30

\begin{tabular}{lccrccc}
\hline Struct. & S.G. & Atom & $\begin{array}{c}\text { Occ. } \\
\text { 0<x<i }\end{array}$ & x & $y$ & $z$ \\
\hline Ortho & $\mathrm{Cmcm}$ & $\mathrm{Sr}$ & $4-4 \mathrm{x}$ & 0.0000 & 0.3340 & 0.2500 \\
& $(63)$ & $\mathrm{Ca}$ & $4 \mathrm{x}$ & 0.0000 & 0.3340 & 0.2500 \\
& & $\mathrm{Cu}$ & 4 & 0.0000 & 0.0640 & 0.2500 \\
& & $\mathrm{O} 1$ & 4 & 0.0000 & 0.9450 & 0.2500 \\
Tetragonal & $\mathrm{P} 4 / \mathrm{mmm}$ & $\mathrm{O} 2$ & 4 & 0.0000 & 0.1780 & 0.2500 \\
$(0.14: 0.86)$ & $\mathrm{Sr}$ & $1-1 \mathrm{x}$ & 0.5000 & 0.5000 & 0.500() \\
& $(123)$ & $\mathrm{Ca}$ & $1 \mathrm{x}$ & 0.5000 & 0.5000 & 0.5000 \\
& & $\mathrm{Cu}$ & 1 & 0.0000 & 0.0000 & $0.00(0)$ \\
& & $\mathrm{O}$ & 2 & 0.0000 & 0.5000 & 0.0000 \\
\hline
\end{tabular}


Table 13. $(\mathrm{Ca}, \mathrm{Sr}) \mathrm{CuO}_{2}$ and $\mathrm{Ca} 0,86 \mathrm{Sr}\left(0,14 \mathrm{CuO}_{2}\right.$ unit cell parameters, ${ }^{2,23,31,32} \mathrm{D}_{\mathrm{x}}$, and $\mathrm{I} / \mathrm{I}_{\mathrm{c}}$

\begin{tabular}{lccccc}
\hline $\begin{array}{l}\text { Phase } \\
\text { Sr:Ca }\end{array}$ & a & $b$ & $c$ & $D_{x}$ & $1 / l_{c}$ \\
\hline & & & & & \\
\hline $1: 0$ & 3.5730 & 16.33130 & 3.91360 & 5.326 & 3.813 \\
$0.9: 0.1$ & 3.5492 & 16.291 .84 & 3.90548 & 5.246 & 3.639 \\
$0.8: 0.2$ & 3.5254 & 16.25238 & 3.89736 & 5.164 & 3.460 \\
$0.7: 0.3$ & 3.5016 & 16.21292 & 3.88924 & 5.080 & 2.294 \\
$0.6: 0.4$ & 3.4778 & 16.17346 & 3.88112 & 4.993 & 3.123 \\
$0.5: 0.5$ & 3.4540 & 16.13400 & 3.87300 & 4.904 & 2.953 \\
$0.4: 0.6$ & 3.4302 & 16.09454 & 3.86488 & 4.813 & 2.785 \\
$0.3: 0.7$ & 3.4064 & 16.05508 & 3.85676 & 4.719 & 2.618 \\
$0.25: 0.75$ & 3.3945 & 16.03535 & 3.85270 & 4.671 & 2.785 \\
$0.14: 0.86$ & 3.8611 & 3.8611 & 3.1995 & 4.952 & 5.455 \\
$1: 0$ & 10.588 & 2.8122 & 6.3245 & -- & -- \\
\hline
\end{tabular}

Table 14. Three main peaks of simulated $\mathrm{SrCuO}_{2}$,

$\mathrm{Ca}_{0.3} \mathrm{Sr}_{0.7} \mathrm{CuO}_{2}, \mathrm{Ca} 0.25 \mathrm{Sr}_{0.75} \mathrm{CuO}_{2}$, and

PDF cards: $39-1492\left(\mathrm{SrCuO}_{2}\right)$ and $38-1179\left(\mathrm{SrCuO}_{2}\right)$

\begin{tabular}{|c|c|c|c|c|c|c|}
\hline \multirow[b]{2}{*}{$\mathrm{hk} \mathrm{l}$} & \multicolumn{2}{|c|}{$\mathrm{SrCuO}_{2}$} & \multicolumn{2}{|c|}{$\mathrm{Cat}\left(0,3 \mathrm{Sr}\left(0,7 \mathrm{O}_{2}\right.\right.$} & \multicolumn{2}{|c|}{$\mathrm{Ca}() .75 \mathrm{Sr}_{(0.25} \mathrm{O}_{2}$} \\
\hline & $d$ & I & $d$ & $I$ & $d$ & $I$ \\
\hline 130 & 2.987 & 65 & 2.939 & 62 & 2.865 & 55 \\
\hline () 41 & 2.825 & 56 & 2.806 & 57 & 2.778 & 60 \\
\hline \multirow[t]{2}{*}{111} & 2.605 & 100 & 2.569 & 100 & 2.515 & 100 \\
\hline & \multicolumn{2}{|c|}{$\begin{array}{l}39-1492 \\
\mathrm{SrCuO}_{2}\end{array}$} & \multicolumn{2}{|c|}{$\begin{array}{l}38-1179 \\
\mathrm{SrCuO}_{2}\end{array}$} & & \\
\hline$\underline{h k l}$ & $d$ & I & $d$ & I & & \\
\hline 130 & 2.989 & 73 & 2.986 & 75 & & \\
\hline 041 & 2.826 & 71 & 2.825 & 75 & & \\
\hline 111 & 2.606 & 100 & 2.603 & 100 & & \\
\hline FOM & 75. & & 66 & & & \\
\hline Nobs & 30 & & 30 & & & \\
\hline Nposs & 35 & & 39 & & & \\
\hline$\Delta 2 \theta$ & & 010 & & 011 & & \\
\hline
\end{tabular}


Table 15. Four main peaks of tetragonal

$\mathrm{Ca} 0.86 \mathrm{Sr}_{0.14} \mathrm{CuO}_{2}$

\begin{tabular}{|c|c|c|}
\hline & Simulated & Roth et al, 2 \\
\hline $\mathrm{hk \ell}$ & d & $I$ \\
\hline 110 & 2.7300 & 2.7372 \\
\hline 101 & $2.4640 \quad 100$ & $2.4768 \quad 100$ \\
\hline 200 & 1.9305 & 1.9345 \\
\hline 211 & 1.5196 & 1.5254 \\
\hline
\end{tabular}

\begin{tabular}{ll} 
FOM & 50.76 \\
Nobs & 23 \\
Nposs & 23 \\
$\Delta 2 \theta$ & 0.013 \\
\hline
\end{tabular}

Appendix D lists the complete $2 \theta, \mathrm{d}, \mathrm{h} \mathrm{k} \ell$, and $\mathrm{I}$ values for the entire $(\mathrm{Ca}, \mathrm{Sr}) \mathrm{CuO}_{2}$ solid solution series up to $\mathrm{Ca}_{0.75} \mathrm{Sr}_{0.25} \mathrm{CuO}_{2}$. Appendix $\mathrm{E}$ lists the complete $\mathrm{XRD}$ data for the tetragonal phase $\left(\mathrm{Ca}_{0.86} \mathrm{Sr}_{0.14} \mathrm{CuO}_{2}\right)$. The (111) peak in the orthorhombic phase displays a $\Delta 2 \theta$ greater than $0.16^{\circ}$ as the $\mathrm{x}$ in $\mathrm{Ca}_{\mathrm{x}} \mathrm{Sr}_{1-\mathrm{x}} \mathrm{CuO}_{2}$ changes by 0.1 for the orthorhombic phase. It is therefore recommended that all of the orthorhombic patterns and the one tetragonal simulated pattern be incorporated into the PDF,

PDF cards 39-1492 (ref. 31) and 38-1179 (ref. 32) [both $\mathrm{SrCuO}_{2}$ ] agree reasonably well within the simulated patterns and have good FOMs $[\mathrm{F}(30)=75.95(0.010,39)$ and $F(30)=66.98(0.012,39)$, respectively]. However, since they are both dealing with the same phase, it is recommended that $38-1179$ be deleted as it has more discrepancies with the intensities of the simulated pattern.

Since no PDF card is available for the tetragonal phase, it is recommended that the pattern observed by Roth et al. ${ }^{2}[\mathrm{~F}(23)=50.76(0.013,23)]$ and the simulated pattern be incorporated into the PDF,

At the high-Ca end, the phase $\left(\mathrm{CaCuO}_{2}\right)$ has been reported by Roth et al. ${ }^{2}$ The four main peaks of the pattern for $\mathrm{CaCuO}_{2}$ observed by Roth et al, ${ }^{2}$ are presented in Table 16 . Available crystallographic data for this phase are limited, so a simulated pattern is not yet possible. However, a least-squares study of the pattern observed by Roth et al. ${ }^{2}$ indicates that it is relatively reliable $[F(15)=39.63(0.017,22)]$ and should be placed in the PDF until more data can be obtained. 
Table 16. Four main peaks for observed

$\mathrm{CaCuO}_{2}$ (ref, 2)

\begin{tabular}{llc}
\hline h kl & \multicolumn{1}{c}{$\mathrm{d}$} & $\mathrm{I}$ \\
\hline 002 & 3.1620 & 26 \\
202 & 2.7153 & 100 \\
311 & 2.0751 & 42 \\
511 & 1.6322 & 22 \\
FOM & 39.63 & \\
Nobs & 15 & \\
Nposs & 22 & \\
$\Delta 2 \theta$ & 0.0172 & \\
\hline
\end{tabular}

\section{5 $(\mathrm{Ca}, \mathrm{Sr}) \mathrm{Cu}_{2} \mathrm{O}_{3}$}

The existence of $\mathrm{CaCu}_{2} \mathrm{O}_{3}$ has been reported by many authors. ${ }^{2,6,20,33-35}$ The atomic positions and crystal structure of four different models as compiled by Teske et al. ${ }^{34}$ and Hoppe ${ }^{35}$ are present in Table 17. Table 17 also contains the $\mathrm{I} / \mathrm{I}_{\mathrm{c}}$ and $\mathrm{D}_{\mathrm{x}}$ values calculated in our studies. According to Roth et al., ${ }^{2} \mathrm{CaCu}_{2} \mathrm{O}_{3}$ is only stable from 920 to $1\left(020^{\circ} \mathrm{C}\right.$.

Table 17. $(\mathrm{Ca}, \mathrm{Sr}) \mathrm{Cu}_{2} \mathrm{O}_{3}$ crystallographic parameters 35 and $\mathrm{I} / \mathrm{I}_{\mathrm{c}}$ (for all four models: $\mathrm{a}=9.942 ; \mathrm{b}=4.0786$;

$$
\left.c=3.459 ; D_{x}=5.094\right)
$$

\begin{tabular}{|c|c|c|c|c|c|c|c|}
\hline $\begin{array}{l}\text { Model/ } \\
\text { structure }\end{array}$ & S.G. & Atom & $\begin{array}{l}\text { Occ. } \\
0<x<1\end{array}$ & $x$ & $y$ & $z$ & $\mathrm{I} / \mathrm{I}_{\mathrm{C}}$ \\
\hline \multirow[t]{4}{*}{ 1/Ortho } & \multirow[t]{4}{*}{$\underset{(59)}{P \text { Pmma }}$} & $\begin{array}{l}\mathrm{Ca} \\
\mathrm{Cu}\end{array}$ & $\begin{array}{c}2-2 x \\
4\end{array}$ & $\begin{array}{l}0.25 \\
0.084\end{array}$ & $\begin{array}{l}0.25 \\
0.75\end{array}$ & $\begin{array}{l}0.3633 \\
0.84\end{array}$ & \multirow[t]{4}{*}{1.686} \\
\hline & & $O(1)$ & 2 & 0.25 & 0.75 & 0.595 & \\
\hline & & $O(2)$ & 4 & 0.079 & 0.25 & 0.871 & \\
\hline & & $\mathrm{Sr}$ & $2 x$ & 0.25 & 0.25 & 0.3633 & \\
\hline \multirow[t]{5}{*}{ 2/Ortho } & \multirow{5}{*}{$\underset{(59)}{\text { Pmma }}$} & $\mathrm{Ca}$ & $2-2 x$ & 0.25 & 0.25 & 0.3633 & \multirow[t]{5}{*}{1.742} \\
\hline & & $\mathrm{Cu}$ & 4 & 0.081 & 0.75 & 0.847 & \\
\hline & & $\mathrm{O}(1)$ & 2 & 0.25 & 0.75 & 0.595 & \\
\hline & & $O(2)$ & 4 & 0.087 & 0.25 & 0.826 & \\
\hline & & $\mathrm{Sr}$ & $2 x$ & 0.25 & 0.25 & 0.3633 & \\
\hline
\end{tabular}


Table 17. (contunued)

\begin{tabular}{lccrcccc}
\hline $\begin{array}{l}\text { Model/ } \\
\text { structure }\end{array}$ & S.G. & Atom & $\begin{array}{l}\text { Occ. } \\
0<\mathrm{x}<1\end{array}$ & $\mathrm{x}$ & $\mathrm{y}$ & $\mathrm{z}$ & $\mathrm{I} / \mathrm{I}_{\mathrm{c}}$ \\
\hline 3/Ortho & $\mathrm{Pmma}$ & $\mathrm{Ca}$ & $2-2 \mathrm{x}$ & 0.25 & 0.25 & 0.3633 & 2.302 \\
& $(59)$ & $\mathrm{Cu}$ & 4 & 0.084 & 0.75 & 0.84 & \\
& & $\mathrm{O}(1)$ & 2 & 0.25 & 0.75 & 0.115 & \\
4/Ortho & & $\mathrm{O}(2)$ & 4 & 0.088 & 0.25 & 0.833 & \\
& $\mathrm{Srmma}$ & $\mathrm{Ca}$ & $2 \mathrm{x}$ & 0.25 & 0.25 & 0.3633 & \\
& $\mathrm{C}$ & $\mathrm{Ca}$ & 0.25 & 0.25 & 0.3633 & 2.244 \\
& & $\mathrm{Cu}$ & 4 & 0.084 & 0.75 & 0.84 & \\
& & $\mathrm{O}(1)$ & 2 & 0.250 & 0.75 & 0.115 & \\
& & $\mathrm{O}(2)$ & 4 & 0.082 & 0.25 & 0.853 & \\
& $\mathrm{Sr}$ & $2 \mathrm{x}$ & 0.25 & 0.25 & 0.3633 & \\
\hline
\end{tabular}

More data are necessary to determine the limit of solubility of $\mathrm{Sr}$ in this phase.

Table 18 presents the simulated patterns of the four different models of $\mathrm{CaCu}_{2} \mathrm{O}_{3}$ (ref. 33).

To determine which model is most accurate, more experimental data are needed.

Table 18. Three strongest lines for the four clifferent structure models of $\mathrm{CaCu}_{2} \mathrm{O}_{3}$ presented by $\mathrm{Hoppe}^{35}$ and PDF card 34-284 $\left(\mathrm{CaCu}_{2} \mathrm{O}_{3}\right)$

\begin{tabular}{|c|c|c|c|c|c|c|}
\hline $\mathrm{hkl}$ & d & $\frac{\text { Model: } 1}{I}$ & $\frac{2}{I}$ & $\frac{3}{I}$ & $\frac{4}{I}$ & $\frac{34-284}{\mathrm{~d}}$ \\
\hline $\begin{array}{llll}0 & 1 & 1 \\
3 & 1 & 0 \\
3 & 0 & 1\end{array}$ & $\begin{array}{l}2.638 \\
2.572 \\
2.393\end{array}$ & $\begin{array}{c}91 \\
74 \\
100\end{array}$ & $\begin{array}{c}75 \\
72 \\
100\end{array}$ & $\begin{array}{r}40 \\
55 \\
100\end{array}$ & $\begin{array}{r}44 \\
56 \\
100\end{array}$ & $\begin{array}{rr}2.639 & 100 \\
2.571 & 60 \\
2.392 & 70\end{array}$ \\
\hline $\begin{array}{l}\text { FOM } \\
\text { Nobs } \\
\text { Npos }\end{array}$ & & & & & & $\begin{array}{l}65.43 \\
20 \\
32\end{array}$ \\
\hline$\Delta 2 \theta$ & & & & & & 0.010 \\
\hline
\end{tabular}

Appendix $\mathrm{F}$ lists the complete $2 \theta, \mathrm{d}, \mathrm{h} \mathrm{k} \ell$, and I values for all four models of $\mathrm{CaCu}_{2} \mathrm{O}_{3}$. The intensities change significantly, 
The simulated patterns for $\mathrm{CaCu}_{2} \mathrm{O}_{3}$ seem to differ significantly from the PDF card $34-284[F(20)=65.43(0.0010,32)]$. Experimental studies on this particular phase and the effects of Sr substitution are currently under way.

\section{$3.6(\mathrm{Ca}, \mathrm{Sr}) \mathrm{Cu}_{2} \mathrm{O}_{2}$}

The effects of $\mathrm{Ca}$ solid solution on $\mathrm{SrCu}_{2} \mathrm{O}_{2}$ have not been studied, and as a result, powder pattern simulations cannot be calculated. However, Teske et al. ${ }^{36}$ and Klockow et $a 1^{37}$ have extensively studied the known end member of $\mathrm{SrCu}_{2} \mathrm{O}_{2}$, which can only be produced in in $\mathrm{O}_{2}$-deficient atmosphere. Since such conditions are common in the formation of BSCCO superconductors, this phase should be more closely studied. Tables 19, 2(), and 21 display the crystallographic data, lattice parameters, the three strongest lines of PDF card 38-1178 $\left(\mathrm{SrCu}_{2} \mathrm{O}_{2}\right)$ [ref. 37] and a simulated pattern for this phase, respectively.

Table 19. $\mathrm{SrCu}_{2} \mathrm{O}_{2}$ crystallographic parameters ${ }^{36}$

\begin{tabular}{lcccccl}
\hline Structure & S.G. & Atom & Occ. & $x$ & $y$ & \multicolumn{1}{c}{$z$} \\
\hline \multirow{2}{*}{ Tet. } & \multirow{2}{*}{141} & $\mathrm{Sr}$ & 4 & 0.0 & 0.0 & 0.0 \\
& & $\mathrm{Cu}$ & 8 & 0.0 & 0.25 & 0.625 \\
& & $\mathrm{O}$ & 8 & 0.0 & 0.0 & 0.5 \\
\hline
\end{tabular}

Table 20. $\mathrm{SrCu}_{2} \mathrm{O}_{2}$ unit cell parameters, ${ }^{37} \mathrm{D}_{\mathrm{x}}$, and $\mathrm{I} / \mathrm{I}_{\mathrm{C}}$

\begin{tabular}{lccccc}
\hline Phase & $a$ & $b$ & $c$ & $D_{x}$ & $1 / I_{c}$ \\
\hline $\mathrm{SrCu}_{2} \mathrm{O}_{2}$ & 5.469 & 5.469 & 9.826 & 5.575 & 4.384 \\
\hline
\end{tabular}


Table 21. Three main peaks of $\mathrm{SrCu}_{2} \mathrm{O}_{2}$ and PDF card 38-1178 $\left(\mathrm{SrCu}_{2} \mathrm{O}_{2}\right)$

\begin{tabular}{|c|c|c|c|c|}
\hline \multirow[b]{2}{*}{$\mathrm{h} \mathrm{kl}$} & \multicolumn{2}{|c|}{$\mathrm{SrCu}_{2} \mathrm{O}_{2}$} & \multicolumn{2}{|c|}{$38-1178$} \\
\hline & $\mathrm{d}$ & I & $\mathrm{d}$ & I \\
\hline $\begin{array}{lll}1 & 1 & 2 \\
1 & 0 & 3 \\
2 & 1 & 1\end{array}$ & $\begin{array}{l}3.039 \\
2.810 \\
2.373\end{array}$ & $\begin{array}{c}100 \\
51 \\
66\end{array}$ & $\begin{array}{l}3.041 \\
2.811 \\
2.372\end{array}$ & $\begin{array}{r}20 \\
90 \\
100\end{array}$ \\
\hline $\begin{array}{l}\text { FOM } \\
\text { Nobs } \\
\text { Npos }\end{array}$ & & & $\begin{array}{l}37.0 \\
25 \\
49\end{array}$ & \\
\hline$\Delta 2 \theta$ & & & 0.1 & \\
\hline
\end{tabular}

Appendix $\mathrm{G}$ lists the complete $2 \theta, \mathrm{d}, \mathrm{h} \mathrm{k} \ell$, and I values for $\mathrm{SrCu}_{2} \mathrm{O}_{2}$. Though the $2 \theta$ and $h \mathrm{k} \ell$ values for this simulated pattern and PDF card 38-1178 $[F(25)=37.01(0.014,49)]$ are in reasonable agreement, the intensities differ significantly. Consequently, more data are needed to determine which pattern is correct. Until the ambiguity is resolved, both patterns should be in the PDF.

\section{CONCLUSIONS}

The XRD patterns of phases found in the $\mathrm{CaO}-\mathrm{SrO}-\mathrm{CuO}$ ternary system have been reviewed and simulated to produce recommended powder patterns for these phases. In order to obtain a complete understanding of the BSCCO system, a complete XRD study of the $\mathrm{Ca}-\mathrm{Sr}-\mathrm{Cu}-\mathrm{O}$ phases must include further analysis of the $(\mathrm{Ca}, \mathrm{Sr}) \mathrm{Cu}_{2} \mathrm{O}_{3}$, and the $(\mathrm{Ca}, \mathrm{Sr}) \mathrm{Cu}_{2} \mathrm{O}_{2}$ systems. Without this analysis, phases formed in low-oxygen partial pressures or that are only present at high temperatures cannot be accurately identified.

\section{ACKNOWLEDGMENTS}

The assistance of the International Centre for Diffraction Data, O. B. Cavin of the High Temperature Materials Laboratory X-ray Diffraction User Center, the support of the Oak Ridge Science and Engineering Research Semester, and Oak Ridge Associated Universities are gratefully acknowledged. 


\section{REFERENCES}

1. H. Maeda et al., "A New High-Tc Oxide Superconductor without a Rare Earth Element," Jpn. J. Appl. Phys. 27, L209 (1988).

2. R. Roth et al., "Phase Equilibria of the System SrO-CaO-CuO," J. Am. Ceram. Soc. 72(8), 1545 (1989).

3. R. Roth et al., "Phase Equilibria and Crystal Chemistry in the Quaternary System Ba-Sr-Y-Cu-O in Air," J. Am. Ceram. Soc. 72(3), 395 (1989).

4. R. Roth et al., "Phase Equilibria and Crystal Chemistry in Portions of the System

SrO-CaO- $\mathrm{Bi}_{2} \mathrm{O}_{3}-\mathrm{CuO}$, Part II - The System SrO- $\mathrm{Bi}_{2} \mathrm{O}_{3}-\mathrm{CuO}, "$ J. Res. Natl. Inst. Stand. Technol. 95, 291 (1990).

5. P. Majewski et al., "Phase Equilibrium Diagrams of $\mathrm{Bi}_{2} \mathrm{O}_{3}$-SrO-CaO-CuO," to be published in Adv. Mater.

6. K. Schultze et al., "Phase Equilibrium in the System $\mathrm{Bi}_{2} \mathrm{O}_{3}$-SrO-CaO-CuO - A Tool for Processing the High-Tc Superconducting Bismuth Compounds," to be published in Zeitschrift fur Metallkunde.

7. M. Vallino et al., "Subsolidus Phase Relations in the SrO-CaO-CuO-O and SrO-Y2O3-CaO-O Systems," Mater. Chem. Phys. 22, 523 (1989).

8. C. Lee et al., "Equilibrium Phase Relations in the Bi-Ca-Sr-Cu-O system at $850 \& 900)^{\circ} \mathrm{C}, "$ J. Mater. Res. 5(7), 1403 (1990).

9. D. Matheis and R. Snyder, "Crystal Structure and Powder Diffraction Patterns of the Bismuth and Thallium Ruddlesdon Popper Copper Oxide Superconductors," Powder Diff. 5(1), 8 (1990).

10. D. Smith, and K. Smith, "MICRO-POWD: A Program for Calculating X-ray Powder Diffraction Patterns on a P.C.," Materials Data, Inc., Copyright 1987.

11. H. Evens, Jr, D. Appleman, and D. Handwerder, Report PB216188, U.S. Dept. of Commerce, National Technical Information Center, Springfield, Va., 1963.

12. H. McMurdie et al., "Standard X-ray Diffraction Powder Patterns from the JCPDS Research Associateship," Powder Diff. 1(3), 265 (1986).

13. H. Swanson, N. Gilfrich, and G. Ugrinic, "Standard X-ray Diffraction Powder Patterns," Natl. Bur. Stand. (U.S.) Circ. 529 5, 69 (1955).

14. Obst and Munchberg, Tonid. Zeitg. 6, 92 (1968).

15. H. Swanson et al., "Standard X-ray Diffraction Powder Patterns," Natl. Bur. Stand. Monog. 25(5), 43 (1967). 
16. W. Carrilo-CaLrera, and W. Göpel, " Influence of High-Temperature Annealing on the $(\mathrm{Bi}, \mathrm{Pb})_{2} \mathrm{Sr}_{2} \mathrm{Ca}_{2} \mathrm{Cu}_{3} \mathrm{O}_{10}$ Phase and Determination of its Crystal Structure by $\mathrm{X}$-ray Powder Diffractometry," Phys. C 161, 373 (1989).

17. H. Hoshizaki et al., "Direct Observations of Melting and Solidification of Bi-Pb-Sr-Ca-Cu-O Compounds," Jpn. J. App. Phys. 29, 1444 (1990).

18. H. Susakura et al., "Single High-Tc Phase Regions of the Bi-Pb-Sr-Ca-Cu-O System," Jpn. J. App. Phys. 28(7), L1163 (1989).

19. Y. Oka et al., "Crystalline Phases formed in the Partially Melted States of Bi-Ca-Sr-Cu-O," Jpn. J. Appl. Phys. 28(5), L801 (1989).

20. P. Trembley and M. Gasgnier, "Energy Dispersive X-ray (EDX) Chemical Analysis of High-Tx Superconducting Phases in the Bi-Ca-Sr-Cu-O System," J. Microsc. Spectrosc. Electron. 14, 75 (1989).

21. H. Muller-Buschbaum, "Oxometallates w/Planar Coordination," Angew. Chem. Int. Ed. Eng. 16, 674 (1987).

22. V.C. L. Teske and H. Muler-Buschbaum, "Zur Kennitnis von $\mathrm{Sr}_{2} \mathrm{CuO}_{3}$," Zeit. Anorg. Allge. Chemie. 371, 325 (1969).

23. V.C. L. Teske and H. Muler-Buschbaum, "Zur Kennitnis von $\mathrm{Ca}_{2} \mathrm{CuO}_{3}$ und $\mathrm{SrCuO}_{2}$," Zeit. Anorg. Allge. Chemie. 379, 234 (1970).

24. Breuer and Eysel, JCPDS Grant-in-Aid Report, 1982.

25. Breuer and Eysel, JCPDS Grant-in-Aid Report, 1981.

26. E. McCarron III et al., "The Incommensurate Structure of $\left(\mathrm{Sr}_{14-x} \mathrm{Ca}_{x}\right) \mathrm{Cu}_{24} \mathrm{O}_{41}$ $(0<x \sim 8)$ A Superconductor By-product," Maier. Res, Bull. 23, 1355 (1988).

27. T. Siegrist et al., "A New Layered Cuprate Structure Type $\left(\mathrm{A}_{1-\mathrm{x}} \mathrm{A}_{\mathrm{x}}^{\prime}\right)_{14} \mathrm{Cu}_{24} \mathrm{O}_{41}$," Mater. Res. Bull. 23. 1429 (1988).

28. Klockow and Eysel, JCPDS Grant-in-Aid Report, 1988.

29. W. Wong-Ng et al., JCPDS Grant-in-Aid Report 1988.

30. T. Siegrist et al., "The Parent Structure of the Layered High-Temperature Superconductor," Nature 334, 21 (1988).

31. W. Wong-Ng et al., JCPDS Grant-in-Aid Report, 1987.

32. Popp and Eysel, JCPDS Grant-in-Aid Report, 1987.

33. Breuer and Eysel, JCPDS Grant-in-Aid Report, 1982.

34. V.C. L. Teske and H. Muller-Buschbaum, "Zur Kennitnis von $\mathrm{CaCu}_{2} \mathrm{O}_{3}$," Zeit. Anorg. Allge. Chemie. 370, 134 (1969).

35. V. R. Hoppe, "Zur Gitterenergie von $\mathrm{CaCu}_{2} \mathrm{O}_{3}$," Zeit. Anorg. Allge. Chemie. 370, 144 (1969). 
36. V.C. L. Teske and H. Muller-Buschbaum, "Zur Kennitnis von $\mathrm{SrCu}_{2} \mathrm{O}_{2}$," Zeit. Anorg. Allge. Chemie. 379, 113 (1970).

37. Klockow and Eysel, JCPDS Grant-in-Aid Report, 1987. 
APPENDIX A: $(\mathrm{Ca}, \mathrm{Sr}) \mathrm{O}$ 
Table A.1, Simulated X-ray powder pattern for $\mathrm{CaO}$

\begin{tabular}{rrllllllrr}
\hline $2 \theta$ & PEAK & $d$ & $h$ & \multicolumn{3}{c}{$l$} & $l(I N T) I(P K) I(D S)$ \\
\hline 32.20 & 32.20 & 2.777 & 1 & 1 & 1 & 40 & 41 & 33 \\
37.36 & 37.36 & 2.405 & 2 & 0 & 0 & 100 & 100 & 100 \\
53.86 & 53.86 & 1.7008 & 2 & 2 & 0 & 51 & 47 & 84 \\
64.16 & 64.16 & 1.4504 & 3 & 1 & 1 & 17 & 15 & 36 \\
67.38 & 67.38 & 1.3887 & 2 & 2 & 2 & 14 & 12 & 31 \\
& & & & & & & & \\
79.66 & 79.66 & 1.2026 & 4 & 0 & 0 & 5 & 5 & 16 \\
88.53 & 88.52 & 1.1036 & 3 & 3 & 1 & 7 & 5 & 23 \\
91.47 & 91.46 & 1.0757 & 4 & 2 & 0 & 14 & 11 & 50 \\
103.34 & 103.34 & .9820 & 4 & 2 & 2 & 11 & 9 & 47 \\
112.62 & 112.62 & .9258 & 5 & 1 & 1 & 5 & 5 & 25 \\
& & & & & & & & \\
112.62 & & .9258 & 3 & 3 & 3 & 2 & & 8 \\
129.86 & 129.86 & .8504 & 4 & 4 & 0 & 5 & 3 & 31 \\
142.64 & 142.64 & .8131 & 5 & 3 & 1 & 15 & 7 & 97 \\
147.79 & 147.78 & .8018 & 4 & 4 & 2 & 15 & 7 & 101 \\
147.79 & & .8018 & 6 & 0 & 0 & 4 & & 25 \\
\hline
\end{tabular}

Table A.2. Simulated X-ray powder pattem for $\mathrm{Ca}_{0.9} \mathrm{Sr}_{0.1} \mathrm{O}$

\begin{tabular}{rrlllllrrr}
\hline $2 \theta$ & PEAK & $d$ & $h$ & \multicolumn{4}{c}{$l($ INT)I(PK)I(DS) } \\
\hline 31.97 & 31.98 & 2.798 & 1 & 1 & 1 & 47 & 48 & 39 \\
37.08 & 37.08 & 2.423 & 2 & 0 & 0 & 100 & 100 & 100 \\
53.44 & 53.44 & 1.7132 & 2 & 2 & 0 & 52 & 48 & 87 \\
63.64 & 63.64 & 1.4610 & 3 & 1 & 1 & 20 & 17 & 42 \\
66.83 & 66.84 & 1.3988 & 2 & 2 & 2 & 14 & 12 & 33 \\
& & & & & & & & \\
78.97 & 78.98 & 1.2114 & 4 & 0 & 0 & 6 & 5 & 17 \\
87.73 & 87.72 & 1.1116 & 3 & 3 & 1 & 8 & 6 & 27 \\
90.62 & 90.62 & 1.0835 & 4 & 2 & 0 & 15 & 12 & 54 \\
102.30 & 102.30 & .9891 & 4 & 2 & 2 & 12 & 9 & 49 \\
111.39 & 111.38 & .9325 & 5 & 1 & 1 & 6 & 6 & 28 \\
& & & & & & & & \\
111.39 & & .9325 & 3 & 3 & 3 & 2 & & 9 \\
128.13 & 128.12 & .8566 & 4 & 4 & 0 & 6 & 3 & 32 \\
140.27 & 140.26 & .8190 & 5 & 3 & 1 & 16 & 7 & 103 \\
145.04 & 145.04 & .8076 & 4 & 4 & 2 & 15 & 8 & 98 \\
145.04 & & .8076 & 6 & 0 & 0 & 4 & & 25 \\
\hline
\end{tabular}


Table A.3. Simulated X-ray powder pattern for $\mathrm{Ca}_{0.8} \mathrm{Sr}_{0.2} \mathrm{O}$

\begin{tabular}{|c|c|c|c|c|c|c|c|c|}
\hline $2 \theta$ & PEAK & d & $h$ & k & 0111 & NT)! & (PK) & $\mid(D S)$ \\
\hline 31.73 & 31.74 & 2.818 & 1 & 1 & 1 & 53 & 55 & 44 \\
\hline 36.80 & 36.80 & 2.440 & 2 & 0 & 01 & 00 & 100 & 100 \\
\hline 53.03 & 53.04 & 1.7255 & 2 & 2 & 0 & 53 & 48 & 89 \\
\hline 63.13 & 63.14 & 1.4715 & 3 & 1 & 1 & 22 & 20 & 48 \\
\hline 66.29 & 66.28 & 1.4089 & 2 & 2 & 2 & 15 & 13 & 34 \\
\hline 78.30 & 78.30 & 1.2201 & 4 & 0 & 0 & 6 & 5 & 18 \\
\hline 86.94 & 86.94 & 1.1197 & 3 & 3 & 1 & 9 & 7 & 30 \\
\hline 89.80 & 89.80 & 1.0913 & 4 & 2 & 0 & 16 & 13 & 57 \\
\hline 101.29 & 101.28 & 9962 & 4 & 2 & 2 & 12 & 9 & 52 \\
\hline 110.19 & 110.20 & 9392 & 5 & 1 & 1 & 6 & 6 & 31 \\
\hline 110.19 & & .9392 & 3 & 3 & 3 & 2 & & 10 \\
\hline 126.46 & 126.46 & .8628 & 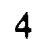 & 4 & 0 & 6 & 3 & 33 \\
\hline 138.06 & 138.06 & .8249 & 5 & 3 & 1 & 16 & & 107 \\
\hline 142.52 & 142.52 & .8134 & 4 & 4 & 2 & 14 & 8 & 96 \\
\hline 142.52 & & .8134 & 6 & 0 & 0 & 4 & & 24 \\
\hline
\end{tabular}

Table A.4. Simulated X-ray powder pattem for $\mathrm{Ca}_{0.7} \mathrm{Sr}_{0.3 \mathrm{O}}$

\begin{tabular}{rrlllllrrr}
\hline $2 \theta$ & PEAK & $d$ & $h$ & $k$ & $l($ INT)|(PK)I(DS) \\
\hline 31.50 & 31.50 & 2.838 & 1 & 1 & 1 & 59 & 61 & 49 \\
36.53 & 36.54 & 2.458 & 2 & 0 & 0 & 100 & 100 & 100 \\
52.62 & 52.62 & 1.7379 & 2 & 2 & 0 & 55 & 50 & 92 \\
62.63 & 62.64 & 1.4821 & 3 & 1 & 1 & 25 & 22 & 54 \\
65.76 & 65.76 & 1.4190 & 2 & 2 & 2 & 15 & 13 & 36 \\
77.64 & 77.64 & 1.2289 & 4 & 0 & 0 & 6 & 5 & 19 \\
86.17 & 86.18 & 1.1277 & 3 & 3 & 1 & 10 & 8 & 33 \\
88.99 & 88.98 & 1.0991 & 4 & 2 & 0 & 16 & 13 & 60 \\
100.30 & 100.30 & 1.0034 & 4 & 2 & 2 & 12 & 10 & 54 \\
109.04 & 109.04 & .9460 & 5 & 1 & 1 & 7 & 7 & 33 \\
109.04 & & & .9460 & 3 & 3 & 3 & 2 & & 11 \\
109.04 & 124.88 & .8689 & 4 & 4 & 0 & 6 & 4 & 33 \\
135.98 & 135.98 & .8309 & 5 & 3 & 1 & 17 & 8 & 110 \\
140.19 & 140.20 & .8192 & 4 & 4 & 2 & 14 & 8 & 94 \\
140.19 & & .8192 & 6 & 0 & 0 & 3 & & 24 \\
\hline
\end{tabular}


Table A.5. Simulated X-ray powder pattern for

\begin{tabular}{rrrrrrrrrr}
\multicolumn{10}{c}{$\mathrm{Ca}_{0.6} \mathrm{Sr}_{0.4} \mathrm{O}$} \\
\hline $2 \theta$ & PEAK & $d$ & $\mathrm{~d}$ & $k$ & $l$ & \\
\hline 31.27 & 31.28 & 2.858 & 1 & 1 & 1 & 64 & 67 & 53 \\
36.26 & 36.26 & 2.475 & 2 & 0 & 0 & 100 & 100 & 100 \\
52.22 & 52.22 & 1.7502 & 2 & 2 & 0 & 56 & 51 & 94 \\
62.14 & 62.14 & 1.4926 & 3 & 1 & 1 & 27 & 24 & 59 \\
65.24 & 65.24 & 1.4290 & 2 & 2 & 2 & 16 & 14 & 37 \\
& & & & & & & & \\
76.99 & 76.98 & 1.2376 & 4 & 0 & 0 & 6 & 5 & 19 \\
85.42 & 85.42 & 1.1357 & 3 & 3 & 1 & 10 & 9 & 37 \\
88.20 & 88.20 & 1.1069 & 4 & 2 & 0 & 17 & 14 & 62 \\
99.34 & 99.34 & 1.0105 & 4 & 2 & 2 & 13 & 10 & 56 \\
107.91 & 107.90 & .9527 & 5 & 1 & 1 & 7 & 7 & 36 \\
& & & & & & & & \\
107.91 & & .9527 & 3 & 3 & 3 & 2 & & 12 \\
123.34 & 123.34 & .8751 & 4 & 4 & 0 & 6 & 4 & 34 \\
134.02 & 134.02 & .8368 & 5 & 3 & 1 & 17 & 9 & 113 \\
138.02 & 138.02 & .8251 & 4 & 4 & 2 & 13 & 8 & 93 \\
138.02 & & .8251 & 6 & 0 & 0 & 3 & & 23 \\
\hline
\end{tabular}

Table A.6. Simulated X-ray powder pattern for

$$
\mathrm{Ca}_{0.5} \mathrm{Sr}_{0.5^{\mathrm{O}}}
$$

\begin{tabular}{rrrrrrrrrr}
\hline $2 \theta$ & PEAK & $d$ & $h$ & $k$ & \multicolumn{3}{l}{$l($ INT)I(PK)I(DS) } \\
\hline 31.05 & 31.06 & 2.879 & 1 & 1 & 1 & 69 & 71 & 57 \\
36.00 & 36.00 & 2.493 & 2 & 0 & 0 & 100 & 100 & 100 \\
51.83 & 51.84 & $1.762 t$ & 2 & 2 & 0 & 56 & 51 & 95 \\
61.66 & 61.66 & $1.503 i$ & 3 & 1 & 1 & 29 & 26 & 64 \\
64.72 & 64.72 & 1.4391 & 2 & 2 & 2 & 16 & 14 & 38 \\
& & & & & & & & \\
76.35 & 76.34 & 1.2463 & 4 & 0 & 0 & 7 & 5 & 20 \\
84.68 & 84.68 & 1.1437 & 3 & 3 & 1 & 11 & 9 & 39 \\
87.42 & 87.42 & 1.1147 & 4 & 2 & 0 & 17 & 14 & 65 \\
98.39 & 98.40 & 1.0176 & 4 & 2 & 2 & 13 & 10 & 58 \\
106.81 & 106.82 & .9594 & 5 & 1 & 1 & 8 & 8 & 38 \\
& & & & & & & & \\
106.81 & & .9594 & 3 & 3 & 3 & 3 & & 13 \\
121.87 & 121.86 & .8813 & 4 & 4 & 0 & 6 & 4 & 34 \\
132.16 & 132.16 & .8427 & 5 & 3 & 1 & 17 & 9 & 115 \\
135.97 & 135.98 & .8309 & 4 & 4 & 2 & 13 & 8 & 91 \\
135.97 & & .8309 & 6 & 0 & 0 & 3 & & 23 \\
\hline
\end{tabular}


Table: A.7. Simulated X-ray powder pattern for $\mathrm{Cia}_{0.4} \mathrm{Sr}_{0.6} \mathrm{O}$

\begin{tabular}{rrllllllll}
\hline $2 \theta$ & PEAK & $d$ & $h$ & $k$ & $l$ & $l($ INT)I(PK)I(DS) \\
\hline 30.82 & 30.84 & 2.898 & 1 & 1 & 1 & 74 & 76 & 61 \\
35.74 & 35.74 & 2.510 & 2 & 0 & 0 & 100 & 100 & 100 \\
51.44 & 51.44 & 1.7749 & 2 & 2 & 0 & 57 & 53 & 97 \\
61.18 & 61.18 & 1.5137 & 3 & 1 & 1 & 31 & 28 & 68 \\
64.22 & 64.22 & 1.4492 & 2 & 2 & 2 & 17 & 15 & 39 \\
& & & & & & & & \\
75.72 & 75.72 & 1.2551 & 4 & 0 & 0 & 7 & 6 & 21 \\
83.95 & 83.96 & 1.1517 & 3 & 3 & 1 & 12 & 10 & 42 \\
86.66 & 86.66 & 1.1226 & 4 & 2 & 0 & 18 & 15 & 67 \\
97.47 & 97 & 18 & 1.0248 & 4 & 2 & 2 & 13 & 11 & 60 \\
105.74 & 105.74 & .9661 & 5 & 1 & 1 & 8 & 8 & 40 \\
& & & & & & & & \\
105.74 & & .9661 & 1 & 3 & 3 & 3 & & 13 \\
120.45 & 120.44 & .8875 & 4 & 4 & 0 & 6 & 4 & 34 \\
130.39 & 130.40 & .8486 & 5 & 3 & 1 & 17 & 10 & 116 \\
134.04 & 134.04 & .8367 & 4 & 4 & 2 & 13 & 8 & 89 \\
134.04 & & .8367 & 6 & 0 & 0 & 3 & & 22 \\
\hline
\end{tabular}

Table A.8. Simulated X-ray powder pattern for $\mathrm{Ca} 0.3 \mathrm{Sr}_{0.7} \mathrm{O}$

\begin{tabular}{lllllllrrr}
\hline $2 \theta$ & PEAK & $d$ & $h$ & $k$ & \multicolumn{3}{l}{$l(I N T) I(P K) \mid(D S)$} \\
\hline 30.61 & 30.62 & 2.919 & 1 & 1 & 1 & 78 & 81 & 64 \\
35.49 & 35.50 & 2.528 & 2 & 0 & 0 & 100 & 100 & 100 \\
51.06 & 51.06 & 1.7873 & 2 & 2 & 0 & 58 & 54 & 99 \\
60.71 & 60.72 & 1.5242 & 3 & 1 & 1 & 33 & 29 & 73 \\
63.72 & 63.72 & 1.4593 & 2 & 2 & 2 & 17 & 15 & 40 \\
& & & & & & & & \\
75.11 & 75.10 & 1.2638 & 4 & 0 & 0 & 7 & 6 & 21 \\
83.24 & 83.24 & 1.1597 & 3 & 3 & 1 & 12 & 10 & 45 \\
85.92 & 85.92 & 1.1304 & 4 & 2 & 0 & 18 & 15 & 69 \\
96.58 & 96.58 & 1.0319 & 4 & 2 & 2 & 14 & 11 & 61 \\
104.70 & 104.70 & .9729 & 5 & 1 & 1 & 8 & 8 & 42 \\
& & & & & & & & \\
104.70 & & .9729 & 3 & 3 & 3 & 3 & & 14 \\
119.08 & 119.08 & .8936 & 4 & 4 & 0 & 6 & 4 & 35 \\
128.71 & 128.70 & .8545 & 5 & 3 & 1 & 18 & 10 & 117 \\
132.20 & 132.20 & .8425 & 6 & 0 & 0 & 3 & 9 & 22 \\
132.20 & & .8425 & 4 & 4 & 2 & 13 & & 88 \\
\hline
\end{tabular}


Table A.9. Simulated $X$-ray powder pattern for

\begin{tabular}{lllllllrrr}
\multicolumn{8}{c}{$\mathrm{Ca}_{0.2} \mathrm{Sr}_{0.8} \mathrm{O}$} \\
\hline 20 & PEAK & $d$ & $h$ & $h$ & $l((\mathrm{NT})|(\mathrm{PK})|(\mathrm{DS})$ \\
\hline 30.39 & 30.40 & 2.939 & 1 & 1 & 1 & 82 & 85 & 67 \\
35.24 & 35.24 & 2.545 & 2 & 0 & 0 & 100 & 100 & 100 \\
50.69 & 50.68 & 1.7996 & 2 & 2 & 0 & 59 & 53 & 100 \\
60.25 & 60.26 & 1.5347 & 3 & 1 & 1 & 35 & 30 & 77 \\
63.23 & 63.24 & 1.4694 & 2 & 2 & 2 & 17 & 15 & 41 \\
& & & & & & & & \\
74.51 & 74.50 & 1.2725 & 4 & 0 & 0 & 7 & 6 & 22 \\
82.55 & 82.54 & 1.1678 & 3 & 3 & 1 & 13 & 11 & 47 \\
85.18 & 85.18 & 1.1382 & 4 & 2 & 0 & 19 & 15 & 71 \\
95.70 & 95.70 & 1.0390 & 4 & 2 & 2 & 14 & 11 & 63 \\
103.69 & 103.70 & .9796 & 5 & 1 & 1 & 9 & 9 & 43 \\
103.69 & & & 9796 & 3 & 3 & 3 & 3 & & 14 \\
117.76 & 117.76 & .8998 & 4 & 4 & 0 & 6 & 4 & 35 \\
127.09 & 127.10 & .8604 & 5 & 3 & 1 & 18 & 10 & 118 \\
130.46 & 130.46 & .8484 & 6 & 0 & 0 & 3 & 9 & 22 \\
130.46 & .8484 & 4 & 4 & 2 & 12 & & 86 \\
\hline
\end{tabular}

Table A.10. Simulated $X$-ray powder pattern for $\mathrm{Ca}_{0.1} \mathrm{Sr}_{0.9} \mathrm{O}$

\begin{tabular}{|c|c|c|c|c|c|c|c|c|}
\hline $2 \theta \quad 1$ & PEAK & $d$ & h & k & \multicolumn{4}{|c|}{ e $|(\mid N T)|(P K) \mid(D S)$} \\
\hline 30.18 & 30.18 & 2.959 & 1 & 1 & 18 & 86 & 89 & 70 \\
\hline 34.99 & 35.00 & 2.563 & 2 & 0 & 010 & 001 & 100 & 100 \\
\hline 50.32 & 50.32 & 1.8120 & 2 & 2 & 0 & 59 & 55 & 101 \\
\hline 59.80 & 59.80 & 1.5453 & 3 & 1 & 1 & 36 & 32 & 81 \\
\hline 62.75 & 62.76 & 1.4795 & 2 & 2 & 2 & 18 & 15 & 42. \\
\hline 73.91 & 73.92 & 1.2813 & 4 & 0 & 0 & 7 & 6 & 23 \\
\hline 81.86 & 81.86 & 1.1758 & 3 & 3 & 1 & 14 & 11 & 50 \\
\hline 84.47 & 84.46 & 1.1460 & 4 & 2 & 0 & 19 & 15 & 72 \\
\hline 94.84 & 94.84 & 1.0461 & 4 & 2 & 2 & 14 & 11 & 64 \\
\hline 102.70 & 102.70 & 9863 & 5 & 1 & 1 & 9 & 9 & 45 \\
\hline 102.70 & & .9863 & 3 & 3 & 3 & 3 & & 15 \\
\hline 116.47 & 116.48 & .9060 & 4 & 4 & 0 & 6 & 4 & 35 \\
\hline 125.54 & 125.54 & .8663 & 5 & 3 & 1 & 18 & 11 & 119 \\
\hline 128.79 & 128.80 & .8542 & 6 & 0 & 0 & 3 & 9 & 21 \\
\hline 128.79 & & .8542 & 4 & 4 & 2 & 12 & & 85 \\
\hline
\end{tabular}


Table A.11. Simulated X-ray powder pattern for Sro

\begin{tabular}{rrlllllllr}
\hline $2 \theta$ & PEAK & $d$ & $h$ & $k$ & $l($ INT)I(PK)|(DS) \\
\hline 29.97 & 29.98 & 2.979 & 1 & 1 & 1 & 89 & 93 & 73 \\
34.74 & 34.74 & 2.580 & 2 & 0 & 0 & 100 & 100 & 100 \\
49.35 & 49.96 & 1.8243 & 2 & 2 & 0 & 60 & 55 & 102 \\
59.35 & 59.36 & 1.5558 & 3 & 1 & 1 & 38 & 34 & 84 \\
62.28 & 62.28 & 1.4896 & 2 & 2 & 2 & 18 & 16 & 43 \\
& & & & & & & & \\
73.33 & 73.34 & 1.2900 & 4 & 0 & 0 & 7 & 6 & 23 \\
81.19 & 81.20 & 1.1838 & 3 & 3 & 1 & 14 & 12 & 52 \\
83.77 & 83.76 & 1.1538 & 4 & 2 & 0 & 19 & 16 & 74 \\
94.00 & 94.00 & 1.0533 & 4 & 2 & 2 & 14 & 12 & 65 \\
101.74 & 101.74 & .9930 & 5 & 1 & 1 & 9 & 9 & 46 \\
& & & & & & & \\
101.74 & & .9930 & 3 & 3 & 3 & 3 & & 15 \\
115.23 & 115.24 & .9122 & 4 & 4 & 0 & 6 & 4 & 35 \\
124.05 & 124.06 & .8722 & 5 & 3 & 1 & 18 & 11 & 119 \\
127.20 & 127.20 & .8600 & 6 & 0 & 0 & 3 & 9 & 21 \\
127.20 & & .8600 & 4 & 4 & 2 & 12 & & 84 \\
\hline
\end{tabular}


APPENDIX B: $(\mathrm{Ca}, \mathrm{Sr})_{2} \mathrm{CuO}_{3}$ 
Table B.1. Simulated X-ray powder pattern for $\mathrm{Ca}_{2} \mathrm{CuO}_{3}$

\begin{tabular}{|c|c|c|c|c|c|c|c|c|c|c|c|c|c|c|c|c|c|}
\hline $2 \theta$ & PEAK & $\bar{d}$ & $\bar{h}$ & $\bar{k}$ & & INT) & (PK) & (DS) & $2 \theta$ & $\overline{P E} \wedge \mathrm{K}$ & $d$ & $\bar{h}$ & $\mathrm{k}$ & & $\overline{\text { VT) }}$ & रK)ा & (DS) \\
\hline 14.47 & 14.48 & 6.117 & 2 & 0 & 0 & 25 & 31 & 10 & 69.07 & 69.08 & 1.3587 & 5 & 2 & 1 & 5 & 4 & 12 \\
\hline 24.65 & 24.66 & 3.609 & 1 & 1 & 0 & 2 & 2 & 1 & 72.69 & 72.70 & 1.2997 & 8 & 1 & 1 & 4 & 3 & 9 \\
\hline 28.33 & 28.34 & 3.148 & 1 & 0 & 1 & 18 & 19 & 13 & 74.06 & 74.06 & 1,2790 & 9 & 1 & 0 & 2 & 2 & 6 \\
\hline 29.17 & 29.18 & 3.059 & 4 & 0 & 0 & 1 & 1 & 1 & 74.26 & 74.28 & 1.2760 & 5 & 1 & 2 & 2 & 3 & 5 \\
\hline 32.28 & 32.28 & 2.771 & 3 & 1 & 0 & 45 & 46 & 39 & 74.50 & 74.50 & 1.2725 & 6 & 0 & 2 & 8 & 7 & 20 \\
\hline 35.24 & 35.24 & 2.545 & 3 & 0 & 1 & 67 & 67 & 64 & 75.76 & 75.76 & 1.2545 & 9 & 0 & 1 & 3 & 3 & 8 \\
\hline 36.39 & 36.40 & 2.467 & 0 & 1 & 1 & 100 & 100 & 100 & 77.30 & 77.30 & 1.2333 & 0 & 2 & 2 & 8 & 7 & 23 \\
\hline 39.35 & 39.36 & 2.288 & 2 & 1 & 1 & 0 & 0 & 0 & 78.05 & 78.04 & 1.2234 & 10 & 0 & 0 & 0 & 0 & 0 \\
\hline 44.06 & 44.06 & 2.054 & 5 & 1 & 0 & 7 & 7 & 8 & 79.16 & 79.16 & 1.2090 & 2 & 2 & 2 & 1 & 1 & 2 \\
\hline 44.39 & 44.40 & 2.039 & 6 & 0 & 0 & 30 & 29 & 38 & 79.64 & 79.64 & 1.2029 & 3 & 3 & 0 & 3 & 3 & 9 \\
\hline 46.38 & 46.38 & 1.9563 & 5 & 0 & 1 & 12 & 12 & 17 & 80.81 & 80.80 & 1.1884 & 8 & 2 & 0 & 2 & 2 & 6 \\
\hline 47.30 & 47.30 & 1.9200 & 4 & 1 & 1 & 5 & 5 & 6 & 81.99 & 81.98 & 1.1743 & 0 & 3 & 1 & 5 & 4 & 16 \\
\hline 48.15 & 48.14 & 1.8885 & 0 & 2 & 0 & 27 & 24 & 38 & 83.82 & 83.82 & 1.1532 & 2. & 3 & 1 & 0 & 0 & 0 \\
\hline 50.54 & 50.54 & 1.8044 & 2 & 2 & 0 & 2 & 2 & 3 & 85.36 & 85.36 & 1.1363 & 7 & 1 & 2 & 0 & 0 & 0 \\
\hline 56.45 & 56.46 & 1.6287 & 0 & 0 & 2 & 14 & 13 & 26 & 86.96 & 86.96 & 1.1195 & 5 & 3 & 0 & 1 & 1 & 2 \\
\hline 56.81 & 56.80 & 1.6194 & 1 & 2 & 1 & 2 & 3 & 4 & 87.41 & 87.42 & 1.1148 & 8 & 0 & 2 & 2 & 1 & 5 \\
\hline 58.11 & 58.70 & 1.5861 & 7 & 1 & 0 & 0 & 27 & 0 & 89.28 & 89.30 & 1.0963 & 4 & 3 & 1 & 0 & 1 & 1 \\
\hline 58.61 & & 1,5738 & 2 & 0 & 2 & 1 & & 2 & 89.31 & & 1.0960 & 10 & 1 & 1 & 1 & & 3 \\
\hline 58.70 & & 1.5716 & 6 & 1 & 1 & 29 & & 56 & & & & & & & & & \\
\hline 60.02 & 60.04 & 1.5400 & 7 & 0 & 1 & 0 & 0 & 0 & & & & & & & & & \\
\hline 60.49 & 60.50 & 1.5293 & 8 & 0 & 0 & 3 & 3 & 6 & & & & & & & & & \\
\hline 61.05 & 61.06 & 1.5166 & 3 & 2 & 1 & 22 & 19 & 44 & & & & & & & & & \\
\hline 62.52 & 62.52 & 1.4845 & 1 & 1 & 2 & 0 & 0 & 0 & & & & & & & & & \\
\hline 66.54 & 66.54 & 1.4041 & 3 & 1 & 2 & 11 & 10 & 25 & & & & & & & & & \\
\hline 67.56 & 67.56 & 1.3855 & 6 & 2 & 0 & 11 & 10 & 24 & & & & & & & & & \\
\hline
\end{tabular}


Table B.2. Simulated X-ray powder pattern for $\mathrm{Ca}_{1.9} \mathrm{Sr}_{0.1} \mathrm{CuO}_{3}$

\begin{tabular}{|c|c|c|c|c|c|c|c|c|c|c|c|c|c|c|c|c|c|}
\hline $2 \theta$ & PEAK & d & $\mathrm{h}$ & k & $\operatorname{ll|N}$ & $\sqrt{T) \mid(F}$ & $\overline{P K) I(1}$ & (DS) & $2 \theta$ & Fid & $\bar{d}$ & $\mathrm{~h}$ & k & & NT)i & Fk)1 & (DS) \\
\hline 14.44 & 14.46 & 6.128 & 2 & 0 & $0 \quad 3$ & 30 & 37 & 12 & 67.42 & 67.42 & 1,3880 & 6 & 2 & 0 & 10 & 9 & 24 \\
\hline 24.61 & 24.62 & 3.615 & 1 & 1 & 0 & 1 & 1 & 1 & 68.91 & 68.92 & 1,3615 & 5 & 2 & 1 & 5 & 4 & 12 \\
\hline 28.23 & 28.24 & 3.159 & 1 & 0 & 11 & 15 & 16 & 11 & 72.51 & 72.52 & 1,3025 & 8 & 1 & 1 & 5 & 4 & 14 \\
\hline 29.12 & 29.12 & 3.064 & 4 & 0 & 0 & 2 & 2 & 1 & 73.91 & 74.02 & 1.2814 & 9 & 1 & 0 & 2 & 3 & 5 \\
\hline 32.22 & 32.22 & 2.776 & 3 & 1 & $\begin{array}{ll}0 & 4\end{array}$ & 46 & 47 & 40 & 74.01 & & 1.2798 & 5 & 1 & 2 & 2 & & 6 \\
\hline 35.13 & 35.14 & 2.553 & 3 & 0 & 16 & 37 & 67 & 64 & 74.25 & 74.24 & 1.2763 & 6 & 0 & 2 & 7 & 7 & 19 \\
\hline 36.29 & 36.30 & 2.474 & 0 & 1 & 110 & 3016 & 0016 & 00 & 75.58 & 75,58 & 1.2571 & 9 & 0 & 1 & 3 & 2 & 7 \\
\hline 39.24 & 39.24 & 2.294 & 2 & 1 & 1 & 1 & 1 & 1 & 77.04 & 77.04 & 1.2368 & 0 & 2 & 2 & 8 & 7 & 23 \\
\hline 43.98 & 43.98 & 2.057 & 5 & 1 & 0 & $\theta$ & 7 & 8 & 77.88 & 77.88 & 1.2257 & 10 & 0 & 0 & 0 & 0 & 0 \\
\hline 44.31 & 44.30 & 2.043 & 6 & 0 & 02 & 27 & 28 & 35 & 78.89 & 78.90 & 1.2124 & 2 & 2 & 2 & 1 & 1 & 2 \\
\hline 46.25 & 46.26 & 1.9612 & 5 & 0 & 11 & 12 & 12 & 17 & 79.47 & 79.48 & 1.2050 & 3 & 3 & 0 & 3 & 3 & 9 \\
\hline 47.18 & 47.18 & 1.9247 & 4 & 1 & 1 & 7 & 7 & 10 & 80.63 & 80.64 & 1.1906 & 8 & 2 & 0 & 3 & 2 & 8 \\
\hline 48.06 & 48.06 & 1.8917 & 0 & 2 & 02 & 26 & 25 & 38 & 81.79 & 81.80 & 1.1766 & 0 & 3 & 1 & 5 & 4 & 16 \\
\hline 50.45 & 50.46 & 1.8075 & 2 & 2 & 0 & 2 & 2 & 3 & 83.62 & 83.62 & 1.1555 & 2 & 3 & 1 & 0 & 0 & 0 \\
\hline 56.23 & 56.24 & 1.6346 & 0 & 0 & 21 & 14 & 13 & 26 & 84.39 & 84.38 & 1.1469 & 4 & 2 & 2 & 0 & 0 & 1 \\
\hline 56.67 & 56.66 & 1.6229 & 1 & 2 & 1 & 2 & 2 & 4 & 85.07 & 85.08 & 1.1394 & 7 & 1 & 2 & 0 & 0 & 1 \\
\hline 57.18 & 57.18 & 1.6096 & 4 & 2 & 0 & 0 & 0 & 1 & 86.77 & 86.78 & 1.1214 & 5 & 3 & 0 & 1 & 1 & 2 \\
\hline 57.99 & 58.56 & 1.5890 & 7 & 1 & 0 & 0 & 25 & 0 & 87.12 & 87.12 & 1.1178 & 8 & 0 & 2 & 2 & 2 & 7 \\
\hline 58.38 & & 1.5794 & 2 & 0 & 2 & 1 & & 2 & 89.06 & 89.08 & 1.0984 & 4 & 3 & 1 & 0 & 1 & 2 \\
\hline 58.56 & & 1.5751 & 6 & 1 & 12 & 27 & & 51 & 89.08 & & 1.0982 & 10 & 1 & 1 & 1 & & 4 \\
\hline 60.37 & 60.38 & 1.5321 & 8 & 0 & 0 & 4 & 4 & & & & & & & & & & \\
\hline 60.91 & 60,90 & 1.5198 & 3 & 2 & 12 & 22 & 19 & 44 & & & & & & & & & \\
\hline 62.29 & 62.28 & 1.4894 & 1 & 1 & 2 & 0 & 0 & 0 & & & & & & & & & \\
\hline 64.57 & 64.56 & 1.4422 & 4 & 0 & 2 & 0 & 0 & 0 & & & & & & & & & \\
\hline 66.31 & 66.30 & 1.4085 & 3 & 1 & 21 & 11 & 10 & 26 & & & & & & & & & \\
\hline
\end{tabular}


Table B.3. Simulated X-ray powder pattern for $\mathrm{Ca}_{1,8} \mathrm{Sr}_{0.2} \mathrm{CuO}_{3}$

\begin{tabular}{|c|c|c|c|c|c|c|c|c|c|c|c|c|c|c|c|c|c|}
\hline $2 \theta$ & PEAK & d & h & $\mathrm{k}$ & $\bar{l} \mid \pi$ & NTाT & (PK) & (DS) & $2 \theta$ & DEAR & d & h & $\mathrm{k}$ & & (ST)! & & (DS) \\
\hline 14.42 & 14.42 & 6.140 & 2 & 0 & 0 & 27 & 33 & 10 & 68.75 & 68.74 & 1.3644 & 5 & 2 & 1 & 5 & 4 & 12 \\
\hline 24.56 & 24.58 & 3.621 & 1 & 1 & 0 & 1 & 1 & 0 & 72.34 & 72.34 & 1.3052 & 8 & 1 & 1 & 5 & 4 & 14 \\
\hline 28.13 & 28.14 & 3.170 & 1 & 0 & 1 & 13 & 14 & 10 & 73.75 & 73.76 & 1.2837 & 9 & 1 & 0 & 2 & 4 & 5 \\
\hline 29.07 & $29.0 \varepsilon$ & 3.070 & 4 & 0 & 0 & 2 & 3 & 2 & 73.76 & & 1.2836 & 5 & 1 & 2 & 2 & & 6 \\
\hline 32.16 & 32.16 & 2.781 & 3 & 1 & 0 & 47 & 48 & 41 & 73.99 & 73.98 & 1.2800 & 6 & 0 & 2 & 7 & 7 & 19 \\
\hline 35.02 & 35.02 & 2.560 & 3 & 0 & 1 & 67 & 68 & 65 & 75.39 & 75.40 & 1.2598 & 9 & 0 & 1 & 3 & 2 & 7 \\
\hline 36.18 & 36.18 & 2.481 & 0 & 1 & 110 & 001 & 100 & 100 & 76.79 & 76.78 & 1.2403 & 0 & 2 & 2 & 8 & 7 & 23 \\
\hline 39.13 & 39.14 & 2.300 & 2 & 1 & 1 & 0 & 0 & 0 & 77.71 & 77.70 & 1.2279 & 10 & 0 & 0 & 0 & 0 & 1 \\
\hline 43.90 & 43.90 & 2.061 & 5 & 1 & 0 & 6 & 7 & 8 & 78.63 & 78.64 & 1.2157 & 2 & 2 & 2 & 1 & 1 & 2 \\
\hline 44.22 & 44.22 & 2.047 & 6 & 0 & 0 & 27 & 26 & 35 & 79.31 & 79.30 & 1.2071 & 3 & 3 & 0 & 3 & 3 & 10 \\
\hline 46.13 & 46.14 & 1.9661 & 5 & 0 & 1 & 12 & 11 & 16 & 80.46 & 80.46 & 1.1927 & 8 & 2 & 0 & 3 & 2 & 8 \\
\hline 47.08 & 47.06 & 1.9294 & 4 & 1 & 1 & 8 & 7 & 11 & 81.60 & 81.60 & 1.1780 & 0 & 3 & 1 & 5 & 4 & 17 \\
\hline 47.97 & 47.98 & 1.8949 & 0 & 2 & 0 & 26 & 24 & 38 & 83.42 & 83.42 & 1.1578 & 2 & 3 & 1 & 0 & 0 & 0 \\
\hline 50.36 & 50.36 & 1.8106 & 2 & 2 & 0 & 2 & 2 & 3 & 84.11 & 84.10 & 1.1500 & 4 & 2 & 2 & 0 & 0 & 1 \\
\hline 56.01 & 56.00 & 1,6406 & 0 & 0 & 2 & 14 & 13 & 26 & 84.79 & 84.80 & 1.1425 & 7 & 1 & 2 & 0 & 0 & 1 \\
\hline 56.54 & 56.54 & 1.6265 & 1 & 2 & 1 & 2 & 2 & 3 & 86.58 & 86.58 & 1.1234 & 5 & 3 & 0 & 1 & 1 & 2 \\
\hline 57.07 & 57.08 & 1.6125 & 4 & 2 & 0 & 0 & 1 & 1 & 86.83 & 86.84 & 1.1208 & 8 & 0 & 2 & 2 & 2 & 7 \\
\hline 57.88 & 57.90 & 1.5919 & 7 & 1 & 0 & 0 & 1 & 1 & 88.84 & 88.84 & 1.1006 & 4 & 3 & 1 & 1 & 1 & 2 \\
\hline 58.16 & 58.42 & 1.5850 & 2 & 0 & 2 & 1 & 24 & 2 & 88.85 & & 1.1005 & & 1 & 1 & 1 & & 4 \\
\hline 58.41 & & 1.5786 & 6 & 1 & 1 & 27 & & 51 & & & & & & & & & \\
\hline 60.25 & 60.24 & 1.5349 & 8 & 0 & 0 & 4 & 4 & 8 & & & & & & & & & \\
\hline 60.76 & 60.76 & 1.5231 & 3 & 2 & 1 & 22 & 20 & 45 & & & & & & & & & \\
\hline 64.33 & 64.34 & 1.4469 & 4 & 0 & 2 & 0 & 0 & 1 & & & & & & & & & \\
\hline 66.07 & 66.08 & 1.4130 & 3 & 1 & 2 & 12 & 10 & 27 & & & & & & & & & \\
\hline 67.28 & 67.28 & 1.3904 & 6 & 2 & 0 & 10 & 9 & 24 & & & & & & & & & \\
\hline
\end{tabular}


Table B.4. Simulated $\mathrm{X}$-ray powder pattern for $\mathrm{Ca}_{1.7} \mathrm{Sr}_{0.3} \mathrm{CuO}_{3}$

\begin{tabular}{|c|c|c|c|c|c|c|c|c|c|c|c|c|c|c|c|c|c|}
\hline $2 \theta$ & PEAK & $d$ & $\bar{h}$ & $\bar{k}$ & $\sqrt{11(11}$ & INTI! & $(\mathrm{PK}) \mathrm{I}$ & I(DS) & $2 \theta$ & PEAK & $d$ & $\mathrm{~h}$ & k & $\ell \mid(|1|$ & $\sqrt{\text { VTII }}$ & DK)ाl & (DS) \\
\hline 14.39 & 14.40 & 6.151 & 2 & 0 & 0 & 24 & 29 & 9 & 68.58 & 68.58 & 1.3672 & 5 & 2 & 1 & 5 & 4 & 11 \\
\hline 24.52 & 24.52 & 3.628 & 1 & 1 & 0 & 0 & 0 & 0 & 72.16 & 72.16 & 1.3080 & 8 & 1 & 1 & 5 & 4 & 14 \\
\hline 28.03 & 28.04 & 3.181 & 1 & 0 & 1 & 11 & 12 & 8 & 73.51 & 73.74 & 1.2873 & 5 & 1 & 2 & 2 & 7 & 5 \\
\hline 29.01 & 29.02 & 3.075 & 4 & 0 & 0 & 3 & 3 & 2 & 73.59 & & 1.2860 & 9 & 1 & 0 & 2 & & 5 \\
\hline 32.10 & 32.12 & 2.786 & 3 & 1 & 0 & 48 & 48 & 42 & 73.74 & & 1.2838 & 6 & 0 & 2 & 7 & & 19 \\
\hline 34.92 & 34.92 & 2.566 & 3 & 0 & 1 & 68 & 68 & 65 & 75.21 & 75.20 & 1.2624 & 9 & 0 & 1 & 3 & 2 & 7 \\
\hline 36.08 & 36.08 & 2.488 & 0 & 1 & 11 & 1001 & 1001 & 100 & 76.53 & 76.54 & 1.2438 & 0 & 2 & 2 & 8 & 7 & 24 \\
\hline 39.03 & 39.04 & 2.306 & 2 & 1 & 1 & 0 & 0 & 0 & 77.54 & 77.54 & 1.2301 & 10 & 0 & 0 & 0 & 0 & 1 \\
\hline 43.81 & 43.82 & 2.065 & 5 & 1 & 0 & 6 & 7 & 8 & 78.37 & 78.38 & 1.2191 & 2 & 2 & 2 & 1 & 0 & 2 \\
\hline 44.14 & 44.14 & 2.050 & 6 & 0 & 0 & 26 & 25 & 34 & 79.14 & 79.14 & 1.2092 & 3 & 3 & 0 & 3 & 3 & 10 \\
\hline 46.01 & 46.02 & 1.9710 & 5 & 0 & 1 & 11 & 11 & 15 & 80.29 & 80.28 & 1.1948 & 8 & 2 & 0 & 3 & 2 & 8 \\
\hline 46.94 & 46.94 & 1.9341 & 4 & 1 & 1 & 8 & 8 & 12 & 81.40 & 81.40 & 1.1812 & 0 & 3 & 1 & 5 & 4 & 17 \\
\hline 47.88 & 47.88 & 1.8982 & 0 & 2 & 0 & 26 & 24 & 38 & 83.83 & 83.84 & 1.1531 & 4 & 2 & 2 & 0 & 0 & 1 \\
\hline 50.26 & 50.26 & 1.8138 & 2 & 2 & 0 & 2 & 1 & 3 & 84.51 & 84.52 & 1.1455 & 7 & 1 & 2 & 0 & 0 & 1 \\
\hline 55.79 & 55.78 & 1.6465 & 0 & 0 & 2 & 14 & 13 & 26 & 86.40 & 86.54 & 1.1253 & 5 & 3 & 0 & 1 & 2 & 2 \\
\hline 56.40 & 56.40 & 1.6300 & 1 & 2 & 1 & 1 & 1 & 2 & 86.54 & & 1.1238 & 8 & 0 & 2 & 2 & & 7 \\
\hline 56.96 & 56.96 & 1.6153 & 4 & 2 & 0 & 1 & 1 & 1 & 88.62 & 88.62 & 1.1027 & 10 & 1 & 1 & 1 & 2 & 5 \\
\hline 57.76 & 57.94 & 1.5948 & 7 & 1 & 0 & 0 & 2 & 1 & 88.62 & & 1.1027 & 4 & 3 & 1 & 1 & & 2 \\
\hline 57.93 & & 1.5905 & 2 & 0 & 2 & 1 & & 2 & & & & & & & & & \\
\hline 58.27 & 58.28 & 1.5821 & 6 & 1 & 1 & 27 & 24 & 52 & & & & & & & & & \\
\hline 60.13 & 60.12 & 1.5377 & 8 & 0 & 0 & 4 & 4 & 8 & & & & & & & & & \\
\hline 60.62 & 60.62 & 1.5263 & 3 & 2 & 1 & 22 & 20 & 46 & & & & & & & & & \\
\hline 64.10 & 64.10 & 1.4516 & 4 & 0 & 2 & 0 & 0 & 1 & & & & & & & & & \\
\hline 65.84 & $\begin{array}{l}+65.84 \\
\end{array}$ & 1.4174 & 3 & 1 & 2 & 12 & 10 & 28 & & & & & & & & & \\
\hline 67.15 & 67.16 & 1.3929 & 6 & 2 & 0 & 10 & 9 & 24 & & & & & & & & & \\
\hline
\end{tabular}


Table B.5. Simulated X-ray powder pattern for $\mathrm{Ca}_{1.6} \mathrm{Sr}_{0.4} \mathrm{CuO}_{3}$

\begin{tabular}{|c|c|c|c|c|c|c|c|c|c|c|c|c|c|c|c|c|c|}
\hline $2 \theta$ & PEAK & $d$ & h & $k$ & $\operatorname{ll}(1$ & INT)! & $|(P K)|$ & I(DS) & $2 \theta$ & PEAK & d & $h$ & k & $\operatorname{lll}$ & VT)I & $P^{\prime}(i)$ & (DS) \\
\hline 14.36 & 14.38 & 6.162 & 2 & 0 & 0 & 21 & 26 & 8 & 71.99 & 71.98 & 1.3107 & 8 & 1 & 1 & 5 & 4 & 14 \\
\hline 27.93 & 27.94 & 3.192 & 1 & 0 & 1 & 9 & 10 & 7 & 73.26 & 73.28 & 1.2910 & 5 & 1 & 2. & 2 & 2 & 5 \\
\hline 28.96 & 28.96 & 3.081 & 4 & 0 & 0 & 3 & 4 & 3 & 73.44 & 73.48 & 1.2884 & 9 & 1 & 0 & 2 & 8 & 5 \\
\hline 32.05 & 32.06 & 2.791 & 3 & 1 & 0 & 49 & 50 & 43 & 73.49 & & 1.2875 & 6 & 0 & 2 & 7 & & 19 \\
\hline 34.81 & 34.82 & 2.575 & 3 & 0 & 1 & 68 & 69 & 60 & 75.02 & 75.02 & 1.2650 & 9 & 0 & 1 & 3 & 2 & 7 \\
\hline 35.97 & 35.98 & 2.495 & 0 & 1 & 11 & 100 & 1001 & 100 & 76.28 & 76.28 & 1.2473 & 0 & 2 & 2 & 8 & 7 & 24 \\
\hline 38.92 & 38.92 & 2.312 & 2 & 1 & 1 & 0 & 0 & 0 & 77.37 & 77.38 & 1.2324 & 10 & 0 & 0 & 0 & 0 & 1 \\
\hline 43.73 & 43.74 & 2.068 & 5 & 1 & 0 & 6 & 6 & 7 & 78.12 & 78.12 & 1.2225 & 2 & 2 & 2 & 0 & 0 & 1 \\
\hline 44.05 & 44.06 & 2.054 & 6 & 0 & 0 & 26 & 25 & 34 & 78.98 & 78.98 & 1.2112 & 3 & 3 & 0 & 3 & 3 & 10 \\
\hline 45.89 & 45.90 & 1.9758 & 5 & 0 & 1 & 11 & 10 & 15 & 79.62 & 79.62 & 1.2032 & 7 & 2 & 1 & 0 & 0 & 0 \\
\hline 46.82 & 46.82 & 1.9387 & 4 & 1 & 1 & 9 & 9 & 13 & 80.11 & 80.12 & 1.1970 & 8 & 2 & 0 & 3 & 2 & 8 \\
\hline 47.80 & 47.80 & 1.9014 & 0 & 2 & 0 & 26 & 24 & 38 & 81.21 & 81.22 & 1.1835 & 0 & 3 & 1 & 5 & 4 & 17 \\
\hline 50.17 & 50.18 & 1.8169 & 2 & 2 & 0 & 1 & 1 & 2 & 83.56 & 83.56 & 1.1561 & 4 & 2 & 2 & 0 & 0 & 1 \\
\hline 55.57 & 55.58 & 1.6525 & 0 & 0 & 2 & 15 & 13 & 26 & 84.23 & 84.24 & 1.1486 & 7 & 1 & 2 & 0 & 0 & 1 \\
\hline 56.27 & 56.26 & 1.6336 & 1 & 2 & 1 & 1 & 1 & 2 & 86.21 & 86.24 & 1.1273 & 5 & 3 & 0 & 1 & 2 & 2 \\
\hline 56.86 & 56.86 & 1.6181 & 4 & 2 & 0 & 1 & 1 & 1 & 86.25 & & 1.1268 & 8 & 0 & 2 & 2 & & 7 \\
\hline 57.65 & 57.72 & 1.5977 & 7 & 1 & 0 & 1 & 2 & 1 & 88.40 & 88.40 & 1.1049 & 10 & 1 & 1 & 1 & 2 & 5 \\
\hline 57.71 & & 1.5961 & 2 & 0 & 2 & 1 & & 2 & 88.41 & & 1.1048 & 4 & 3 & 1 & 1 & & 3 \\
\hline 58.13 & 58.14 & 1.5856 & 6 & 1 & 1 & 27 & 23 & 52 & & & & & & & & & \\
\hline 60.00 & 60.00 & 1.5405 & 8 & 0 & 0 & 4 & 4 & 8 & & & & & & & & & \\
\hline 60.48 & 60.48 & 1.5296 & 3 & 2 & 1 & 23 & 20 & 47 & & & & & & & & & \\
\hline 63.87 & 63.88 & 1.4563 & 4 & 0 & 2 & 0 & 0 & 1 & & & & & & & & & \\
\hline 65.60 & 65.60 & 1.4219 & 3 & 1 & 2 & 12 & 11 & 28 & & & & & & & & & \\
\hline 67.02 & 67.02 & 1.3953 & 6 & 2 & 0 & 10 & 9 & 24 & & & & & & & & & \\
\hline 68.42 & 68.42 & 1.3701 & 5 & 2 & 1 & 4 & 4 & 11 & & & & & & & & & \\
\hline
\end{tabular}


Table B.6. Simulated $\mathrm{X}$-ray powder pattern for $\mathrm{Ca}_{1.5} \mathrm{Sr}_{(.5} \mathrm{CuO}_{3}$

\begin{tabular}{|c|c|c|c|c|c|c|c|c|c|c|c|c|c|c|c|c|c|}
\hline $2 \theta$ & PEAK & d & $\mathrm{h}$ & k & $\ell \mid(\mathbb{N}$ & VT)।(F & $P K) \mid([$ & (DS) & $2 \theta \quad F$ & PEAK & d & $\mathrm{h}$ & k & $\ell|| \mid$ & NTIII & DK)I & \\
\hline 14.34 & $\begin{array}{l}4 \\
14.34\end{array}$ & 6.173 & 2 & 0 & $0 \quad 3$ & $39 \quad 4$ & $47 \quad 1$ & 15 & 71.81 & 71.82 & 1.3135 & 8 & 1 & 1 & 7 & 6 & 20 \\
\hline 24.43 & $3 \quad 24.44$ & 3.640 & 1 & 1 & 0 & 4 & 4 & 2 & 73.01 & 73.02 & 1.2948 & 5 & 1 & 2 & 4 & 4 & 10 \\
\hline 27.83 & 27.84 & 3.203 & 1 & 0 & $\begin{array}{ll}1 & 1\end{array}$ & $17 \quad 1$ & $18 \quad 1$ & 12 & 73.25 & 73.24 & 1.2913 & 6 & 0 & 2 & 8 & 10 & 22 \\
\hline 28.90 & 28.90 & 3.087 & 4 & 0 & 0 & 0 & 1 & 0 & 73.28 & & 1.2907 & 9 & 1 & 0 & 3 & & 8 \\
\hline 31.99 & 32.00 & 2.796 & 3 & 1 & $0 \quad 5$ & $88 \quad 6$ & $60 \quad 5$ & 51 & 74.84 & 74.84 & 1.2677 & 9 & 0 & 1 & 4 & 3 & 10 \\
\hline 34.71 & 34.72 & 2.583 & 3 & 0 & 17 & 737 & 747 & 70 & 75.16 & 75.06 & 1.2631 & 1 & 3 & 0 & 0 & 2 & 1 \\
\hline 35.87 & 35.88 & 2.501 & 0 & 1 & 110 & 00 & 0010 & 00 & 76.03 & 76.04 & 1.2507 & 0 & 2 & 2 & 9 & 8 & 26 \\
\hline 38.81 & 38.82 & 2.318 & 2 & 1 & 1 & 4 & 4 & 4 & 77.86 & 77.86 & 1.2258 & 2 & 2 & 2 & 2 & 1 & 5 \\
\hline 43.65 & 43.66 & 2.072 & 5 & 1 & 0 & $9 \quad 1$ & 101 & 12 & 78.82 & 78.82 & 1.2133 & 3 & 3 & 0 & 4 & 4 & 13 \\
\hline 43.97 & 43.96 & 2.058 & 6 & 0 & 02 & 262 & 253 & 34 & 79.42 & 79.42 & 1.2056 & 7 & 2 & 1 & 0 & 0 & 0 \\
\hline 45.77 & 45.78 & 1.9807 & 5 & 0 & 11 & 141 & 131 & 19 & 79.94 & 79.94 & 1.1991 & 8 & 2 & 0 & 3 & 3 & 11 \\
\hline 46.70 & 46.70 & 1.9434 & 4 & 1 & 1 & 2 & 2 & 3 & 81.02 & 81.02 & 1.1858 & 0 & 3 & 1 & 6 & 5 & 20 \\
\hline 47.71 & 47.72 & 1.9046 & 0 & 2 & 02 & $26 \quad 2$ & 243 & 38 & 82.82 & 82.82 & 1.1645 & 2 & 3 & 1 & 1 & 1 & 2 \\
\hline 50.08 & 50.08 & 1.8200 & 2 & 2 & 0 & 3 & 3 & 5 & 85.97 & 85.98 & 1.1298 & 8 & 0 & 2 & 3 & 3 & 9 \\
\hline 55.35 & 55.36 & 1.6584 & 0 & 0 & 21 & $15 \quad 1$ & 132 & 27 & 86.02 & & 1.1292 & 5 & 3 & 0 & 1 & & 4 \\
\hline 56.14 & 56.14 & 1.6371 & 1 & 2 & 1 & 3 & 3 & 6 & 88.18 & 88.18 & 1.1071 & 10 & 1 & 1 & 0 & 0 & 1 \\
\hline 57.49 & 57.50 & 1.6017 & 2 & 0 & 2 & 2 & 2 & 4 & 88.77 & 88.78 & 1.1012 & 1 & 0 & 3 & 0 & 0 & 1 \\
\hline 57.99 & 58.00 & 1.5892 & 6 & 1 & 13 & 302 & 275 & 59 & & & & & & & & & \\
\hline 59.29 & 59.30 & 1.5573 & 7 & 0 & 1 & 0 & 0 & 0 & & & & & & & & & \\
\hline 59.88 & 59.88 & 1.5433 & 8 & 0 & 0 & 5 & 51 & 10 & & & & & & & & & \\
\hline 60.33 & 60.34 & 1.5329 & 3 & 2 & 12 & 26 & 235 & 54 & & & & & & & & & \\
\hline 61.38 & 61.38 & 1.5092 & 1 & 1 & 2 & 1 & 1 & 2 & & & & & & & & & \\
\hline 65.37 & 65.38 & 1.4263 & 3 & 1 & 21 & 16 & 133 & 36 & & & & & & & & & \\
\hline 66.88 & 66.88 & 1.3978 & 6 & 2 & 01 & 11 & 10 & 27 & & & & & & & & & \\
\hline 68.26 & 68.26 & 1.3729 & 5 & 2 & 1 & 7 & 61 & 17 & & & & & & & & & \\
\hline
\end{tabular}


Table B.7. Simulated $\mathrm{X}$-ray powder pattern for $\mathrm{Ca}_{1.4} \mathrm{Sr}_{0.6} \mathrm{CuO}_{3}$

\begin{tabular}{|c|c|c|c|c|c|c|c|c|c|c|c|c|c|c|c|c|c|}
\hline $2 \theta$ & PEAK & d & $\bar{h}$ & $k$ & & (INT)! & $\overline{I(P K)}$ & $\pi(D S)$ & $2 \theta$ & PEAK & d & $\mathrm{h}$ & k & & & I(P & (DS) \\
\hline 14.31 & 14.32 & 6.185 & 2 & 0 & 0 & 36 & 43 & 13 & 71.64 & 71.64 & 1.3162 & 8 & 1 & 1 & 7 & $\theta$ & 19 \\
\hline 24.39 & 24.40 & 3.646 & 1 & 1 & 0 & 3 & 3 & 2 & 72.77 & 72.78 & 1.2985 & 5 & 1 & 2 & 4 & . & 10 \\
\hline 27.73 & 27.74 & 3.214 & 1 & 0 & 1 & 15 & 16 & 11 & 73.00 & 73.00 & 1.2950 & 6 & 0 & 2 & $\varepsilon$ & $s$ & 22 \\
\hline 28.85 & 28.86 & 3.092 & 4 & 0 & 0 & 1 & 1 & 0 & 73.13 & 73.14 & 1.2930 & 9 & 1 & 0 & 3 & 5 & 8 \\
\hline 31.93 & 31.94 & 2.800 & 3 & 1 & 0 & 59 & 61 & 51 & 74.66 & 74.66 & 1.2703 & 9 & 0 & 1 & 4 & 3 & 10 \\
\hline 34.60 & 34.60 & 2.590 & 3 & 0 & 1 & 74 & 74 & 71 & 75.01 & 74.88 & 1.2652 & 1 & 3 & 0 & $c$ & 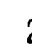 & 0 \\
\hline 35.77 & 35.78 & 2.508 & 0 & 1 & 11 & 1001 & 100 & 100 & 75.78 & 75.78 & 1.2542 & 0 & 2 & 2 & $\varsigma$ & 8 & 27 \\
\hline 38.71 & 38.70 & 2.32 .5 & 2 & 1 & 1 & 3 & 3 & 4 & 77.61 & 77.62 & 1.2292 & 2 & 2 & 2 & 1 & . & 4 \\
\hline 43.57 & 43.58 & 2.076 & 5 & 1 & 0 & 9 & 10 & 12 & 78.66 & 78.66 & 1.2154 & 3 & 3 & 0 & 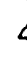 & 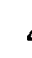 & 13 \\
\hline 43.88 & 43.88 & 2.062 & 6 & 0 & 0 & 26 & 25 & 34 & 79.77 & 79.78 & 1.2012 & 8 & 2 & 0 & 3 & 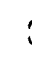 & 10 \\
\hline 45.65 & 45.66 & 1.9856 & 5 & 0 & 1 & 13 & 13 & 19 & 80.83 & 80.84 & 1.1881 & 0 & 3 & 1 & 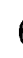 & 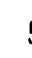 & 20 \\
\hline 46.58 & 46.58 & 1.9481 & 4 & 1 & 1 & 3 & 3 & 4 & 82.63 & 82.62 & 1.1668 & 2 & 3 & 1 & . & & 2 \\
\hline 47.63 & 47.62 & 1.9079 & 0 & 2 & 0 & 25 & 24 & 37 & 85.69 & 85.70 & 1.1328 & 8 & 0 & 2 & & 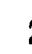 & 9 \\
\hline 49.99 & 50.00 & 1.8231 & 2 & 2 & 0 & 3 & 3 & 5 & 85.84 & 85.84 & 1.1312 & 3 & 3 & 0 & . & 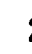 & 4 \\
\hline 55.14 & 55.14 & 1.6644 & 0 & 0 & 2 & 15 & 14 & 27 & 87.95 & 87.96 & 1.1094 & 10 & 1 & 1 & & r & 1 \\
\hline 56.00 & 56.00 & 1.6407 & 1 & 2 & 1 & 3 & 3 & 5 & 88.37 & 88.36 & 1.1052 & 1 & 0 & 3 & ( & ( & 1 \\
\hline 57.28 & 57.28 & 1.6072 & 2 & 0 & 2 & 2 & 2 & 4 & & & & & & & & & \\
\hline 57.85 & 57.86 & 1.5927 & 6 & 1 & 1 & 30 & 27 & 59 & & & & & & & & & \\
\hline 59.15 & 59.16 & 1.5607 & 7 & 0 & 1 & 0 & 0 & 0 & & & & & & & & & \\
\hline 59.76 & 59.76 & 1.5461 & 8 & 0 & 0 & 5 & 5 & 9 & & & & & & & & & \\
\hline 60.19 & 60.20 & 1.5361 & 3 & 2 & 1 & 26 & 24 & 54 & & & & & & & & & \\
\hline 61.16 & 61.16 & 1.5141 & 1 & 1 & 2 & 1 & 1 & 1 & & & & & & & & & \\
\hline 65.15 & 65.14 & 1.4308 & 3 & 1 & 2 & 16 & 14 & 37 & & & & & & & & & \\
\hline 66.75 & 66.76 & 1.4002 & 6 & 2 & 0 & 11 & 10 & 27 & & & & & & & & & \\
\hline 68.10 & 68.10 & 1.3757 & 5 & 2 & 1 & 7 & 6 & 16 & & & & & & & & & \\
\hline
\end{tabular}


Table B.8. Simulated $\mathrm{X}$-ray powder pattern for $\mathrm{Ca}_{1.3} \mathrm{Sr}_{0.7} \mathrm{CuO}_{3}$

\begin{tabular}{|c|c|c|c|c|c|c|c|c|c|c|c|c|c|c|c|c|c|}
\hline $2 \theta$ & PEAK & d & $\mathrm{h}$ & k & $\ell \mid(I N)$ & NT)।(F & $\mathrm{PK}) \mid([$ & (DS) & $2 \theta$ & PEAK & $d$ & & $k$ & l! & VT)I( & DK)I & (DS) \\
\hline 14.28 & 14.30 & 6.196 & 2 & 0 & 03 & 334 & 401 & 12 & 72.53 & 72.54 & 1.3023 & 5 & 1 & 2 & 3 & 4 & 9 \\
\hline 24.35 & 24.36 & 3.652 & 1 & 1 & 0 & 2 & 2 & 1 & 72.76 & 72.76 & 1.2987 & 6 & 0 & 2 & 8 & 9 & 22 \\
\hline 27.63 & 27.64 & 3.226 & 1 & 0 & 11 & 131 & 141 & 10 & 72.98 & 72.96 & 1.2954 & 9 & 1 & 0 & 3 & 6 & 8 \\
\hline 28.80 & 28.80 & 3.098 & 4 & 0 & 0 & 1 & 1 & 1 & 74.48 & 74.48 & 1.2730 & 9 & 0 & 1 & 4 & 3 & 10 \\
\hline 31.87 & 31.88 & 2.805 & 3 & 1 & 06 & 606 & 625 & 52 & 74.86 & 74.70 & 1.2674 & 1 & 3 & 0 & 0 & 2 & 0 \\
\hline 34.50 & 34.50 & 2.597 & 3 & 0 & 17 & 747 & 767 & 71 & 75.54 & 75.54 & 1.2577 & 0 & 2 & 2 & 9 & 8 & 27 \\
\hline 35.67 & 35.68 & 2.515 & 0 & 1 & 1 tc & 0010 & 0010 & 00 & 77.36 & 77.36 & 1.2325 & 2 & 2 & 2 & 1 & 1 & 4 \\
\hline 38.60 & 38.60 & 2.331 & 2 & 1 & 1 & 3 & 3 & 3 & 78.50 & 78.50 & 1.2175 & 3 & 3 & 0 & 4 & 4 & 14 \\
\hline 43.48 & 43.48 & 2.079 & 5 & 1 & 0 & 9 & $\begin{array}{ll}9 & 1\end{array}$ & 11 & 79.60 & 79.60 & 1.2033 & 8 & 2 & 0 & 3 & 3 & 10 \\
\hline 43.80 & 43.80 & 2.065 & 6 & 0 & 02 & $26 \quad 2$ & $25 \quad 3$ & 34 & 80.64 & 80.64 & 1.1904 & 0 & 3 & 1 & 6 & 5 & 20 \\
\hline 45.54 & 45.54 & 1.9904 & 5 & 0 & 1 & 131 & 131 & 18 & 82.43 & 82.44 & 1.1691 & 2 & 3 & 1 & 1 & 0 & 2 \\
\hline 46.47 & 45.46 & 1.9527 & 4 & 1 & 1 & 3 & 3 & 4 & 85.41 & 85.40 & 1.1358 & 8 & 0 & 2 & 3 & 2 & 9 \\
\hline 47.54 & 47.54 & 1.9111 & 0 & 2 & 02 & $25 \quad 2$ & 243 & 37 & 85.66 & 85.66 & 1.1331 & 5 & 3 & 0 & 1 & 2 & 4 \\
\hline 49.90 & 49.90 & 1.8262 & 2 & 2 & 0 & 3 & 3 & 4 & 87.73 & 87.74 & 1.11161 & 10 & 1 & 1 & 0 & 0 & 1 \\
\hline 54.92 & 54.92 & 1.6704 & 0 & 0 & 2 & 151 & 142 & 27 & 87.77 & & 1.1112 & 4 & 3 & 1 & 0 & & 0 \\
\hline 55.87 & 55.88 & 3442 & 1 & 2 & 1 & 2 & 2 & 4 & 87.98 & 88.00 & 1.1091 & 1 & 0 & 3 & 0 & 0 & 1 \\
\hline 57.06 & 57.06 & 1.6128 & 2 & 0 & 2 & 2 & 2 & 3 & & & & & & & & & \\
\hline 57.71 & 57.72 & 1.5962 & 6 & 1 & 13 & 302 & 275 & 59 & & & & & & & & & \\
\hline 59.64 & 59.64 & 1.5489 & 8 & 0 & 0 & 5 & 5 & 9 & & & & & & & & & \\
\hline 60.05 & 60.06 & 1.5394 & 32 & 2 & 12 & $27 \quad 2$ & $24 \quad 5$ & 55 & & & & & & & & & \\
\hline 60.94 & .94 & & 11 & 1 & 2 & 1 & 1 & & & & & & & & & & \\
\hline 64.92 & 64.92 & 1.4352 & 31 & 1 & 2 & 161 & 143 & 37 & & & & & & & & & \\
\hline 66.62 & 66.62 & 1.4027 & 62 & 2 & 0 & 111 & 102 & 27 & & & & & & & & & \\
\hline 67.94 & 67.94 & 1.3785 & 52 & 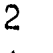 & 1 & 6 & $\begin{array}{ll}6 & 1\end{array}$ & 16 & & & & & & & & & \\
\hline 71.47 & 71.48 & 1.3189 & 8 & 1 & 1 & 7 & $\begin{array}{ll}6 & 1\end{array}$ & 19 & & & & & & & & & \\
\hline
\end{tabular}


Table B.9. Simulated $\mathrm{X}$-ray powder pattern for $\mathrm{Ca}_{1.2} \mathrm{Sr}_{0.8} \mathrm{CuO}_{3}$

\begin{tabular}{|c|c|c|c|c|c|c|c|c|c|c|c|c|c|c|c|c|c|}
\hline $2 \theta$ & PEAK & $\bar{d}$ & $h$ & $\bar{k}$ & $\operatorname{li(IN}$ & NTाK & $\overline{(\mathrm{PK}) \|(1}$ & (DS) & $2 \theta$ & $\overline{\text { PEAK }}$ & d & $\bar{h}$ & $\bar{k}$ & lा(II) & गागा। & $\overline{D K M)}$ & DS) \\
\hline 14.26 & 14.26 & 6.207 & 2 & 0 & 03 & 30 & 36 & 11 & 67.78 & 67.78 & 1.3814 & 5 & 2 & 1 & 6 & 5 & 15 \\
\hline 24.31 & 24.32 & 3.659 & 1 & 1 & 0 & 1 & 2 & 1 & 71.30 & 71.30 & 1.3217 & 8 & 1 & 1 & 7 & 6 & 19 \\
\hline 27.54 & 27.54 & 3.237 & 1 & 0 & 11 & 12 & 12 & 8 & 72.29 & 72.30 & 1.3060 & 5 & 1 & 2 & 3 & 4 & 9 \\
\hline 28.74 & 28.74 & 3.103 & 4 & 0 & 0 & 1 & 1 & 1 & 72.51 & 72.52 & 1.3025 & 6 & 0 & 2 & 8 & 8 & 22 \\
\hline 31.82 & 31.82 & 2.810 & 3 & 1 & 06 & 60 & 63 & 52 & 72.82 & 72.72 & 1.2977 & 9 & 1 & 0 & 3 & 5 & 8 \\
\hline 34.40 & 34.40 & 2.605 & 3 & 0 & 17 & 74 & 76 & 71 & 74.30 & 74.30 & 1.2756 & 9 & 0 & 1 & 4 & 3 & 10 \\
\hline 35.56 & 35.56 & 2.522 & 0 & 1 & 110 & 001 & 10010 & 00 & 74.71 & 74.50 & 1.2695 & 1 & 3 & 0 & 0 & 2 & 0 \\
\hline 38.49 & 38.50 & 2.337 & 2 & 1 & 1 & 3 & 3 & 3 & 75.29 & 75.30 & 1.2612 & 0 & 2 & 2 & 9 & 8 & 27 \\
\hline 43.40 & 43.40 & 2.083 & 5 & 1 & 0 & 8 & 9 & 11 & 77.11 & 77.12 & 1.2359 & 2 & 2 & 2 & 1 & 1 & 4 \\
\hline 43.72 & 43.72 & 2.069 & 6 & 0 & 02 & 25 & 25 & 34 & 78.34 & 78.34 & 1.2195 & 3 & 3 & 0 & 4 & 4 & 14 \\
\hline 45.42 & 45.42 & 1.9953 & 5 & 0 & 11 & 13 & 12 & 18 & 79.44 & 79.44 & 1.2055 & 8 & 2 & 0 & 3 & 3 & 10 \\
\hline 46.35 & 46.36 & 1.9574 & 4 & 1 & 1 & 4 & 4 & 5 & 80.45 & 80.46 & 1.1927 & 0 & 3 & 1 & 6 & 5 & 21 \\
\hline 47.45 & 47.46 & 1.9144 & 0 & 2 & 02 & 25 & 24 & 38 & 82.24 & 82.24 & 1.1713 & 2 & 3 & 1 & 1 & 0 & 2 \\
\hline 49.81 & 49.80 & 1.8293 & 2 & 2 & 0 & 3 & 2 & 4 & 85.13 & 85.14 & 1.1388 & 8 & 0 & 2 & 3 & 2 & 9 \\
\hline 54.71 & 54.72 & 1.6763 & 0 & 0 & 21 & 15 & 14 & 28 & 85.48 & 85.40 & 1.1351 & 5 & 3 & 0 & 1 & 2 & 4 \\
\hline 55.74 & 55.74 & 1.6477 & 1 & 2 & 1 & 2 & 2 & 4 & 87.51 & 87.52 & 1.1138 & 10 & 1 & 1 & 0 & 1 & 2 \\
\hline 56.43 & 56.44 & 1.6293 & 4 & 2 & 0 & 0 & 0 & 0 & 87.56 & & 1.1133 & 4 & 3 & 1 & 0 & & 1 \\
\hline 56.85 & 56.84 & 1.6183 & 2 & 0 & 2 & 2 & 2 & 3 & 87.59 & & 1.1131 & 1 & 0 & 3 & 0 & & 1 \\
\hline 57.20 & 57.58 & 1.6092 & 7 & 1 & 0 & 0 & 27 & 0 & & & & & & & & & \\
\hline 57.57 & & 1.5997 & 6 & 1 & 13 & 30 & & 59 & & & & & & & & & \\
\hline 59.53 & 59.52 & 1.5517 & 8 & 0 & 0 & 4 & 5 & 9 & & & & & & & & & \\
\hline 59.91 & 59.92 & 1.5426 & 3 & 2 & 12 & 27 & 24 & 56 & & & & & & & & & \\
\hline 60.72 & 60.72 & 1.5240 & 1 & 1 & 2 & 0 & 1 & 1 & & & & & & & & & \\
\hline 64.70 & 64.70 & 1.4397 & 3 & 1 & 21 & 16 & 14 & 38 & & & & & & & & & \\
\hline 66.49 & 66.48 & 1.4051 & 6 & 2 & $\begin{array}{ll}0 & 1\end{array}$ & 11 & 10 & 27 & & & & & & & & & \\
\hline
\end{tabular}


Table B.10. Simulated X-ray powder pattern for $\mathrm{Ca}_{1.1} \mathrm{Sr}_{0.9} \mathrm{CuO}_{3}$

\begin{tabular}{|c|c|c|c|c|c|c|c|c|c|c|c|c|c|c|c|c|c|}
\hline $2 \theta$ & PEAK & $d$ & $\bar{h}$ & k & $\operatorname{l|l|1}$ & $\sqrt{\operatorname{VI}}$ & $(P K) \|$ & (DS) & $2 \theta$ & PEAK & d & $\mathrm{h}$ & k & & $\sqrt{\mathrm{VT} I}$ & कK)ा & DS) \\
\hline 14.23 & 14.24 & 6.218 & 2 & 0 & 02 & 28 & 34 & 10 & 67.63 & 67.62 & 1.3842 & 5 & 2 & 1 & 6 & 5 & 15 \\
\hline 24.27 & 24.28 & 3.665 & 1 & 1 & 0 & 1 & 1 & 1 & 71.13 & 71.14 & 1.3244 & 8 & 1 & 1 & 7 & 6 & 19 \\
\hline 27.44 & 27.44 & 3.248 & 1 & 0 & 11 & 10 & 11 & 7 & 72.05 & 72.06 & 1.3097 & 5 & 1 & 2 & 3 & 3 & 9 \\
\hline 28.69 & 28.70 & 3.109 & 4 & 0 & 0 & 1 & 2. & 1 & 72.27 & 72.28 & 1.3062 & 6 & 0 & 2 & 8 & 8 & 22 \\
\hline 31.76 & 31.76 & 2.815 & 3 & 1 & 0 & 61 & 63 & 53 & 72.67 & 72.66 & 1.3000 & 9 & 1 & 0 & 3 & 3 & 8 \\
\hline 34.30 & 34.30 & 2.612 & 3 & 0 & 17 & 75 & 76 & 72. & 74.12 & 74.12 & 1.2782 & 9 & 0 & 1 & 4 & 3 & 10 \\
\hline 35.46 & 35.46 & 2.529 & 0 & 1 & 110 & 001 & $100 !$ & 100 & 75.05 & 75.06 & 1.2646 & 0 & 2 & 2 & 9 & 8 & 27 \\
\hline 38.39 & 38.40 & 2.343 & 2 & 1 & 1 & 2 & 2 & 2 & 76.54 & 76.54 & 1.2436 & 10 & 0 & 0 & 0 & 0 & 0 \\
\hline 43.32 & 43.32 & 2.087 & 5 & 1 & 0 & 8 & 9 & 11 & 76.86 & 76.86 & 1.2392 & 2 & 2 & 2 & 1 & 1 & 3 \\
\hline 43.63 & 43.64 & 2.073 & 6 & 0 & 0 & 25 & 25 & 33 & 78.18 & 78.18 & 1.2216 & 3 & 3 & 0 & 5 & 4 & 14 \\
\hline 45.30 & 45.30 & 2.000 & 5 & 0 & 1 & 12 & 12 & 17 & 79.27 & 79.26 & 1.2076 & 8 & 2 & 0 & 3 & 3 & 10 \\
\hline 46.23 & 46.24 & 1.9620 & 4 & 1 & 1 & 4 & 4 & 6 & 80.27 & 80.26 & 1.1950 & 0 & 3 & 1 & 6 & 5 & 21 \\
\hline 47.37 & 47.38 & 1.9176 & 0 & 2 & 0 & 25 & 23 & 37 & 82.05 & 82.04 & 1.1736 & 2 & 3 & 1 & 0 & 0 & 2 \\
\hline 49.72 & 49.72 & 1.8324 & 2 & 2 & 0 & 2 & 2 & 4 & 82.88 & 82.88 & 1.1639 & 7 & 1 & 2 & 0 & 0 & 0 \\
\hline 54.50 & 54.50 & 1.6823 & 0 & 0 & 2 & 15 & 14 & 28 & 84.86 & 84.86 & 1.1417 & 8 & 0 & 2 & 3 & 2 & 9 \\
\hline 55.61 & 55.62 & 1.6513 & 1 & 2 & 1 & 2 & 2 & 3 & 85.29 & 85.30 & 1.1370 & 5 & 3 & 0 & 1 & 1 & 4 \\
\hline 56.32 & 56.34 & 1.6321 & 4 & 2 & 0 & 0 & 0 & 0 & 87.20 & 87.30 & 1.1170 & 1 & 0 & 3 & 0 & 1 & 1 \\
\hline 56.63 & 56.64 & 1.6239 & 2 & 0 & 2 & 2 & 2 & 3 & 87.29 & & 1.1160 & 10 & 1 & 1 & 1 & & 2 \\
\hline 57.09 & 57.44 & 1.6121 & 7 & 1 & 0 & 0 & 27 & 0 & 87.35 & & 1.1155 & 4 & 3 & 1 & 0 & & 1 \\
\hline 57.43 & & 1.6032 & 6 & 1 & 1 & 30 & & 59 & & & & & & & & & \\
\hline 59.41 & 59.42 & 1.5546 & 8 & 0 & 0 & 4 & 5 & 9 & & & & & & & & & \\
\hline 59.78 & 59.78 & 1.5458 & 3 & 2 & 1 & 27 & 24 & 56 & & & & & & & & & \\
\hline 60.51 & 60.50 & 1.5289 & 1 & 1 & 2 & 0 & 1 & 1 & & & & & & & & & \\
\hline 64.47 & 64.48 & 1.7441 & 3 & 1 & 2 & 17 & 14 & 39 & & & & & & & & & \\
\hline 66.36 & 66.36 & 1.4076 & 6 & 2 & 0 & 11 & 10 & 27 & & & & & & & & & \\
\hline
\end{tabular}


Table B.11. Simulated X-ray powder pattern for $\mathrm{CaSrCuO}_{3}$

\begin{tabular}{|c|c|c|c|c|c|c|c|c|c|c|c|c|c|c|c|c|c|}
\hline $2 \theta$ & PEAK & $\bar{d}$ & $\bar{h}$ & $k$ & $\overline{l 11}$ & $\mid \overline{N T T \mid}$ & $\overline{|(\mathrm{PK})|}$ & I(DS) & $2 \theta$ & PEAK & $d$ & $h$ & $k$ & 911 & NT) & $\overline{\mathrm{PK})}$ & (DS) \\
\hline 14.21 & 14.22 & 6.229 & 2 & 0 & 0 & 2.5 & 31 & 9 & 66.23 & 66.22 & 1.4101 & 6 & 2 & 0 & 11 & 9 & 27 \\
\hline 24.22 & 24.24 & 3.671 & 1 & 1 & 0 & 1 & 1 & 0 & 67.47 & 67.48 & 1.3870 & 5 & 2 & 1 & 6 & 5 & 14 \\
\hline 27.34 & 27.36 & 3.259 & 1 & 0 & 1 & 9 & 9 & 6 & 70.96 & 70.96 & 1.3271 & 8 & 1 & 1 & 7 & 6 & 19 \\
\hline 28.64 & 28.64 & 3.115 & 4 & 0 & 0 & 2 & 2 & 1 & 71.81 & 71.82 & 1.3134 & 5 & 1 & 2 & 3 & 3 & 9 \\
\hline 31.70 & 31.70 & 2.820 & 3 & 1 & 0 & 62 & 63 & 54 & 72.04 & 72.04 & 1.3099 & 6 & 0 & 2 & 8 & 8 & 22 \\
\hline 34.20 & 34.20 & 2.620 & 3 & 0 & 1 & 75 & 77 & 72 & 72.52 & 72.52 & 1.3024 & 9 & 1 & 0 & 3 & 3 & 8 \\
\hline 35.36 & 35.36 & 2.536 & 0 & 1 & 1 & 100 & 100 & 100 & 73.94 & 73.94 & 1.2809 & 9 & 0 & 1 & 3 & 3 & 10 \\
\hline 38.29 & 38.30 & 2.349 & 2 & 1 & 1 & 2 & 2 & 2 & 74.81 & 74.82 & 1.2681 & 0 & 2 & 2 & 9 & 8 & 28 \\
\hline 43.24 & 43.24 & 2.091 & 5 & 1 & 0 & 8 & 8 & 10 & 76.38 & 76.40 & 1.2459 & 10 & 0 & 0 & 0 & 0 & 0 \\
\hline 43.55 & 43.56 & 2.076 & 6 & 0 & 0 & 25 & 24 & 33 & 76.62 & 76.62 & 1.2426 & 2 & 2 & 2 & 1 & 1 & 3 \\
\hline 45.19 & 45.20 & 2.005 & 5 & 0 & 1 & 12 & 11 & 17 & 78.02 & 78.02 & 1.2237 & 3 & 3 & 0 & 5 & 4 & 14 \\
\hline 46.12 & 46.12 & 1.9667 & 4 & 1 & 1 & 4 & 4 & 6 & 79.10 & 79.10 & 1.2097 & 8 & 2 & 0 & 3 & 3 & 10 \\
\hline 47.28 & 47.28 & 1.9208 & 0 & 2 & 0 & 25 & 23 & 37 & 80.08 & 80.08 & 1.1973 & 0 & 3 & 1 & 6 & 5 & 21 \\
\hline 49.63 & 49.62 & 1.8355 & 2 & 2 & 0 & 2 & 2 & 4 & 81.86 & 81.86 & 1.1758 & 2 & 3 & 1 & 0 & 0 & 1 \\
\hline 54.29 & 54.30 & 1.6882 & 0 & 0 & 2 & 15 & 14 & 28 & 82.61 & 82.62 & 1.1670 & 7 & 1 & 2 & 0 & 0 & 1 \\
\hline 55.49 & 55.48 & 1.6548 & 1 & 2 & 1 & 1 & 1 & 3 & 84.59 & 84.58 & 1.1447 & 8 & 0 & 2 & 3 & 2 & 9 \\
\hline 56.22 & 56.42 & 1.6349 & 4 & 2 & 0 & 0 & 2 & 0 & 85.11 & 85.12 & 1.1390 & 5 & 3 & 0 & 1 & 1 & 3 \\
\hline 56.42 & & 1.6295 & 2 & 0 & 2 & 1 & & 3 & 86.82 & 86.82 & 1.1209 & 1 & 0 & 3 & 0 & 0 & 0 \\
\hline 56.98 & 57.30 & 1.6150 & 7 & 1 & 0 & 0 & 27 & 0 & 87.08 & 87.08 & 1.1182 & 10 & 1 & 1 & 1 & 1 & 2 \\
\hline 57.30 & & 1.6067 & 6 & 1 & 1 & 30 & & 59 & 87.14 & & 1.1176 & 4 & 3 & 1 & 0 & & 1 \\
\hline 59.29 & 59.30 & 1.5574 & 8 & 0 & 0 & 4 & 5 & 9 & & & & & & & & & \\
\hline 59.64 & 59.64 & 1.5491 & 3 & 2 & 1 & 27 & 24 & 57 & & & & & & & & & \\
\hline 60.29 & 60.28 & 1.5338 & 1 & 1 & 2 & 0 & 1 & 0 & & & & & & & & & \\
\hline 62.53 & 62.52 & 1.4842 & 4 & 0 & 2 & 0 & 0 & 0 & & & & & & & & & \\
\hline 64.25 & 64.26 & 1.4485 & 3 & 1 & 2 & 17 & 15 & 39 & & & & & & & & & \\
\hline
\end{tabular}


Table B.12. Simulated X-ray powder pattern for $\mathrm{Ca}_{0.9} \mathrm{Sr}_{1,1} \mathrm{CuO}_{3}$

\begin{tabular}{|c|c|c|c|c|c|c|c|c|c|c|c|c|c|c|c|c|c|}
\hline $2 \theta$ & PEAK & d & $\bar{n}$ & k & $\frac{1}{1}$ & NT) & $(\mathrm{PK})$ & (DS) & $2 \theta$ & JEAR & d & h & $\mathrm{k}$ & $\overline{l \mid \mathbb{N}}$ & VT) & PK & (DS) \\
\hline 14.18 & 14.20 & 6.241 & 2 & 0 & 0 & 23 & 28 & 8 & 66.10 & 66.10 & 1.4125 & 6 & 2 & $0 \quad 1$ & 111 & 10 & 27 \\
\hline 24.18 & 24.20 & 3.677 & 1 & 1 & 0 & 0 & 0 & 0 & 67.32 & 67.32 & 1.3898 & 5 & 2 & 1 & 6 & 5 & 14 \\
\hline 27.25 & 27.26 & 3.270 & 1 & 0 & 1 & 8 & 8 & 6 & 70.79 & 70.80 & 1.3299 & 8 & 1 & 1 & 7 & 6 & 19 \\
\hline 28.58 & 28.60 & 3.120 & 4 & 0 & 0 & 2 & 2 & 2 & 71.58 & 71.58 & 1.3172 & 5 & 1 & 2 & 3 & 3 & 9 \\
\hline 31.65 & 31.66 & 2.825 & 3 & 1 & 0 & 62 & 64 & 54 & 71.80 & 71.80 & 1.3137 & 6 & 0 & 2 & 8 & 8 & 22 \\
\hline 34.10 & 34.10 & 2.627 & 3 & 0 & 1 & 76 & 77 & 72 & 72.37 & 72.38 & 1.3047 & 9 & 1 & 0 & 3 & 3 & 8 \\
\hline 35.26 & 35.26 & 2.543 & 0 & 1 & 11 & 00 & 1001 & 100 & 73.76 & 73.76 & 1.2835 & 9 & 0 & 1 & 3 & 3 & 10 \\
\hline 38.18 & 38.18 & 2.355 & 2 & 1 & 1 & 2 & 2 & 2 & 74.57 & 74.58 & 1.2715 & 0 & 2 & 2 & 9 & 8 & 28 \\
\hline 43.16 & 43.16 & 2.094 & 5 & 1 & 0 & 8 & 8 & 10 & 76.22 & 76.38 & 1.2481 & 10 & 0 & 0 & 0 & 1 & 0 \\
\hline 43.47 & 43.46 & 2.080 & 6 & 0 & 0 & 25 & 24 & 33 & 76.38 & & 1.2459 & 2 & 2 & 2 & 1 & & 3 \\
\hline 45.07 & 45.08 & 2.010 & 5 & 0 & 1 & 11 & 11 & 16 & 77.87 & 77.86 & 1.2258 & 3 & 3 & 0 & 5 & 4 & 14 \\
\hline 46.00 & 46.00 & 1.9713 & 4 & 1 & 1 & 5 & 5 & 7 & 78.94 & 78.94 & 1.2118 & 8 & 2 & 0 & 3 & 3 & 10 \\
\hline 47.20 & 47.20 & 1.9241 & 0 & 2 & 0 & 25 & 24 & 37 & 79.90 & 79.90 & 1.1996 & 0 & 3 & 1 & 6 & 5 & 21 \\
\hline 49.54 & 49.54 & 1.8387 & 2 & 2 & 0 & 2 & 2 & 3 & 81.67 & 81.68 & 1.1781 & 2 & 3 & 1 & 0 & 0 & 1 \\
\hline 54.09 & 54.08 & 1.6942 & 0 & 0 & 2 & 15 & 14 & 28 & 81.71 & & 1.1775 & 4 & 2 & 2 & 0 & & 0 \\
\hline 55.36 & 55.36 & 1.6583 & 1 & 2 & 1 & 1 & 1 & 2 & 82.35 & 82.34 & 1.1701 & 7 & 1 & 2 & 0 & 0 & 1 \\
\hline 56.11 & 56.22 & 1.6377 & 4 & 2 & 0 & 0 & 2 & 1 & 84.32 & 84.32 & 1.1477 & 8 & 0 & 2 & 3 & 2 & 9 \\
\hline 56.21 & & 1.6350 & 2 & 0 & 2 & 1 & & 3 & 84.93 & 84.94 & 1.1409 & 5 & 3 & 0 & 1 & 1 & 3 \\
\hline 56.87 & 57.16 & 1.6178 & 7 & 1 & 0 & 0 & 27 & 1 & 86.44 & 86.44 & 1.1249 & 1 & 0 & 3 & 0 & 0 & 0 \\
\hline 57.16 & & 1.6102 & 6 & 1 & 1 & 30 & & 59 & 86.86 & 86.86 & 1.1205 & 10 & 1 & 1 & 1 & 1 & 3 \\
\hline 59.17 & 59.18 & 1.5602 & 8 & 0 & 0 & 4 & 5 & 9 & 86.93 & & 1.1197 & 4 & 3 & 1 & 0 & & 1 \\
\hline 59.50 & 59.50 & 1.5523 & 3 & 2 & 1 & 27 & 25 & 57 & 89.93 & 89.94 & 1.0900 & 3 & 0 & 3 & 4 & 3 & 15 \\
\hline 60.08 & 59.66 & 1.5388 & 1 & 1 & 2 & 0 & 15 & 0 & & & & & & & & & \\
\hline 62.31 & 62.32 & 1.4889 & 4 & 0 & 2 & 0 & 0 & 0 & & & & & & & & & \\
\hline 64.03 & 64.04 & 1.4530 & 3 & 1 & 2 & 17 & 15 & 40 & & & & & & & & & \\
\hline
\end{tabular}


Table B.13. Simulated $\mathrm{X}$-ray powder pattern for $\mathrm{Ca}_{0.8} \mathrm{Sr}_{1.2} \mathrm{CuO}_{3}$

\begin{tabular}{|c|c|c|c|c|c|c|c|c|c|c|c|c|c|c|c|c|c|}
\hline $2 \theta$ & PEAK & d & h & $\bar{k}$ & $\operatorname{ll|}$ & $\overline{\mid N T) \mid}$ & $(\mathrm{PK})$ & I(DS) & $2 \theta$ & PEAK & d & $h$ & $\mathrm{k}$ & & $\overline{\mid N T)}$ & $\overline{\mathrm{F})}$ & (DS) \\
\hline 14.15 & 14.16 & 6.252 & 2 & 0 & 0 & 21 & 26 & 8 & 65.97 & 65.96 & 1.4150 & 6 & 2 & 0 & 11 & 9 & 27 \\
\hline 24.14 & 24.16 & 3.684 & 1 & 1 & 0 & 0 & 0 & 0 & 67.16 & 67.16 & 1.3927 & 5 & 2 & 1 & 5 & 5 & 14 \\
\hline 27.16 & 27.16 & 3.281 & 1 & 0 & 1 & 7 & 7 & 5 & 70.63 & 70.62 & 1.3326 & 8 & 1 & 1 & 7 & 6 & 19 \\
\hline 28.53 & 28.54 & 3.126 & 4 & 0 & 0 & 2 & 3 & 2 & 71.35 & 71.36 & 1.3209 & 5 & 1 & 2 & 3 & 3 & 8 \\
\hline 31.59 & 31.60 & 2.830 & 3 & 1 & 0 & 63 & 65 & 55 & 71.57 & 71.56 & 1.3174 & 6 & 0 & 2 & 8 & 8 & 22 \\
\hline 34.00 & 34.00 & 2.635 & 3 & 0 & 1 & 76 & 78 & 73 & 72.22 & 72.22 & 1.3070 & 9 & 1 & 0 & 3 & 3 & 8 \\
\hline 35.17 & 35.18 & 2.550 & 0 & 1 & 1 & 00 & 100 & 100 & 73.59 & 73.58 & 1.2861 & 9 & 0 & 1 & 3 & 3 & 10 \\
\hline 38.08 & 38.08 & 2.361 & 2 & 1 & 1 & 1 & 2 & 2 & 74.34 & 74.34 & 1.2750 & 0 & 2 & 2 & 9 & 8 & 28 \\
\hline 43.08 & 43.08 & 2.098 & 5 & 1 & 0 & 7 & 8 & 10 & 76.06 & 76.14 & 1.2504 & 10 & 0 & 0 & 0 & 1 & 1 \\
\hline 43.39 & 43.38 & 2.084 & 6 & 0 & 0 & 24 & 24 & 33 & 76.14 & & 1.2493 & 2 & 2 & 2 & 1 & & 3 \\
\hline 44.96 & 44.96 & 2.015 & 5 & 0 & 1 & 11 & 11 & 16 & 77.71 & 77.72 & 1.2278 & 3 & 3 & 0 & 5 & 4 & 15 \\
\hline 45.89 & 45.90 & 1.9759 & 4 & 1 & 1 & 5 & 5 & 8 & 78.77 & 78.78 & 1.2140 & $y$ & 2 & 0 & 3 & 3 & 10 \\
\hline 47.12 & 47.12 & 1.9273 & 0 & 2 & 0 & 25 & 24 & 37 & 79.72 & 79.72 & 1.2019 & 0 & 3 & 1 & 6 & 5 & 21 \\
\hline 49.45 & 49.44 & 1.8418 & 2 & 2 & 0 & 2 & 2 & 3 & 81.46 & 81.48 & 1.1806 & 4 & 2 & 2 & 0 & 0 & 1 \\
\hline 53.88 & 53.88 & 1.7002 & 0 & 0 & 2 & 15 & 14 & 28 & 81.48 & & 1.1803 & 2 & 3 & 1 & 0 & & 1 \\
\hline 55.23 & 55.24 & 1.6618 & 1 & 2 & 1 & 1 & 1 & 2 & 82.09 & 82.08 & 1.1731 & 7 & 1 & 2 & 0 & 0 & 1 \\
\hline 56.01 & 56.00 & 1.6406 & 2 & 0 & 2 & 1 & 2 & 2 & 84.05 & 84.04 & 1.1507 & 8 & 0 & 2 & 3 & 2 & 9 \\
\hline 56.01 & & 1.6406 & 4 & 2 & 0 & 0 & & 1 & 84.76 & 84.76 & 1.1429 & 5 & 3 & 0 & 1 & 1 & 3 \\
\hline 56.76 & 57.02 & 1.6207 & 7 & 1 & 0 & 0 & 27 & 1 & 86.65 & 86.66 & 1.12271 & 10 & 1 & 1 & 1 & 1 & 3 \\
\hline 57.03 & & 1.6136 & 6 & 1 & 1 & 30 & & 59 & 86.73 & & 1.1219 & 4 & 3 & 1 & 0 & & 1 \\
\hline 59.05 & 59.06 & 1.5630 & 8 & 0 & 0 & 4 & 5 & 9 & 89.54 & 89.54 & 1.0937 & 3 & 0 & 3 & 4 & 3 & 15 \\
\hline 59.37 & 59.36 & 1.5556 & 3 & 2 & 1 & 27 & 25 & 58 & 89.90 & 89.90 & 1.0903 & 11 & 1 & 0 & 3 & 3 & 10 \\
\hline 59.87 & 59.52 & 1.5437 & 1 & 1 & 2 & 0 & 15 & 0 & & & & & & & & & \\
\hline 62.09 & 62.10 & 1.4936 & 4 & 0 & 2 & 0 & 0 & 1 & & & & & & & & & \\
\hline 63.82 & 63.82 & 1.4574 & 3 & 1 & 2 & 17 & 15 & 41 & & & & & & & & & \\
\hline
\end{tabular}


Table B.14. Simulated X-ray powder pattern for $\mathrm{Ca}\left(0,7 \mathrm{Sr}_{1,3} \mathrm{CuO}_{3}\right.$

\begin{tabular}{|c|c|c|c|c|c|c|c|c|c|c|c|c|c|c|c|c|}
\hline $2 \theta \quad F$ & PEAK & d & $\mathrm{h}$ & $k$ & e III & NT)i(l & PK) $\mid(\mid$ & (DS) & $2 \theta$ & PEAK & d & $h 1$ & $k \quad l \quad$ & |(INT) & PK)।(I & (DS) \\
\hline 14.13 & 14.14 & 6.283 & 2 & 0 & 02 & 2.0 & 24 & 7 & 67.01 & 67.00 & 1.3955 & 52 & 21 & 5 & 5 & 13 \\
\hline 24.10 & 24.12 & 3.690 & 1 & 1 & 0 & 0 & 0 & 0 & 70.46 & 70.48 & 1.3353 & 8 & 11 & 7 & 6 & 19 \\
\hline 27.06 & 27.06 & 3,292 & 1 & 0 & 1 & 6 & 6 & 4 & 71.12 & 71.12 & 1.3246 & 5 & 12 & 3 & 3 & 8 \\
\hline 28.48 & 28.48 & 3.132 & 4 & 0 & 0 & 3 & 3 & 2 & 71.33 & 71.34 & 1.3211 & $\theta$ & 02 & 8 & 8 & 22 \\
\hline 31.53 & 31.54 & 2.835 & 3 & 1 & $0 E$ & 63 & 66 & 55 & 72.07 & 72.08 & 1.3094 & 9 & 10 & 3 & 2 & 8 \\
\hline 33.90 & 33.90 & 2.642 & 3 & 0 & 17 & 767 & 77 & 73 & 73.41 & 73.42 & 1.2887 & 9 & 01 & 3 & 3 & 10 \\
\hline 35.07 & 35.08 & 2.557 & 0 & 1 & 110 & 001 & 001 & 00 & 74.10 & 74.10 & 1.2784 & 0 & 22 & 9 & 8 & 28 \\
\hline 37.98 & 37,98 & 2.367 & 2 & 1 & 1 & 1 & 1 & 1 & 75.89 & 75.90 & 1.25271 & 10 & 00 & 0 & 1 & 1 \\
\hline 43.00 & 43.00 & 2.102 & 5 & 1 & 0 & 7 & 8 & 9 & 75.90 & & 1.2526 & 2 & 22 & 1 & & 3 \\
\hline 43.30 & 43,30 & 2.088 & 6 & 0 & 0 & 24 & 2.4 & 32 & 77.56 & 77.56 & 1.2299 & 3 & 30 & 5 & 4 & 15 \\
\hline 44.85 & 44.84 & 2.019 & 5 & 0 & 1 & 11 & 10 & 15 & 77.93 & 77.78 & 1.2249 & 7 & 21 & 0 & 2 & 0 \\
\hline 45.78 & 45.78 & 1.9806 & 4 & 1 & 1 & 6 & 6 & 9 & 78.61 & 78.60 & 1.2161 & 8 & 20 & 3 & 3 & 10 \\
\hline 47.03 & 47.04 & 1.9305 & 0 & 2 & 0 & 25 & 23 & 37 & 79.53 & 79.54 & 1.2042 & 0 & $\begin{array}{ll}31 \\
\text { ( }\end{array}$ & 7 & 5 & 21 \\
\hline 49.36 & 49.36 & 1.8449 & 2 & 2 & 0 & 2 & 2 & 3 & 81.21 & 81.28 & 1.1836 & 4 & 22 & 0 & 0 & 1 \\
\hline 53.68 & 53.68 & 1.7061 & 0 & 0 & 2 & 15 & 14 & 28 & 81.29 & & 1.1826 & 2 & $\begin{array}{ll}3 & 1\end{array}$ & 0 & & 1 \\
\hline 55.10 & 55.10 & 1.6653 & 1 & 2 & 1 & 1 & 1 & 2 & 81.83 & 81.82 & 1.1762 & 7 & 12 & 0 & 0 & 1 \\
\hline 55.80 & 55.80 & 1.6461 & 2 & 0 & 2 & 1 & 1 & 2 & 83.78 & 83.78 & 1.1536 & 8 & 02 & 3 & 2 & 9 \\
\hline 55.90 & 55.92 & 1.6434 & 4 & 2. & 0 & 0 & 1 & 1 & 84.58 & 84.58 & 1.1448 & 5 & $\begin{array}{ll}30 \\
0\end{array}$ & 1 & 1 & 3 \\
\hline 56.65 & 56.90 & 1.6236 & 7 & 1 & 0 & 12 & 27 & 1 & 86.43 & 86.44 & 1.12491 & 10 & 11 & 1 & 1 & 3 \\
\hline 56.89 & & 1.6171 & 6 & 1 & 1 & 30 & & 59 & 86.52 & & 1.1240 & 4 & 31 & 0 & & 1 \\
\hline 58.94 & 58.94 & 1.5658 & 8 & 0 & 0 & 4 & 5 & 9 & 89.16 & 89.16 & 1.0974 & 3 & 03 & 4 & 3 & 15 \\
\hline 59.23 & 59.24 & 1.5588 & 3 & 2 & 1 & 27 & 24 & 58 & 89.70 & 89.90 & 1.09231 & 11 & 10 & 3 & 9 & 10 \\
\hline 61.88 & 61.88 & 1.4982 & 4 & 0 & 2 & 0 & 0 & 1 & 89.82 & & 1.0911 & 0 & 13 & 5 & & 18 \\
\hline 63.60 & 63.60 & 1.4618 & 3 & 1 & 2 & 17 & 15 & 41 & 89.91 & & 1.0903 & 6 & 22 & 8 & & 31 \\
\hline 65.84 & 65.84 & 1.4174 & 6 & 2 & 0 & 11 & 9 & 27 & & & & & & & & \\
\hline
\end{tabular}


Table B.15. Simulated $\mathrm{X}$-ray powder pattern for $\mathrm{Ca}\left(0,6 \mathrm{Sr}_{1,4} \mathrm{CuO}_{3}\right.$

\begin{tabular}{|c|c|c|c|c|c|c|c|c|c|c|c|c|c|c|c|c|c|}
\hline $2 \theta$ & PEAK & $d$ & $h$ & $k$ & l 11 & $(\mid N T) \mid$ & (PK) & $1(D S)$ & $2 \theta$ & PEAK & $d$ & $h$ & k & $\operatorname{l|l|}$ & NT)I & PK)I & (DS) \\
\hline 14.10 & 14.12 & 6.274 & 2 & 0 & 0 & 18 & 22 & 6 & 66.86 & 66.86 & 1.3983 & 5 & 2 & 1 & 5 & 4 & 13 \\
\hline 26.97 & 26.98 & 3.303 & 1 & 0 & 1 & 5 & 5 & 3 & 70.30 & 70,30 & 1.3380 & 8 & 1 & 1 & 7 & 6 & 19 \\
\hline 28.43 & 28.44 & 3.137 & 4 & 0 & 0 & 3 & 3 & 2 & 70.89 & 70.90 & 1.3283 & 5 & 1 & 2 & 3 & 3 & 8 \\
\hline 31.48 & 31.48 & 2.840 & 3 & 1 & 0 & 64 & 66 & 56 & 71.10 & 71.10 & 1.3248 & 6 & 0 & 2 & 8 & 8 & 23 \\
\hline 33.80 & 33.80 & 2.650 & 3 & 0 & 1 & 76 & 77 & 73 & 71.93 & 71.92 & 1.3117 & 9 & 1 & 0 & 3 & 2 & 8 \\
\hline 34.97 & 34.98 & 2.564 & 0 & 1 & 1 & 100 & 100 & 100 & 73.24 & 73.24 & 1.2914 & 9 & 0 & 1 & 3 & 3 & 10 \\
\hline 37.88 & 37.88 & 2.373 & 2 & 1 & 1 & 1 & 1 & 1 & 73.87 & 73.88 & 1.2819 & 0 & 2 & 2 & 10 & 8 & 28 \\
\hline 42.92 & 42.92 & 2.105 & 5 & 1 & 0 & 7 & 8 & 9 & 75.66 & 75.66 & 1.2559 & 2 & 2 & 2 & 1 & 1 & 2 \\
\hline 43.22 & 43.22 & 2.092 & 6 & 0 & 0 & 24 & 23 & 32 & 75.73 & & 1.2549 & 10 & 0 & 0 & 0 & & 1 \\
\hline 44.73 & 44.74 & 2.024 & 5 & 0 & 1 & 11 & 10 & 15 & 77.40 & 77.40 & 1.2320 & 3 & 3 & 0 & 5 & 4 & 15 \\
\hline 45.66 & 45.66 & 1.9852 & 4 & 1 & 1 & 6 & 6 & 9 & 77.75 & 77.62 & 1.2273 & 7 & 2 & 1 & 0 & 2 & 1 \\
\hline 46.95 & 46.96 & 1.9338 & 0 & 2 & 0 & 25 & 23 & 37 & 78.44 & 78.44 & 1.2182 & 8 & 2 & 0 & 3 & 3 & 10 \\
\hline 49.27 & 49.28 & 1.8480 & 2 & 2 & 0 & 2 & 1 & 3 & 79.35 & 79.36 & 1.2065 & 0 & 3 & 1 & 7 & 5 & 22 \\
\hline 53.48 & 53.48 & 1.7121 & 0 & 0 & 2 & 15 & 14 & 28 & 80.95 & 80.96 & 1.1866 & 4 & 2 & 2 & 0 & 0 & 1 \\
\hline 54.98 & 54.98 & 1.6689 & 1 & 2 & 1 & 1 & 1 & 1 & 81.11 & 81.10 & 1.1848 & 2 & 3 & 1 & 0 & 0 & 1 \\
\hline 55.60 & 55.60 & 1.6517 & 2 & 0 & 2 & 1 & 1 & 2 & 81.57 & 81.58 & 1.1792 & 7 & 1 & 2 & 0 & 0 & 1 \\
\hline 55.80 & 55.76 & 1.6462 & 4 & 2 & 0 & 1 & 1 & 1 & 83.52 & 83.52 & 1.1566 & 8 & 0 & 2 & 2 & 2 & 9 \\
\hline 56.54 & 56.76 & 1.6265 & 7 & 1 & 0 & 1 & 27 & 1 & 84.40 & 84.40 & 1.1467 & 5 & 3 & 0 & 1 & 1 & 3 \\
\hline 56.76 & & 1.6206 & 6 & 1 & 1 & 30 & & 59 & 86.22 & 86.22 & 1.1271 & 10 & 1 & 1 & 1 & 1 & 4 \\
\hline 58.03 & 58.02 & 1.5882 & 7 & 0 & 1 & 0 & 0 & 0 & 86.32 & & 1.1261 & 4 & 3 & 1 & 0 & & 2 \\
\hline 58.82 & 58.82 & 1.5686 & 8 & 0 & 0 & 4 & 5 & 9 & 88.78 & 88.78 & 1.1011 & 3 & 0 & 3 & 4 & 3 & 15 \\
\hline 59.10 & 59.10 & 1.5620 & 3 & 2 & 1 & 28 & 25 & 59 & 89.44 & 89.46 & 1.0947 & 0 & 1 & 3 & 5 & 6 & 18 \\
\hline 61.67 & 61.66 & 1.5029 & 4 & 0 & 2 & 0 & 0 & 1 & 89.49 & & 1.0942 & 11 & 1 & 0 & 3 & & 10 \\
\hline 63.39 & 63.38 & 1.4662 & 3 & 1 & 2 & 18 & 15 & 42 & 89.63 & 89.62 & 1.0929 & 6 & 2 & 2 & 8 & 8 & 31 \\
\hline 65.71 & 65.72 & 1.4199 & 6 & 2 & 0 & 11 & 9 & 27 & & & & & & & & & \\
\hline
\end{tabular}


Table B.16. Simulated $\mathrm{X}$-ray powder pattern for $\mathrm{Ca} 0.5 \mathrm{Sr}_{1.5} \mathrm{CuO}_{3}$

\begin{tabular}{|c|c|c|c|c|c|c|c|c|c|c|c|c|c|c|c|c|c|}
\hline $2 \theta$ & PEAK & d & h & k & e IIIN & NT)।(F & $(P K) \|$ & I(DS) & $2 \theta$ & PEAK & d & & k & $\ell \mid(\mid$ & NT)। & PK)I & (DS) \\
\hline 14.08 & 14.08 & 6.286 & 2 & 0 & 01 & 162 & 20 & $\theta$ & 65.68 & 65.58 & 1.4223 & $\theta$ & 2 & 0 & 11 & 9 & 27 \\
\hline 24.02 & 24.02 & 3.702 & 1 & 1 & 0 & 0 & 0 & 0 & 66.70 & 66.70 & 1.4011 & 5 & 2 & 1 & 5 & 4 & 13 \\
\hline 26.88 & 26.88 & 3.315 & 1 & 0 & 1 & 4 & 4 & 3 & 0.13 & 70.14 & 1.3408 & 8 & 1 & 1 & 7 & $\theta$ & 19 \\
\hline 28,37 & 28.38 & 3.143 & 4 & 0 & 0 & 3 & 4 & 0 & 70.66 & 70.66 & 1.3320 & 5 & 1 & 2 & 3 & 3 & 8 \\
\hline 31.42 & 31.42 & 2.845 & 3 & 1 & 06 & 65 & 65 & 56 & 70.87 & 70.88 & 1.3285 & 6 & 0 & 2 & 8 & 8 & 23 \\
\hline 33.70 & 33.72 & 2.657 & 3 & 0 & 17 & 77 & 76 & 73 & .78 & 71.78 & 1.3140 & 9 & 1 & 0 & 3 & 2 & 8 \\
\hline 34.87 & 34.88 & 2.571 & 0 & 1 & 110 & 0010 & 1001 & 100 & 3.07 & 73.06 & 1.2940 & 9 & 0 & 1 & 3 & 3 & 10 \\
\hline 37.78 & 37.78 & 2.379 & 2 & 1 & 1 & 1 & 1 & 1 & 3.64 & 73.64 & 1.2853 & 0 & 2 & 2 & 10 & 8 & 29 \\
\hline 42.84 & 42.84 & 2.109 & 5 & 1 & 0 & 7 & 7 & 9 & 5.43 & 75.42 & 1.2593 & 2 & 2 & 2 & 1 & 1 & 2 \\
\hline 43.14 & 43.14 & 2.095 & 6 & 0 & 02 & 24 & 23 & 32 & 75.58 & 75.62 & 1.25711 & 10 & 0 & 0 & 0 & 0 & 1 \\
\hline 44,62 & 62 & 2.02 & 5 & 0 & 11 & 10 & 10 & 14 & 25 & 4 & 41 & 3 & 3 & 0 & 5 & 4 & 15 \\
\hline 45.55 & 45.56 & 1.9898 & 4 & 1 & 1 & 7 & 7 & 10 & .57 & 77.48 & 1.2297 & 7 & 2 & 1 & 0 & 2 & 1 \\
\hline 46.87 & 46.86 & 1.9370 & 0 & 2 & 02 & 24 & 22 & 37 & 8.28 & 78.28 & 1.2203 & 8 & 2 & 0 & 3 & 3 & 10 \\
\hline 49.18 & 49.18 & 1.8511 & 2 & 2 & 0 & 1 & 1 & 2 & .17 & 79.18 & 1.2088 & 0 & 3 & 1 & 7 & 5 & 22 \\
\hline 53.28 & 53.28 & 1.7181 & 0 & 0 & 2. 1 & 16 & 14 & 29 & 80.70 & 80.70 & 1.1897 & 4 & 2 & 2 & 0 & 0 & 1 \\
\hline 4.85 & 1.86 & 1.6 & 1 & 2 & 1 & 1 & 1 & 1 & .92 & 80.92 & 70 & 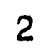 & 3 & 1 & 0 & 0 & \\
\hline 55.39 & 55.40 & 1.6573 & 2 & 0 & 2 & 1 & 1 & 2 & 1.32 & 81.32 & 1.1822 & 7 & 1 & 2 & 0 & 0 & 2 \\
\hline 55.70 & 55.70 & 1.6490 & 4 & 2 & 0 & 1 & 1 & 1 & 26 & 83.26 & & 8 & 0 & 2 & 2 & 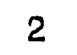 & 9 \\
\hline 56.43 & 56.62 & 1.6294 & 7 & 1 & 0 & 1 & 26 & 1 & 4.22 & 84.22 & 1.1487 & 5 & 3 & 0 & 1 & 1 & 3 \\
\hline 56.63 & & 1.6241 & 6 & 1 & 13 & 30 & & 60 & 86.01 & 86.02 & 1.12931 & 10 & 1 & 1 & 1 & 1 & 4 \\
\hline 57.89 & 57.90 & 1.5916 & 7 & 0 & 1 & 0 & 0 & 0 & 6.12 & & 1.1282 & 4 & 3 & 1 & 0 & & 2 \\
\hline 58.71 & 58.72 & 1.5714 & 8 & 0 & 0 & 4 & 5 & 0 & 3.41 & 88.40 & 1.1048 & 3 & 0 & 3 & 4 & 3 & 15 \\
\hline 58.96 & 58.96 & 1.5652 & 3 & 2 & 12 & 28 & 25 & 59 & \begin{tabular}{|l|}
89.06 \\
\end{tabular} & 89.06 & 1.0984 & 0 & 1 & 3 & 5 & 4 & 19 \\
\hline 61.46 & 61.46 & 1.5075 & 4 & 0 & 2 & 0 & 0 & 1 & 89.29 & 89.34 & 1.09621 & 11 & 1 & 0 & 3 & 9 & 10 \\
\hline 63.17 & 63.18 & 1.4706 & 3 & 1 & 21 & 18 & 15 & 42 & 89.35 & & 1.0956 & 6 & 2 & 2 & 8 & & 31 \\
\hline
\end{tabular}


Table B.17. Simulated X-ray powder pattern for $\mathrm{Ca}\left(0,4 \mathrm{Sr}_{1,6} \mathrm{CuO}_{3}\right.$

\begin{tabular}{|c|c|c|c|c|c|c|c|c|c|c|c|c|c|c|c|c|c|}
\hline $2 \theta$ & PEAK & $d$ & $\mathrm{~h}$ & $k$ & l|(II) & NTIK & $(\mathrm{PK}) \|$ & (DS) & $2 \theta$ & PEAK & d & & $k$ & & NT)। & PK)I & (DS) \\
\hline 14.05 & 14.06 & 6.297 & 2 & 0 & 0 & 15 & 18 & 5 & 65.45 & 65.46 & 1.4248 & 6 & 2 & 0 & 11 & 9 & 27 \\
\hline 23.98 & 23.98 & 3.708 & 1 & 1 & 0 & 0 & 0 & 0 & 63.55 & 66.56 & 1.4039 & 5 & 2 & 1 & 5 & 4 & 12 \\
\hline 26.79 & 25.80 & 3.326 & 1 & 0 & 1 & 3 & 4 & 2 & 69.97 & 69.98 & 1.3435 & 8 & 1 & 1 & 7 & 6 & 19 \\
\hline 28,32 & 28.34 & 3.148 & 4 & 0 & 0 & 4 & 4 & 3 & 70.44 & 70.44 & 1.3357 & 5 & 1 & 2 & 3 & 3 & 8 \\
\hline 31.37 & 31.38 & 2.850 & 3 & 1 & 0 & 65 & 66 & 57 & 70.65 & 70.64 & 1.3322 & 6 & 0 & 2 & 8 & $\theta$ & 23 \\
\hline 33.61 & 33.62 & 2.664 & 3 & 0 & 17 & 77 & 77 & 74 & 71.63 & 71.64 & 1.3164 & 9 & 1 & 0 & 3 & 2 & 8 \\
\hline 34.78 & 34.78 & 2.578 & 0 & 1 & 110 & 001 & 1001 & 100 & 72.89 & 72.90 & 1.2966 & 9 & 0 & 1 & 3 & 3 & 10 \\
\hline 37.68 & 37.68 & 2.385 & 2 & 1 & 1 & 1 & 1 & 1 & 73.41 & 73.42 & 1.2888 & 0 & 2 & 2 & 10 & 8 & 29 \\
\hline 42.77 & 42.78 & 2.113 & 5 & 1 & 0 & 6 & 7 & 9 & 75.19 & 75.20 & 1.2626 & 2 & 2 & 2 & 1 & 1 & 2 \\
\hline 43.06 & 43.06 & 2.099 & 6 & 0 & 0 & 24 & 23 & 32 & 75.42. & 75.42 & 1.25941 & 10 & 0 & 0 & 0 & 1 & 1 \\
\hline 44.51 & 44.52 & 2.034 & 5 & 0 & 1 & 10 & 9 & 14 & 77.09 & 77.10 & 1.2362 & 3 & 3 & 0 & 5 & 4 & 15 \\
\hline 45.44 & 45.44 & 1.9944 & 4 & 1 & 1 & 7 & 7 & 11 & 77.39 & 77.32 & 1.2321 & 7 & 2 & 1 & 0 & 2 & 1 \\
\hline 46.78 & 46.78 & 1.9403 & 0 & 2 & 0 & 24 & 23 & 37 & 78.12 & 78.12 & 1.2225 & 8 & 2 & 0 & 3 & 3 & 10 \\
\hline 49.09 & 49.10 & 1.8542 & 2 & 2 & 0 & 1 & 1 & 2 & 78.99 & 79.00 & 1.2111 & 0 & 3 & 1 & 7 & 5 & 22 \\
\hline 53.08 & 53.08 & 1.7240 & 0 & 0 & 2 & 16 & 14 & 29 & 80.46 & 80.46 & 1.1927 & 4 & 2 & 2 & 0 & 0 & 1 \\
\hline 54.73 & 54.72 & 1.6759 & 1 & 2 & 1 & 0 & 0 & 1 & 80.74 & 80.72 & 1.1893 & 2 & 3 & 1 & 0 & 0 & 1 \\
\hline 55.19 & 55.20 & 1.6628 & 2 & 0 & 2 & 1 & 1 & 2 & 81.07 & 81.06 & 1.1853 & 7 & 1 & 2 & 1 & 0 & 2 \\
\hline 55.59 & 55.60 & 1.6518 & 4 & 2 & 0 & 1 & 1 & 1 & 83.00 & 83.00 & 1.1625 & 8 & 0 & 2 & 2 & 2 & 9 \\
\hline 56.32 & 56.50 & 1.6322 & 7 & 1 & 0 & 1 & 27 & 2 & 84.05 & 84.04 & 1.1506 & 5 & 3 & 0 & 1 & 1 & 3 \\
\hline 56.49 & & 1.6276 & 6 & 1 & 1 & 30 & & 59 & 85.80 & 85.80 & 1.1315 & 10 & 1 & 1 & 1 & 1 & 4 \\
\hline 57.75 & 57.76 & 1.5951 & 7 & 0 & 1 & 0 & 0 & 1 & 85.92 & & 1.1303 & 4 & 3 & 1 & 1 & & 2 \\
\hline 58.59 & 58.60 & 1.5742 & 8 & 0 & 0 & 4 & 5 & 9 & 88.03 & 88.04 & 1.1085 & 3 & 0 & 3 & 4 & 3 & 15 \\
\hline 58.83 & 58.82 & 1.5685 & 3 & 2 & 1 & 28 & 25 & 59 & 88.69 & 88.70 & 1.1020 & 0 & 1 & 3 & 5 & 4 & 19 \\
\hline 61.25 & 61.24 & 1.5122 & 4 & 0 & 2 & 0 & 0 & 1 & 89.08 & 89.08 & 1.0983 & 6 & 2 & 2 & 8 & 9 & 31 \\
\hline 62.96 & 62.96 & 1.4751 & 3 & 1 & 2 & 18 & 16 & 43 & 89.09 & & 1.0981 & 11 & 1 & 0 & 3 & & 10 \\
\hline
\end{tabular}


Table B.18. Simulated $\mathrm{X}$-ray powder pattern for $\mathrm{Ca}_{0.3} \mathrm{Sr}_{1.7} \mathrm{CuO}_{3}$

\begin{tabular}{|c|c|c|c|c|c|c|c|c|c|c|c|c|c|c|c|c|c|}
\hline $2 \theta$ & PEAK & d & h & $k$ & $\ell \mid(\mathbb{I}$ & NTII & $(\mathrm{PK}) \|$ & (DS) & $2 \theta$ & PEAK & d & & k & l III & VTIII & ১K)! & (DS) \\
\hline $14 .(3$ & 14.04 & 6.308 & 2 & 0 & 0 & 14 & 17 & 5 & 65.33 & 65.32 & 1.4272 & 6 & 2 & 0 & 11 & 9 & 27 \\
\hline 23.94 & 23.94 & 3.715 & 1 & 1 & 0 & 0 & 0 & 0 & 66.40 & 66.40 & 1.4067 & 5 & 2 & 1 & 5 & 4 & 12 \\
\hline 26.69 & 26.70 & 3.337 & 1 & 0 & 1 & 3 & 3 & 2 & 69.81 & 69.80 & 1.3462 & 8 & 1 & 1 & 7 & 6 & 19 \\
\hline 28.27 & 28.28 & 3.154 & 4 & 0 & 0 & 4 & 4 & 3 & 70.21 & 70.22 & 1.3394 & 5 & 1 & 2 & 3 & 3 & 8 \\
\hline 31.31 & 31.32 & 2.854 & 3 & 1 & 0 & 65 & 67 & 57 & 70.42 & 70.42 & 1.3359 & 6 & 0 & 2 & 8 & 8 & 23 \\
\hline 33.51 & 33.52 & 2.672 & 3 & 0 & 1 & 77 & 79 & 74 & 71.48 & 71.48 & 1.3187 & 9 & 1 & 0 & 3 & 2 & 8 \\
\hline 34.68 & 34.68 & 2.584 & 0 & 1 & 111 & 001 & 1001 & 100 & 72.72 & 72.72 & 1.2992 & 9 & 0 & 1 & 3 & 3 & 10 \\
\hline 37.58 & 37.58 & 2.391 & 2 & 1 & 1 & 1 & 1 & 1 & 73.18 & 73.18 & 1.2922 & 0 & 2 & 21 & 10 & 8 & 29 \\
\hline 42.69 & 42.70 & 2.116 & 5 & 1 & 0 & 6 & 7 & 8 & 74.96 & 74.96 & 1.2659 & 2 & 2 & 2 & 1 & 1 & 2 \\
\hline 42.98 & 42.98 & 2.103 & 6 & 0 & 0 & 23 & 23 & 32 & 75.26 & 75.20 & 1.26161 & 10 & 0 & 0 & 0 & 0 & 1 \\
\hline 44.40 & 44.40 & 2.039 & 5 & 0 & 1 & 10 & 9 & 14 & 76.94 & 76.94 & 1.2382 & 3 & 3 & 0 & 5 & 4 & 16 \\
\hline 45.33 & 45.34 & 1.9990 & 4 & 1 & 1 & 8 & 8 & 12 & 77.21 & 77.16 & 1.2345 & 7 & 2 & 1 & 0 & 2 & 1 \\
\hline 46.70 & 46.70 & 1.9435 & 0 & 2 & 0 & 24 & 23 & 37 & 77.96 & 77.96 & 1.2246 & 8 & 2 & 0 & 3 & 2 & 10 \\
\hline 49.00 & 49.00 & 1.8573 & 2 & 2 & 0 & 1 & 1 & 2 & 78.82 & 78.82 & 1.2134 & 0 & 3 & 1 & 7 & 5 & 22 \\
\hline 52.88 & 52.88 & 1.7300 & 0 & 0 & 2 & 16 & 14 & 29 & 80.21 & 80.22 & 1.1957 & 4 & 2 & 2 & 0 & 0 & 1 \\
\hline 54.60 & 54.60 & 1.6794 & 1 & 2 & 1 & 0 & 0 & 1 & 80.55 & 80.54 & 1.1915 & 2 & 3 & 1 & 0 & 0 & 1 \\
\hline 54.99 & 55.00 & 1.6684 & 2 & 0 & 2 & 1 & 1 & 2 & 80.82 & 80.82 & 1.1883 & 7 & 1 & 2 & 1 & 1 & 2 \\
\hline 55.49 & 55.50 & 1.6546 & 4 & 2 & 0 & 1 & 1 & 2 & 82.74 & 82.74 & 1.1655 & 8 & 0 & 2 & 2 & 2 & 9 \\
\hline 56.21 & 56.36 & 1.6351 & 7 & 1 & 0 & 1 & 27 & 2 & 83.87 & 83.88 & 1.1526 & 5 & 3 & 0 & 1 & 1 & 3 \\
\hline 56.36 & & 1.6311 & 6 & 1 & 1 & 30 & & 60 & 85.60 & 85.60 & 1.13381 & 10 & 1 & 1 & 1 & 1 & 5 \\
\hline 57.62 & 57.62 & 1.5985 & 7 & 0 & 1 & 0 & 1 & 1 & 85.72 & 85.70 & 1.1325 & 4 & 3 & 1 & 1 & 1 & 2 \\
\hline 58.48 & 58.50 & 1.5771 & 8 & 0 & 0 & 4 & 6 & 9 & 87.67 & 87.66 & 1.1122 & 3 & 0 & 3 & 4 & 3 & 15 \\
\hline 58.70 & 58.70 & 1.5717 & 3 & 2 & 1 & 28 & 26 & 60 & 88.32 & 88.32 & 1.1057 & 0 & 1 & 3 & 5 & 4 & 19 \\
\hline 61.04 & 61.04 & 1.5168 & 4 & 0 & 2 & 1 & 1 & 1 & 88.80 & 88.80 & 1.1009 & 6 & 2 & 2 & 8 & 7 & 31 \\
\hline 62.75 & 62.76 & 1.479 .5 & 3 & 1 & 2 & 18 & 16 & 43 & 88.89 & & 1.1001 & 11 & 1 & 0 & 3 & & 10 \\
\hline
\end{tabular}


Table B.19. Simulated X-ray powder pattern for $\mathrm{Ca}_{0.2} \mathrm{Sr}_{1.8} \mathrm{CuO}_{3}$

\begin{tabular}{|c|c|c|c|c|c|c|c|c|c|c|c|c|c|c|c|c|c|}
\hline \multirow{2}{*}{$\frac{2 \theta}{13.98}$} & \multirow{2}{*}{$\frac{\text { PEAK }}{13.98}$} & \multirow{2}{*}{$\frac{d}{6.331}$} & & \multirow{2}{*}{$\frac{k}{0}$} & \multicolumn{4}{|c|}{ l I(INT)|(PK)|(DS) } & \multirow{2}{*}{$2 \theta$} & \multirow{2}{*}{$\frac{\text { PEAK }}{71.22}$} & \multirow{2}{*}{$\frac{d}{1.3231}$} & & \multirow{2}{*}{$\frac{k}{1}$} & \multicolumn{4}{|c|}{$\ell|(\mid N T)|(P K) \mid(D S)$} \\
\hline & & & & & 0 & 13 & 15 & 4 & & & & & & 0 & 3 & 2 & 8 \\
\hline 23.89 & 23.90 & 3.721 & 1 & 1 & 0 & 0 & 1 & 0 & 72.43 & 72.42 & 1.3039 & 9 & 0 & 1 & 3 & 3 & 10 \\
\hline 26.60 & 26.60 & 3.348 & 1 & 0 & 1 & 2 & 2 & 2 & 72.96 & 72.96 & 1.2956 & 0 & 2 & 2 & 10 & 8 & 29 \\
\hline 28.17 & 28.18 & 3.165 & 4 & 0 & 0 & 5 & 5 & 3 & 74.73 & 74.72 & 1.2693 & 2 & 2 & 2 & 1 & 1 & 2 \\
\hline 31.23 & 31.24 & 2.862 & 3 & 1 & 0 & 66 & 68 & 58 & 74.94 & 74.94 & 1.26611 & 10 & 0 & 0 & 0 & 1 & 1 \\
\hline 33.39 & 33.40 & 2.681 & 3 & 0 & 1 & 78 & 79 & 74 & 76.77 & 76.78 & 1.2405 & 3 & 3 & 0 & 5 & 4 & 16 \\
\hline 34.59 & 34.60 & 2.591 & 0 & 1 & 11 & 1001 & $100 \cdot 1$ & 100 & 76.96 & 77.00 & 1.2380 & 7 & 2 & 1 & 0 & 3 & 1 \\
\hline 37.47 & 37.48 & 2.398 & 2 & 1 & 1 & 1 & 1 & 1 & 77.70 & 77.70 & 1.2280 & 8 & 2 & 0 & 3 & 2 & 10 \\
\hline 42.55 & 42.56 & 2.123 & 5 & 1 & 0 & 6 & 7 & 8 & 78.64 & 78.64 & 1.2157 & 0 & 3 & 1 & 7 & 6 & 22 \\
\hline 42.82 & 42.82 & 2.110 & 6 & 0 & 0 & 23 & 23 & 32 & 79.94 & 79.94 & 1.1991 & 4 & 2 & 2 & 0 & 0 & 2 \\
\hline 44.23 & 44.24 & 2.046 & 5 & 0 & 1 & 9 & 9 & 13 & 80.36 & 80.50 & i. 1938 & 2 & 3 & 1 & 0 & 1 & 1 \\
\hline 45.18 & 45.18 & 2.005 & 4 & 1 & 1 & 8 & 8 & 12 & 80.49 & & 1.1923 & 7 & 1 & 2 & 1 & & 2 \\
\hline 46.62 & 46.62 & 1.9467 & 0 & 2 & 0 & 24 & 23 & 37 & 82.39 & 82.40 & 1.1696 & 8 & 0 & 2 & 2 & 2 & 9 \\
\hline 48.91 & 48.92 & 1.8607 & 2 & 2 & 0 & 1 & 1 & 2 & 83.66 & 83.66 & 1.1550 & 5 & 3 & 0 & 1 & 1 & 3 \\
\hline 52.69 & 52.68 & 1.7359 & 0 & 0 & 2 & 16 & 14 & 29 & 85.24 & 85.24 & 1.13761 & 10 & 1 & 1 & 1 & 1 & 5 \\
\hline 54.48 & 54.48 & 1.6829 & 1 & 2 & 1 & 0 & 0 & 1 & 85.50 & 85.50 & 1.1348 & 4 & 3 & 1 & 1 & 1 & 3 \\
\hline 54.79 & 54.80 & 1.6741 & 2 & 0 & 2 & 1 & 1 & 2 & 87.29 & 87.28 & 1.1161 & 3 & 0 & 3 & 4 & 3 & 16 \\
\hline 55.36 & 55.36 & 1.6582 & 4 & 2 & 0 & 1 & 1 & 2 & 87.96 & 87.96 & 1.1093 & 0 & 1 & 3 & 5 & 4 & 19 \\
\hline 56.01 & 56.16 & 1.6404 & 7 & 1 & 0 & 1 & 27 & 2 & 88.48 & 88.48 & 1.1041 & 6 & 2 & 2 & 8 & 8 & 31 \\
\hline 56.17 & & 1.6363 & 6 & 1 & 1 & 30 & & 60 & 88.51 & & 1.10381 & 11 & 1 & 0 & 3 & & 10 \\
\hline 57.40 & 57.40 & 1.6041 & 7 & 0 & 1 & 0 & 1 & 1 & 89.65 & 89.66 & 1.0927 & 2 & 1 & 3 & 0 & 3 & 1 \\
\hline 58.25 & 58.26 & 1.5827 & 8 & 0 & 0 & 4 & 5 & 8 & 89.67 & & 1.09261 & 11 & 0 & 1 & 3 & & 12 \\
\hline 58.55 & 58.54 & 1.5753 & 3 & 2 & 1 & 28 & 25 & 60 & & & & & & & & & \\
\hline 60.81 & 60.80 & 1.5221 & 4 & 0 & 2 & 1 & 1 & 1 & & & & & & & & & \\
\hline 62.53 & 62.54 & 1.4842 & 3 & 1 & 2 & 18 & 16 & 44 & & & & & & & & & \\
\hline 65.14 & 65.14 & 1.4309 & 6 & 2 & 0 & 11 & 9 & 27 & & & & & & & & & \\
\hline 66.21 & 66.22 & 1.4103 & 5 & 2 & 1 & 5 & 4 & 12 & & & & & & & & & \\
\hline 69.54 & 69.54 & 1.3507 & 8 & 1 & 1 & 7 & 6 & 19 & & & & & & & & & \\
\hline 69.95 & 69.96 & 1.3438 & 5 & 1 & 2 & 3 & 3 & 7 & & & & & & & & & \\
\hline 70.14 & 70.14 & 1.3406 & 6 & 0 & 2 & 8 & 8 & 23 & & & & & & & & & \\
\hline
\end{tabular}


Table B.20. Simulated X-ray powder pattern for $\mathrm{Ca}\left(0.1 \mathrm{Sr}_{1,9} \mathrm{CuO}_{3}\right.$

\begin{tabular}{|c|c|c|c|c|c|c|c|c|c|c|c|c|c|c|c|c|c|}
\hline $2 \theta$ & PEAK & $d$ & $h$ & $k$ & $\operatorname{ll} 11$ & $|N T|$ & $|(P K)|$ & I(DS) & $2 \theta$ & PEAK & $d$ & $h$ & $k$ & 11 & $|N T|$ & PK) & (DS) \\
\hline 13.98 & 13.98 & 6.331 & 2 & 0 & 0 & 12 & 14 & 4 & 71.19 & 71.20 & 1.3234 & 9 & 1 & 0 & 3 & 2 & 8 \\
\hline 23.85 & 23.86 & 3.727 & 1 & 1 & 0 & 1 & 1 & 0 & 72.39 & 72.38 & 1.3045 & 9 & 0 & 1 & 3 & 3 & 10 \\
\hline 26.51 & 26.52 & 3.359 & 1 & 0 & 1 & 2 & 2 & 1 & 72.74 & 72.74 & 1.2991 & 0 & 2 & 2 & 10 & 8 & 29 \\
\hline 28.17 & 28.18 & 3.165 & 4 & 0 & 0 & 5 & 5 & 4 & 74.50 & 74.50 & 1.2725 & 2 & 2 & 2 & 0 & 0 & 2 \\
\hline 31.20 & 31.20 & 2.864 & 3 & 1 & 0 & 66 & 67 & 58 & 74.94 & 74.94 & 1.2661 & 10 & 0 & 0 & 0 & 0 & 1 \\
\hline 33.32 & 33.32 & 2.687 & 3 & 0 & 1 & 78 & 78 & 74 & 76.64 & 76.64 & 1.2424 & 3 & 3 & 0 & 5 & 4 & 16 \\
\hline 34.49 & 34.50 & 2.598 & 0 & 1 & 11 & 100 & 100 & 100 & 76.86 & 76.86 & 1.2394 & 7 & 2 & 1 & 0 & 3 & 1 \\
\hline 37.38 & 37.38 & 2.404 & 2 & 1 & 1 & 0 & 0 & 0 & 77.64 & 77.64 & 1.2289 & 8 & 2 & 0 & 3 & 2 & 9 \\
\hline 42.53 & 42.54 & 2.124 & 5 & 1 & 0 & 6 & 7 & 8 & 78.46 & 78.46 & 1.2179 & 0 & 3 & 1 & 7 & 5 & 22 \\
\hline 42.82 & 42.82 & 2.110 & 6 & 0 & 0 & 23 & 22 & 31 & 79.73 & 79.72 & 1.2018 & 4 & 2 & 2 & 1 & 0 & 2 \\
\hline 44.18 & 44.18 & 2.048 & 5 & 0 & 1 & 9 & 9 & 13 & 80.19 & 80.32 & 1.1960 & 2 & 3 & 1 & 0 & 1 & 1 \\
\hline 45.11 & 45.12 & 2.008 & 4 & 1 & 1 & 9 & 8 & 13 & 80.32 & & 1.1944 & 7 & 1 & 2 & 1 & & 3 \\
\hline 46.54 & 46.54 & 1.9500 & 0 & 2 & 0 & 24 & 22 & 37 & 82.23 & 82.24 & 1.1714 & 8 & 0 & 2 & 2 & 2 & 9 \\
\hline 48.83 & 48.84 & 1.8636 & 2 & 2 & 0 & 1 & 1 & 2 & 83.53 & 83.52 & 1.1565 & 5 & 3 & 0 & 1 & 1 & 3 \\
\hline 52.49 & 52.50 & 1.7419 & 0 & 0 & 2 & 16 & 14 & 29 & 85.18 & 85.18 & 1.1382 & 10 & 1 & 1 & 1 & 1 & 5 \\
\hline 54.36 & 54.36 & 1.6864 & 1 & 2 & 1 & 0 & 0 & 0 & 85.32 & 85.32 & 1.1367 & 4 & 3 & 1 & 1 & 1 & 3 \\
\hline 54.60 & 54.60 & 1.6795 & 2 & 0 & 2 & 1 & 1 & 1 & 86.94 & 86.94 & 1.1197 & 3 & 0 & 3 & 4 & 3 & 16 \\
\hline 55.29 & 55.30 & 1.6602 & 4 & 2 & 0 & 1 & 1 & 2 & 87.60 & 87.60 & 1.1130 & 0 & 1 & 3 & 5 & 4 & 19 \\
\hline 56.00 & 56.10 & 1.6409 & 7 & 1 & 0 & 1 & 27 & 2 & 88.26 & 88.26 & 1.1063 & $\hat{\jmath}$ & 2 & 2 & 8 & 6 & 31 \\
\hline 56.10 & & 1.6380 & 6 & 1 & 1 & 29 & & 60 & 88.49 & 88.52 & 1.1040 & 11 & 1 & 0 & 3 & 5 & 10 \\
\hline 57.35 & 57.36 & 1.6053 & 7 & 0 & 1 & 0 & 1 & 1 & 89.29 & 89.30 & 1.0962 & 2 & 1 & 3 & 0 & 0 & 1 \\
\hline 58,25 & 58.44 & 1.5827 & 8 & 0 & 0 & 4 & 26 & 8 & 89.63 & 89.62 & 1.0929 & 11 & 0 & 1 & 3 & 2 & 12 \\
\hline 58.43 & & 1.5781 & 3 & 2 & 1 & 28 & & 61 & & & & & & & & & \\
\hline 60.63 & 60.64 & 1.5261 & 4 & 0 & 2 & 1 & 1 & 2 & & & & & & & & & \\
\hline 62.34 & 62.34 & 1.4883 & 3 & 1 & 2 & 19 & 16 & 44 & & & & & & & & & \\
\hline 65.08 & 65.08 & 1.4321 & 6 & 2 & 0 & 10 & 9 & 27 & & & & & & & & & \\
\hline 66.11 & 66.10 & 1.4123 & 5 & 2 & 1 & 4 & 4 & 12 & & & & & & & & & \\
\hline 69.49 & 69.48 & 1.3516 & 8 & 1 & 1 & 7 & 6 & 18 & & & & & & & & & \\
\hline 69.77 & 69.70 & 1.3468 & 5 & 1 & 2 & 3 & 4 & 7 & & & & & & & & & \\
\hline 69.98 & 69.98 & 1.3434 & 6 & 0 & 2 & 8 & 8 & 23 & & & & & & & & & \\
\hline
\end{tabular}


Table B.21. Simulated X-ray powder pattern for $\mathrm{Sr}_{2} \mathrm{CuO}_{3}$

\begin{tabular}{|c|c|c|c|c|c|c|c|c|c|c|c|c|c|c|c|c|c|}
\hline \multirow{2}{*}{$\frac{2 \theta}{13.95}$} & \multirow{2}{*}{$\frac{\text { PEAK }}{13.96}$} & \multirow{2}{*}{$\frac{d}{6.342}$} & \multirow{2}{*}{$\frac{h}{2}$} & \multirow{2}{*}{$\frac{k}{0}$} & \multicolumn{4}{|c|}{$\ell|(I N T)|(P K) \mid(D S)$} & \multirow{2}{*}{\begin{tabular}{|l}
$2 \theta$ \\
65.96
\end{tabular}} & \multirow{2}{*}{$\frac{\text { PEAK }}{65.96}$} & \multirow{2}{*}{$\frac{d}{1.4151}$} & & \multirow{2}{*}{$\frac{k}{2}$} & \multicolumn{4}{|c|}{$\ell|(I N T)|(P K) \mid(D S)$} \\
\hline & & & & & 0 & 3 & 3 & 1 & & & & & & 1 & 3 & 2 & 7 \\
\hline 23.81 & 23.82 & 3.733 & 1 & 1 & 0 & 7 & 7 & 4 & 69.33 & 69.32 & 1.3544 & 8 & 1 & 1 & 5 & 4 & 14 \\
\hline 28.12 & 28.12 & 3.171 & 4 & 0 & 0 & 12 & 13 & 9 & 69.55 & 69.54 & 1.3505 & 5 & 1 & 2 & 1 & 4 & 4 \\
\hline 31.15 & 31.16 & 2.869 & 3 & 1 & 0 & 62 & 63 & 54 & 69.76 & 69.76 & 1.3471 & 6 & 0 & 2 & 7 & 7 & 21 \\
\hline 33.23 & 33.24 & 2.694 & 3 & 0 & 1 & 76 & 76 & 72 & 71.05 & 71.06 & 1.3257 & 9 & 1 & 0 & 2 & 2 & 6 \\
\hline 34.40 & 34.40 & 2.605 & 0 & 1 & 11 & 100 & 100 & 100 & 72.22 & 72.22 & 1.3071 & 9 & 0 & 1 & 3 & 3 & 8 \\
\hline 37.29 & 37.30 & 2.410 & 2. & 1 & 1 & 0 & 1 & 1 & 72.51 & 72.52 & 1.3025 & 0 & 2 & 2 & 9 & 8 & 28 \\
\hline 42.45 & 42.46 & 2.128 & 5 & 1 & 0 & 3 & 4 & 5 & 72.98 & 72.72 & 1.2953 & 1 & 3 & 0 & 0 & 4 & 1 \\
\hline 42.74 & 42.74 & 2.114 & 6 & 0 & 0 & 22 & 22 & 30 & 74.79 & 74.78 & 1.2684 & 10 & 0 & 0 & 1 & 1 & 3 \\
\hline 44.07 & 44.08 & 2.053 & 5 & 0 & 1 & 6 & 6 & 9 & 76.48 & 76.48 & 1.2445 & 3 & 3 & 0 & 4 & 4 & 14 \\
\hline 45.00 & 45.00 & 2.013 & 4 & 1 & 1 & 20 & 19 & 29 & 76.68 & 76.70 & 1.2418 & 7 & 2 & 1 & 2 & 3 & 6 \\
\hline 46.45 & 46.46 & 1.9532 & 0 & 2 & 0 & 24 & 22 & 37 & 77.48 & 77.48 & 1.2310 & 8 & 2 & 0 & 2 & 2 & 7 \\
\hline 48.74 & 48.74 & 1.8667 & 2 & 2 & 0 & 0 & 0 & 0 & 78.29 & 78.28 & 1.2202 & 0 & 3 & 1 & 6 & 5 & 21 \\
\hline 52.30 & 52.30 & 1.7478 & 0 & 0 & 2 & 16 & 14 & 29 & 79.49 & 79.48 & 1.2048 & 4 & 2 & 2 & 2 & 2 & 6 \\
\hline 54.24 & 54.24 & 1.6899 & 1 & 2 & 1 & 0 & 0 & 0 & 80.08 & 80.08 & 1.1974 & 7 & 1 & 2 & 2 & 2 & 8 \\
\hline 55.19 & 55.18 & 1.6630 & 4 & 2 & 0 & 3 & 3 & 6 & 81.98 & 81.98 & 1.1743 & 8 & 0 & 2 & 2 & 1 & 7 \\
\hline 55.89 & 55.98 & 1.6438 & 7 & 1 & 0 & 3 & 26 & 7 & 83.36 & 83.36 & 1.1584 & 5 & 3 & 0 & 0 & 0 & 1 \\
\hline 55.97 & & 1.6415 & 6 & 1 & 1 & 27 & & 55 & 84.98 & 84.98 & 1.1404 & 10 & 1 & 1 & 3 & 3 & 13 \\
\hline 57.22 & 57.22 & 1.6087 & 7 & 0 & 1 & 2 & 2 & 4 & 85.13 & 85.12 & 1.1388 & 4 & 3 & 1 & 2 & 2 & 8 \\
\hline 58.14 & 58.30 & 1.5855 & 8 & 0 & 0 & 3 & 25 & 7 & 86.58 & 86.58 & 1.1234 & 3 & 0 & 3 & 4 & 3 & 14 \\
\hline 58.24 & & 1.5830 & 1 & 1 & 2 & 1 & & 2 & 87.24 & 87.24 & 1.1166 & 0 & 1 & 3 & 4 & 4 & 17 \\
\hline 58.30 & & 1.5813 & 3 & 2 & 1 & 26 & & 57 & 88.00 & 88.00 & 1.1089 & 6 & 2 & 2 & 7 & 5 & 27 \\
\hline 60.43 & 60.42 & 1.5307 & 4 & 0 & 2 & 2 & 2 & 5 & 88.30 & 88.28 & 1.1059 & 11 & 1 & 0 & 2 & 4 & 8 \\
\hline 62.13 & 62.14 & 1.4927 & 3 & 1 & 2 & 17 & 15 & 40 & 89.41 & 89.40 & 1.0951 & 11 & 0 & 1 & 2 & 2 & 10 \\
\hline 64.95 & 64.96 & 1.4346 & 6 & 2 & 0 & 10 & 8 & 24 & & & & & & & & & \\
\hline
\end{tabular}


APPENDIX C: $(\mathrm{Ca}, \mathrm{Sr})_{14} \mathrm{Cu}_{24} \mathrm{O}_{41}$ 
Table C.1. Simulated X-ray powder pattern for $\mathrm{Sr}_{14} \mathrm{Cu}_{24} \mathrm{O}_{41}$

\begin{tabular}{|c|c|c|c|c|c|c|c|c|c|c|c|c|c|c|c|c|c|}
\hline \multirow{2}{*}{$\begin{array}{c}2 \theta \quad F \\
13.20\end{array}$} & \multirow{2}{*}{$\frac{\text { PEAK }}{13.22}$} & \multirow{2}{*}{$\frac{d}{6.700}$} & \multirow{2}{*}{$\frac{h}{0}$} & \multirow{2}{*}{$\frac{k}{2}$} & \multicolumn{4}{|c|}{$\ell|(\mid N T)|(P K) \mid(D S)$} & \multirow{2}{*}{$\frac{2 \theta}{47.99}$} & \multirow{2}{*}{ PEAK } & \multirow{2}{*}{$\frac{d}{1.8944}$} & \multirow{2}{*}{$\frac{h}{0}$} & \multicolumn{5}{|c|}{$l|(\mid N T)|(P K) \mid(D S)$} \\
\hline & & & & & 0 & 2 & 2 & 1 & & & & & 21 & 4 & 7 & & 11 \\
\hline 15.44 & 15.44 & 5.734 & 2 & 0 & 0 & 1 & 2 & 1 & 48.73 & 48.72 & 1.8674 & 2 & 01 & 4 & 3 & 3 & 6 \\
\hline 16.36 & 16.36 & 5.415 & 1 & 1 & 4 & 0 & 0 & 0 & 49.55 & 49.56 & 1.8382 & 6 & 2 & 0 & 11 & 10 & 20 \\
\hline 20.37 & 20.38 & 4.357 & 2 & 2 & 0 & 0 & 0 & 0 & 50.04 & 50.30 & 1.8214 & 1 & 7 & 4 & 0 & 17 & 0 \\
\hline 22.56 & 22.56 & 3.939 & 2 & 2 & 3 & 1 & 1 & 1 & 50.29 & & 1.8129 & 5 & 3 & 72 & 20 & & 37 \\
\hline 24.73 & 24.74 & 3.598 & 1 & 1 & 7 & 9 & 9 & 7 & 50.71 & 50.70 & 1.7988 & 2 & 21 & 4 & 3 & 3 & 6 \\
\hline 24.95 & & 3.566 & 1 & 3 & 4 & 0 & & 0 & 51.85 & 51.86 & 1.7619 & 4 & 6 & 0 & 1 & 1 & 1 \\
\hline 26.59 & 26.60 & 3.350 & 0 & 4 & 0 & 15 & 15 & 13 & 52.22 & 52.22 & 1.7503 & 5 & 110 & 0 & 4 & 4 & 9 \\
\hline 30.89 & 30.90 & 2.893 & 2 & 4 & 01 & 1001 & 100 & 100 & 53.25 & 53.26 & 1.7189 & 3 & 510 & 0 & 3 & 3 & 6 \\
\hline 31.17 & 31.20 & 2.867 & 4 & 0 & 0 & 10 & 85 & 10 & 53.77 & 53.76 & 1.7035 & 1 & 77 & 7 & 4 & 4 & 8 \\
\hline 31.19 & & 2.865 & 1 & 3 & 7 & 73 & & 74 & 54.76 & 54.76 & 1.6750 & 0 & 8 & 01 & 14 & 12 & 29 \\
\hline 32.41 & 32.42 & 2.760 & 2 & 4 & 3 & 0 & 1 & 0 & 55.29 & 55.28 & 1.6602 & 6 & 4 & 0 & 1 & 1 & 2 \\
\hline 33.27 & 33.28 & 2.691 & 3 & 1 & 7 & 59 & 56 & 65 & 55.96 & 55.96 & 1.6418 & 5 & 31 & 0 & 2 & 2 & 5 \\
\hline 33.98 & 33.98 & 2.636 & 4 & 2 & 0 & 10 & 13 & 12 & 56.36 & 56.36 & 1.6311 & 2 & 41 & 41 & 15 & 13 & 34 \\
\hline 33.99 & & 2.635 & 1 & 11 & 10 & 3 & & 3 & 56.54 & 56.52 & 1.6265 & 4 & 01 & 4 & 2 & 9 & 4 \\
\hline 36.78 & 36.78 & 2.441 & 1 & 5 & 4 & 0 & 0 & 0 & 57.25 & 57.26 & 1.6078 & 2 & 8 & 0 & 0 & 0 & 0 \\
\hline 38.44 & 38.44 & 2.340 & 3 & 3 & 7 & 6 & 5 & 7 & 57.78 & 57.78 & 1.5944 & 5 & 5 & 7 & 6 & 5 & 13 \\
\hline 39.08 & 39.08 & 2.303 & 1 & 31 & 10 & 3 & 3 & 4 & 58.33 & 58.34 & 1.5806 & 4 & 21 & 4 & 2 & 2 & 4 \\
\hline 40.35 & 40.36 & 2.233 & 0 & 6 & 0 & 3 & 3 & 4 & 58.74 & 58.74 & 1.5706 & 3 & 7 & 7 & 14 & 12 & 33 \\
\hline 40.80 & 40.80 & 2.210 & 3 & 11 & 10 & 9 & 9 & 13 & 59.21 & 59.20 & 1.5593 & 1 & 71 & 10 & 1 & 1 & 3 \\
\hline 41.42 & 41.44 & 2.178 & 4 & 4 & 0 & 15 & 33 & 22 & 61.62 & 61.62 & 1.5038 & 7 & 1 & 7 & 1 & 1 & 2 \\
\hline 41.44 & & 2.177 & 1 & 5 & 7 & 22 & & 31 & 62.75 & 62.76 & 1.4795 & 0 & 61 & 14 & 5 & 4 & 13 \\
\hline 43.45 & 43.46 & 2.081 & 2 & 6 & 0 & 2 & 2 & 3 & 63.00 & 62.94 & 1.4743 & 5 & 51 & 10 & 2 & 3 & 4 \\
\hline 44.60 & 44.60 & 2.030 & 2 & 6 & 3 & 0 & 0 & 1 & 63.53 & 63.54 & 1.4631 & 4 & 41 & 141 & 11 & 9 & 28 \\
\hline 45.24 & 45.24 & 2.003 & 3 & 3 & 10 & 6 & 6 & 10 & 63.91 & 64.08 & 1.4554 & 3 & 71 & 10 & 1 & 6 & 3 \\
\hline 45.91 & 45.92 & 1.9750 & 0 & 01 & 14 & 17 & 16 & 29 & 64.07 & & 1.4522 & 6 & 6 & 0 & 7 & & 19 \\
\hline 46.23 & 46.22 & 1.9622 & 5 & 1 & 7 & 2 & 3 & 3 & & & & & & & & & \\
\hline 46.36 & & 1.9570 & 5 & 3 & 4 & 0 & & 0 & & & & & & & & & \\
\hline 47.53 & 47.54 & 1.9115 & 6 & 0 & 0 & 10 & 9 & 18 & & & & & & & & & \\
\hline 47.89 & 47.98 & 1.8979 & 1 & 5 & 10 & 1 & 6 & 1 & & & & & & & & & \\
\hline
\end{tabular}


Table C.2. Simulated X-ray powder pattern for $\mathrm{Sr}_{13} \mathrm{CaCu}_{24} \mathrm{O}_{41}$

\begin{tabular}{|c|c|c|c|c|c|c|c|c|c|c|c|c|c|c|c|c|}
\hline 20 & PEAK & $d$ & $h$ & $k$ & & NTII & $\overline{(P K) !}$ & $1(D S)$ & $2 \theta$ & PEAK & $d$ & $h$ & $k l$ & I(INT) & $\overline{(\mathrm{PK})}$ & $\overline{I(D S)}$ \\
\hline 13.25 & 13.26 & 6.677 & 0 & 2 & 0 & 2 & 3 & 1 & 47.98 & & 1.8945 & 0 & 214 & 7 & & 12 \\
\hline 15.47 & 15.48 & 5.724 & 2 & 0 & 0 & 1 & 1 & 0 & 48.72 & 48.72 & 1.8675 & 2 & 014 & 3 & 3 & 6 \\
\hline 16.37 & 16.38 & 5.411 & 1 & 1 & 4 & 0 & 0 & 0 & 49.65 & 49.66 & 1.8346 & 6 & 20 & $0 \quad 12$ & 10 & 21 \\
\hline 20.42 & 20.44 & 4.346 & 2 & 2 & 0 & 0 & 0 & 0 & 50.20 & 50.38 & 1.8157 & 1 & 74 & 40 & 17 & 0 \\
\hline 22.60 & 22.62 & 3.931 & 2 & 2 & 3 & 1 & 1 & 1 & 50.38 & & 1.8099 & 5 & 37 & 720 & & 37 \\
\hline 24.73 & 24.74 & 3.597 & 1 & 1 & 7 & 8 & 8 & 6 & 50.72 & 50.72 & 1.7985 & 2 & 214 & 3 & 4 & 6 \\
\hline 25.01 & & 3.558 & 1 & 3 & 4 & 0 & & 0 & 52.01 & 52.02 & 1.7569 & 4 & 60 & 1 & 1 & 1 \\
\hline 26.68 & 26.68 & 3.339 & 0 & 4 & 0 & 17 & 17 & 14 & 52.28 & 52.28 & 1.7485 & 5 & 110 & 4 & 4 & 9 \\
\hline 30.98 & 31.00 & 2.884 & 2 & 4 & 010 & 001 & 100 & 100 & 53.34 & 53.34 & 1.7161 & 3 & 510 & 3 & 3 & 6 \\
\hline 31.23 & 31.24 & 2.862 & 4 & 0 & 0 & 10 & 87 & 10 & 53.92 & 53.92 & 1.6989 & 1 & 77 & 4 & 3 & 8 \\
\hline 31.24 & & 2.861 & 1 & 3 & 7 & 73 & & 73 & 54.96 & 54.96 & 1.6693 & 0 & 80 & $0 \quad 14$ & 12 & 29 \\
\hline 32.51 & 32.52 & 2.752 & 2 & 4 & 3 & 0 & 1 & 0 & 55.42 & 55.42 & 1.6565 & 6 & 40 & 01 & 1 & 1 \\
\hline 33.30 & 33.30 & 2.689 & 3 & 1 & 7 & 60 & 57 & 65 & 56.04 & 56.04 & 1.6397 & 5 & 310 & 2 & 2 & 5 \\
\hline 33.99 & 34.06 & 2.636 & 1 & 11 & 10 & 3 & 13 & 4 & 56.41 & 56.42 & 1.6299 & 2 & 414 & $4 \quad 15$ & 13 & 33 \\
\hline 34.05 & & 2.631 & 4 & 2 & 0 & 11 & & 12 & 56.56 & 56.56 & 1.6259 & 4 & 014 & 2 & 9 & 3 \\
\hline 36.89 & 36.90 & 2.434 & 1 & 5 & 4 & 0 & $D$ & 0 & 57.91 & 57.92 & 1.5911 & 5 & 57 & 6 & 5 & 13 \\
\hline 38.50 & 38.50 & 2.336 & 3 & 3 & 7 & 5 & 5 & 7 & 58.37 & 58.36 & 1.5797 & 4 & 214 & 2 & 2 & 4 \\
\hline 39.11 & 39.12 & 2.301 & 1 & 31 & 10 & 3 & 3 & 5 & 58.91 & 58.90 & 1.5665 & 3 & 77 & 714 & 11 & 32 \\
\hline 40.50 & 40.50 & 2.226 & 0 & 6 & 0 & 3 & 3 & 4 & 59.35 & 59.34 & 1.5558 & 1 & 710 & 1 & 2 & 3 \\
\hline 40.82 & 40.82 & 2.209 & 3 & 11 & 10 & 10 & 9 & 13 & 61.73 & 61.74 & 1.5015 & 7 & 17 & 1 & 0 & 1 \\
\hline 41.53 & 41.54 & 2.173 & 4 & 4 & 0 & 15 & 33 & 21 & 62.85 & 62.84 & 1.4775 & 0 & 614 & 5 & 4 & 13 \\
\hline 41.53 & & 2.173 & 1 & 5 & 7 & 22 & & 31 & 63.12 & 63.02 & 1.4718 & 5 & 510 & 2 & 3 & 5 \\
\hline 43.60 & 43.60 & 2.074 & 2 & 6 & 0 & 2 & 2 & 3 & 63.60 & 63.60 & 1.4617 & 4 & 414 & 411 & 9 & 27 \\
\hline 44.74 & 44.76 & 2.024 & 2 & 6 & 3 & 0 & 1 & 1 & 64.07 & 64.06 & 1.4522 & 3 & 710 & 1 & 1 & 3 \\
\hline 45.29 & 45.30 & 2.001 & 3 & 31 & 10 & 7 & 6 & 11 & & & & & & & & \\
\hline 45.90 & 45.90 & 1.9756 & 0 & 01 & 14 & 18 & 16 & 29 & & & & & & & & \\
\hline 46.29 & 46.28 & 1.9596 & 5 & 1 & 7 & 1 & 2 & 2 & & & & & & & & \\
\hline 46.46 & & 1.9531 & 5 & 3 & 4 & 0 & & 0 & & & & & & & & \\
\hline 47.62 & 47.62 & 1.9080 & 6 & 0 & 0 & 10 & 9 & 18 & & & & & & & & \\
\hline 47.97 & 47.98 & 1.8948 & 1 & 51 & 10 & 1 & 7 & 1 & & & & & & & & \\
\hline
\end{tabular}


Table C.3. Simulated X-ray powder pattern for $\mathrm{Sr}_{12} \mathrm{Ca}_{2} \mathrm{Cu}_{24} \mathrm{O}_{41}$

\begin{tabular}{|c|c|c|c|c|c|c|c|c|c|c|c|c|c|c|c|c|c|}
\hline $2 \theta$ & PEAK & d & $h$ & $k$ & $\overline{l l(1)}$ & $\overline{N T I !}$ & $|(\mathrm{PK})|$ & (DS) & $2 \theta$ & PEAK & $\bar{d}$ & $h$ & k & & INT) & $\overline{(\mathrm{PK})}$ & (DS) \\
\hline 13.29 & 13.30 & 6.657 & 0 & 2 & 0 & 3 & 3 & 1 & 48.00 & 48.00 & 1.8940 & 0 & & 14 & 7 & 7 & 13 \\
\hline 15.48 & 15.50 & 5.719 & 2 & 0 & 0 & 0 & 0 & 0 & 48.06 & & 1.8918 & 1 & & 10 & 1 & & 1 \\
\hline 16.38 & 16.40 & 5.407 & 1 & 1 & 4 & 0 & 0 & 0 & 48.72 & 48.72 & 1.8674 & 2 & & 14 & 3 & 3 & 5 \\
\hline 20.46 & 20.46 & 4.338 & 2 & 2 & 0 & 0 & 0 & 0 & 49.71 & 49.72 & 1.8327 & 6 & 2 & 0 & 12 & 10 & 22 \\
\hline 22.64 & 22.64 & 0.925 & 2 & 2 & 3 & 1 & 1 & 1 & 50.36 & 50.44 & 1.8106 & 1 & 7 & 4 & 0 & 17 & 0 \\
\hline 24.74 & 24.74 & 3.596 & 1 & 1 & 7 & 7 & 7 & 6 & 50.43 & & 1.8080 & 5 & 3 & 7 & 19 & & 36 \\
\hline 25.06 & & 3.550 & 1 & 3 & 4 & 0 & & 0 & 50.74 & 50.72 & 1.7980 & 2 & & 14 & 3 & 5 & 6 \\
\hline 26.76 & 26.78 & 3.328 & 0 & 4 & 0 & 19 & 19 & 16 & 52.13 & 52.30 & 1.7530 & 4 & 6 & 0 & 1 & 4 & 1 \\
\hline 31.06 & 31.08 & 2.877 & 2 & 4 & 01 & 00 & 100 & 100 & 52.31 & & 1.7475 & 5 & & 10 & 5 & & 9 \\
\hline 31.26 & 31.28 & 2.859 & 4 & 0 & 0 & 9 & 88 & 9 & 53.43 & 53.42 & 1.7136 & 3 & & 10 & 3 & 3 & 6 \\
\hline 31.28 & & 2.857 & 1 & 3 & 7 & 72 & & 72 & .07 & 54.06 & 1.6948 & 1 & 7 & 7 & 3 & 3 & 7 \\
\hline 32.58 & 32.60 & 2.746 & 2 & 4 & 3 & 0 & 1 & 0 & 15 & 55.14 & 1.6641 & 0 & 8 & 0 & 14 & 11 & 29 \\
\hline 33.32 & 33.32 & 2.687 & 3 & 1 & 7 & 60 & 56 & 65 & .51 & 55.28 & 1.6542 & 6 & 4 & 0 & 1 & 7 & 1 \\
\hline 33.99 & 34.10 & 2.635 & 1 & 1 & 10 & 3 & 13 & 4 & 56.09 & 56.10 & 1.6383 & 5 & & 10 & 2 & 2 & 5 \\
\hline 34.10 & & 2.627 & 4 & 2 & 0 & 11 & & 12 & 56.46 & 56.46 & 1.6285 & 2 & & 14 & 15 & 13 & 32 \\
\hline 36.99 & 37.00 & 2.428 & 1 & 5 & 4 & 0 & 0 & 0 & 8 & 0 & 1.6254 & 4 & & 14 & 1 & 9 & 3 \\
\hline 38.55 & 38.56 & 2.334 & 3 & 3 & 7 & 5 & 4 & 6 & 8.01 & 58.00 & 1.5887 & 5 & 5 & 7 & 6 & 5 & 13 \\
\hline 39.15 & 39.14 & 2.299 & 1 & 3 & 10 & 4 & 3 & 5 & 58.40 & 58.40 & 1.5790 & 4 & & 14 & 2 & 2 & 4 \\
\hline 40.63 & 40.64 & 2.219 & 0 & 6 & 0 & 3 & 4 & 4 & 59.05 & 59.06 & 1.5631 & 3 & 7 & 7 & 14 & 11 & 31 \\
\hline 40.84 & 40.84 & 2.208 & 3 & & 10 & 10 & 10 & 14 & 59.49 & 59.48 & 1.5526 & 1 & & 10 & 1 & 2 & 3 \\
\hline 41.61 & 41.62 & 2.169 & 4 & 4 & 0 & 15 & 32 & 21 & 61.78 & 61.78 & 1.5004 & 7 & 1 & 7 & 0 & 0 & 1 \\
\hline 41.62 & & 2.168 & 1 & 5 & 7 & 22 & & 31 & 62.94 & 62.94 & 1.4755 & 0 & & 14 & 5 & 4 & 13 \\
\hline 43.72 & 43.72 & 2.069 & 2 & 6 & 0 & 2 & 2 & 3 & 63.21 & 63.12 & 1.4699 & 5 & & 10 & 2 & 3 & 5 \\
\hline 44.87 & 44.88 & 2.018 & 2 & 6 & 3 & 0 & 1 & 1 & 63.66 & 63.66 & 1.4606 & 4 & & 14 & 10 & 8 & 27 \\
\hline 45.33 & 45.34 & 1.9988 & 3 & 3 & 10 & 7 & 6 & 11 & & & & & & & & & \\
\hline 45.90 & 45.90 & 1.9756 & 0 & 0 & 14 & 18 & 16 & 29 & & & & & & & & & \\
\hline 46.33 & 46.32 & 1.9582 & 5 & 1 & 7 & 1 & 2 & 2 & & & & & & & & & \\
\hline 46.52 & & 1.9507 & 5 & 3 & 4 & 0 & & ( & & & & & & & & & \\
\hline 47.55 & 47.66 & 1.9108 & 3 & 5 & 7 & 0 & 9 & 0 & & & & & & & & & \\
\hline 47.67 & & 1.9063 & 6 & 0 & 0 & 10 & & 18 & & & & & & & & & \\
\hline
\end{tabular}


Table C.4. Simulated X-ray powder pattern for $\mathrm{Sr}_{11} \mathrm{Ca}_{3} \mathrm{Cu}_{24} \mathrm{O}_{41}$

\begin{tabular}{|c|c|c|c|c|c|c|c|c|c|c|c|c|c|c|c|c|c|}
\hline \multirow{2}{*}{$\frac{2 \theta}{13.35}$} & \multirow{2}{*}{$\begin{array}{c}\text { PEAK } \\
13.36\end{array}$} & \multirow{2}{*}{$\begin{array}{c}\frac{d}{6.628} \\
6.62\end{array}$} & \multirow{2}{*}{ h } & \multirow{2}{*}{$\frac{k}{2}$} & \multicolumn{4}{|c|}{$\ell|(\mid N T)|(P K) \mid(D S)$} & \multirow{2}{*}{$\frac{2 \theta}{48.10}$} & \multirow{2}{*}{$\begin{array}{c}\text { PEAK } \\
48.10\end{array}$} & \multirow{2}{*}{$\frac{d}{1.8903}$} & \multirow{2}{*}{$\frac{h}{0}$} & \multirow{2}{*}{ 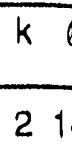 } & \multicolumn{4}{|c|}{$\ell|(\mid N T)|(P K) \mid(D S)$} \\
\hline & & & & & 0 & 3 & 3 & 1 & & & & & & 4 & 8 & 7 & 13 \\
\hline 15.51 & 15.52 & 5.710 & 2 & 0 & 0 & 0 & 0 & 0 & 48.21 & 48.22 & 1.8861 & 1 & 510 & & 1 & 5 & 1 \\
\hline 16.41 & 16.42 & 5.396 & 1 & 1 & 4 & 0 & 0 & 0 & 48.82 & 48.82 & 1.8641 & 2 & 016 & 4 & 3 & 2 & 5 \\
\hline 20.51 & 20.52 & 4.326 & 2 & 2 & 0 & 0 & 0 & 0 & 49.80 & 49.80 & 1.8294 & 6 & 2 & 0 & 13 & 11 & 23 \\
\hline 22.69 & 22.70 & 3.915 & 2 & 2 & 3 & 1 & 1 & 1 & 50.55 & 50.54 & 1.8043 & 5 & 3 & 7 & 19 & 16 & 36 \\
\hline 24.79 & 24.80 & 3.589 & 1 & 1 & 7 & 6 & 6 & 5 & 50.57 & & 1.8034 & 1 & 7 & 4 & 0 & & 0 \\
\hline 25.15 & 25.14 & 3.538 & 1 & 3 & 4 & 0 & 1 & 0 & 50.84 & 50.82 & 1.7945 & 2 & 21 & 4 & 4. & 5 & 7 \\
\hline 26.88 & 26.88 & 3.314 & 0 & 4 & 0 & 22 & 21 & 18 & 52.31 & 52.40 & 1.7473 & 4 & 6 & 0 & 1 & 4 & 2 \\
\hline 31.18 & 31.18 & 2.866 & 2 & 4 & 01 & 1001 & 1001 & 100 & 52.40 & & 1.7446 & 5 & 11 & 0 & 5 & & 9 \\
\hline 31.31 & & 2.855 & 4 & 0 & 0 & 9 & & 9 & 53.59 & 53.58 & 1.7089 & 3 & 51 & 0 & 3 & 3 & 7 \\
\hline 31.37 & 31.36 & 2.850 & 1 & 3 & 7 & 71 & 87 & 71 & 54.29 & 54.28 & 1.6885 & 1 & 7 & 7 & 3 & 3 & 6 \\
\hline 32.70 & 32.72 & 2.737 & 2 & 4 & 3 & 0 & 1 & 0 & 55.40 & 55.40 & 1.6571 & 0 & 8 & 0 & 14 & 11 & 29 \\
\hline 33.38 & 33.38 & 2.682 & 3 & 1 & 7 & 60 & 54 & 64 & 55.64 & 55.54 & 1.6505 & 6 & 4 & 0 & 0 & 7 & 1 \\
\hline 34.06 & 34.16 & 2.630 & 1 & 11 & 10 & 3 & 12 & 4 & 56.22 & 56.22 & 1.6350 & 5 & 31 & 10 & 2 & 2 & 5 \\
\hline 34.17 & & 2.622 & 4 & 2 & 0 & 11 & & 13 & 56.60 & 56.60 & 1.6247 & 2 & 41 & 4 & 15 & 12 & 32 \\
\hline 37.14 & 37.14 & 2.419 & 1 & 5 & 4 & 0 & 0 & 0 & 56.68 & & 1.6226 & 4 & 01 & 14 & 1 & & 3 \\
\hline 38.64 & 38.64 & 2.328 & 3 & 3 & 7 & 4 & 4 & 5 & 58.17 & 58.18 & 1.5847 & 5 & 5 & 7 & 6 & 4 & 12 \\
\hline 39.24 & 39.24 & 2.294 & 1 & 31 & 10 & 4 & 3 & 5 & 58.51 & 58.52 & 1.5761 & 4 & 21 & 14 & 2 & 2 & 4 \\
\hline 40.81 & 40.92 & 2.209 & 0 & 6 & 0 & 3 & 11 & 4 & 59.27 & 59.28 & 1.5578 & 3 & 7 & 7 & 13 & 11 & 31 \\
\hline 40.92 & & 2.204 & 3 & 11 & 10 & 11 & & 15 & 59.71 & 59.70 & 1.5474 & 1 & 71 & 10 & 1 & 2 & 3 \\
\hline 41.72 & 41.76 & 2.163 & 4 & 4 & 0 & 14 & 28 & 20 & 61.89 & 61.90 & 1.4979 & 7 & 1 & 7 & 0 & 0 & 1 \\
\hline 41.77 & & 2.161 & 1 & 5 & 7 & 22 & & 31 & 63.14 & 63.14 & 1.4713 & 0 & 61 & 14 & 5 & 4 & 14 \\
\hline 43.90 & 43.90 & 2.061 & 2 & 6 & 0 & 2 & 2 & 3 & 63.38 & 63.32 & 1.4663 & 5 & 51 & 10 & 2 & 3 & 5 \\
\hline 45.05 & 45.06 & 2.011 & 2 & 6 & 3 & 0 & 1 & 1 & 63.82 & 63.82 & 1.4574 & 4 & 41 & 14 & 10 & 8 & 26 \\
\hline 45.44 & 45.44 & 1.9944 & 3 & 31 & 10 & 7 & 6 & 11 & & & & & & & & & \\
\hline 45.98 & 45.98 & 1.9721 & 0 & 01 & 14 & 18 & 15 & 29 & & & & & & & & & \\
\hline 46.41 & 46.40 & 1.9549 & 5 & 1 & 7 & 1 & 2 & 2 & & & & & & & & & \\
\hline 46.62 & & 1.9466 & 5 & 3 & 4 & 0 & & 0 & & & & & & & & & \\
\hline 47.70 & 47.74 & 1.9051 & 3 & 5 & 7 & 0 & 9 & 0 & & & & & & & & & \\
\hline 47.75 & & 1.9033 & 6 & 0 & 0 & 10 & & 17 & & & & & & & & & \\
\hline
\end{tabular}


Table C.5. Simulated X-ray powder pattern for $\mathrm{Sr}_{10} \mathrm{Ca}_{4} \mathrm{Cu}_{24} \mathrm{O}_{41}$

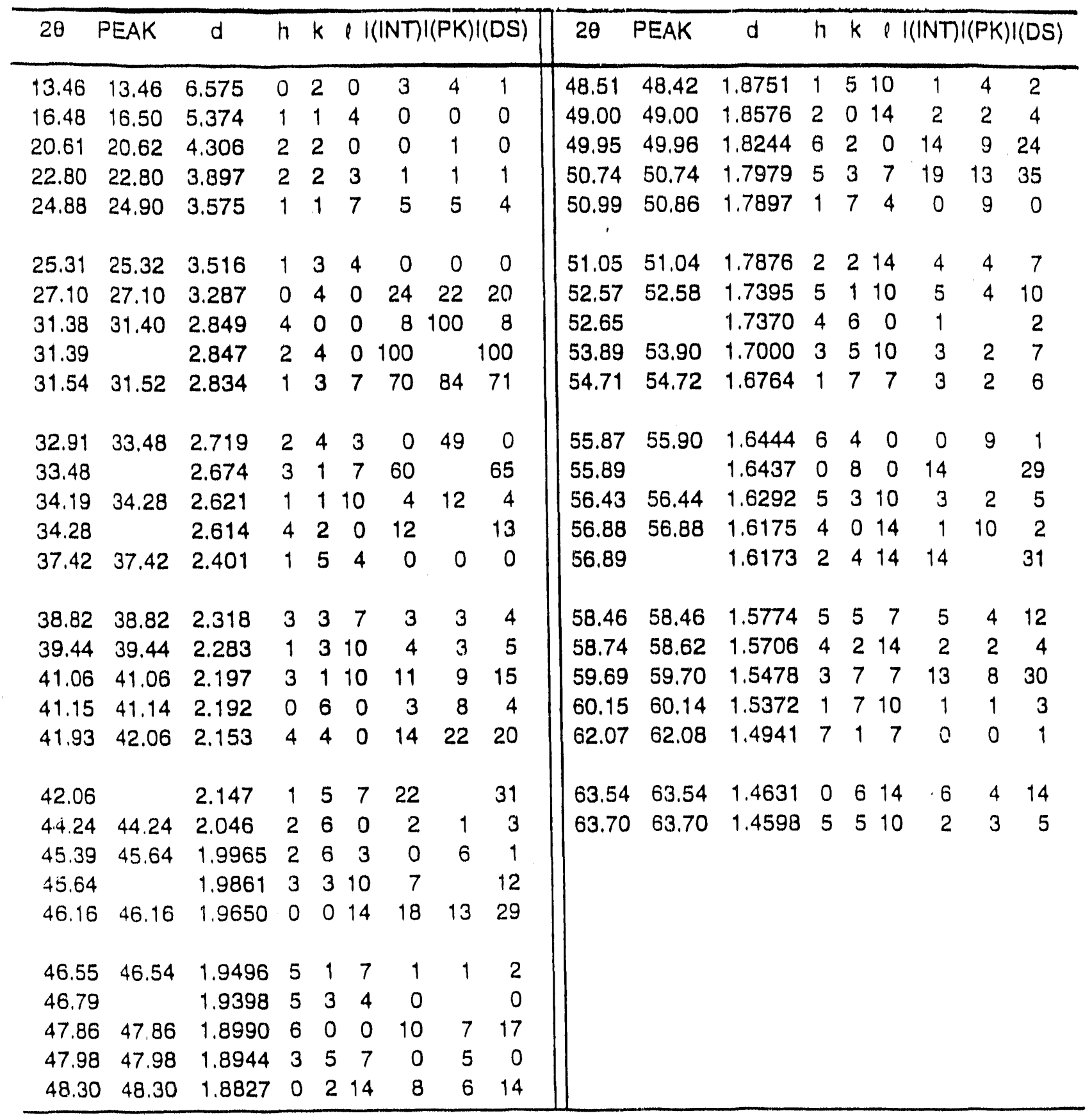


Table C.6. Simulated $\mathrm{X}$-ray powder pattern for $\mathrm{Sr}_{9} \mathrm{Ca}_{5} \mathrm{Cu}_{24} \mathrm{O}_{41}$

\begin{tabular}{|c|c|c|c|c|c|c|c|c|c|c|c|c|c|c|c|c|c|}
\hline \multirow{2}{*}{20} & \multirow{2}{*}{$\frac{\text { PEAK }}{13.56}$} & \multirow{2}{*}{$\frac{d}{6.530}$} & \multirow{2}{*}{$\frac{h}{0}$} & \multirow{2}{*}{$\frac{k}{2}$} & \multicolumn{4}{|c|}{$\ell|(\mid N T)|(P K) \mid(D S)$} & \multirow{2}{*}{$\frac{2 \theta}{48.43}$} & \multirow{2}{*}{$\frac{\text { PEAK }}{48.44}$} & \multirow{2}{*}{$\frac{d}{1.8779}$} & \multirow{2}{*}{$\frac{h}{0}$} & \multirow{2}{*}{$\frac{k}{21}$} & \multicolumn{4}{|c|}{ l $|(\mid N T)|(P K) \mid(D S)$} \\
\hline & & & & & 0 & 3 & 3 & 2 & & & & & & 14 & 9 & 6 & 15 \\
\hline 15.56 & 15.58 & 5.690 & 2 & 0 & 0 & 0 & 0 & 0 & 48.75 & 48.72 & 1.8666 & 1 & 5 & 10 & 1 & 1 & 2 \\
\hline 16.53 & 16.54 & 5.359 & 1 & 1 & 4 & 0 & 0 & 0 & 49.11 & 49.10 & 1.8537 & 2 & 0 & 14 & 2 & 2 & 4 \\
\hline 20.69 & 20.70 & 4.290 & 2 & 2 & 0 & 1 & 0 & 0 & 50.04 & 50.04 & 1.8214 & 6 & 2 & 0 & 14 & 10 & 26 \\
\hline 22.88 & 22.88 & 3.884 & 2 & 2 & 3 & 1 & 1 & 1 & 50.87 & 50.86 & 1.7936 & 5 & 3 & 7 & 19 & 13 & 35 \\
\hline 24.95 & 24.96 & 3.567 & 1 & 1 & 7 & 4 & 3 & 3 & 51.18 & 51.18 & 1.7833 & 2 & 2 & 14 & 4 & 4 & 7 \\
\hline 25.44 & 25.44 & 3.498 & 1 & 3 & 4 & 0 & 0 & 0 & 51.34 & 51.30 & 1.7782 & 1 & 7 & 4 & 0 & 2 & 0 \\
\hline 27.29 & 27.30 & 3.265 & 0 & 4 & 0 & 27 & 21 & 23 & 52.67 & 52.66 & 1.7365 & 5 & 1 & 10 & 5 & 4 & 10 \\
\hline 31.42 & 31.58 & 2.845 & 4 & 0 & 0 & 8 & 93 & 8 & 52.92 & 52.80 & 1.7287 & 4 & 6 & 0 & 1 & 2 & 2 \\
\hline 31.57 & & 2.832 & 2 & 4 & 0 & 100 & & 100 & 54.12 & 54.12 & 1.6933 & 3 & 5 & 10 & 4 & 2 & 7 \\
\hline 31.67 & 31.66 & 2.823 & 1 & 3 & 7 & 69 & 100 & 70 & 54.55 & 54.54 & 1.6809 & 0 & 4 & 14 & 0 & 0 & 0 \\
\hline 33.09 & 33.56 & 2.705 & 2 & 4 & 3 & 0 & 46 & 0 & 55.06 & 55.06 & 1.6666 & 1 & 7 & 7 & 3 & 2 & 5 \\
\hline 33.55 & & 2.669 & 3 & 1 & 7 & 60 & & 65 & 56.03 & 56.32 & 1.6400 & 6 & 4 & 0 & 0 & 9 & 1 \\
\hline 34.27 & 34.36 & 2.614 & 1 & 1 & 10 & 4 & 12 & 4 & 56.31 & & 1.6325 & 0 & 8 & 0 & 14 & & 29 \\
\hline 34.36 & & 2.608 & 4 & 2 & 0 & 12 & & 14 & 56.58 & 56.46 & 1.6254 & 5 & 3 & 10 & 3 & 6 & 6 \\
\hline 37.66 & 37.66 & 2.387 & 1 & 5 & 4 & 0 & 0 & 0 & 57.00 & 57.10 & 1.6144 & 4 & 0 & 14 & 1 & 10 & 2 \\
\hline 38.95 & 38.96 & 2.311 & 3 & 3 & 7 & 3 & 2 & 4 & 57.09 & & 1.6120 & 2 & 4 & 14 & 14 & & 30 \\
\hline 39.58 & 39.58 & 2.275 & 1 & 3 & 10 & 4 & 3 & 5 & 58.68 & 58.68 & 1.5720 & 5 & 5 & 7 & 5 & 4 & 12 \\
\hline 41.14 & 41.14 & 2.192 & 3 & 1 & 10 & 12 & 9 & 16 & 58.88 & 58.86 & 1.5673 & 4 & 2 & 14 & 2 & 3 & 4 \\
\hline 41.45 & 41.46 & 2.177 & 0 & $\theta$ & 0 & 3 & 3 & 4 & 60.04 & 60.04 & 1.5397 & 3 & 7 & 7 & 13 & 9 & 29 \\
\hline 42.09 & 42.10 & 2.145 & 4 & 4 & 0 & 13 & 11 & 19 & 60.50 & 60.50 & 1.5290 & 1 & 7 & 10 & 1 & 1 & 3 \\
\hline 42.29 & 42.30 & 2.135 & 1 & 5 & 7 & 22 & 17 & 31 & 62.17 & 62.18 & 1.4919 & 7 & 1 & 7 & 0 & 0 & 1 \\
\hline 44.53 & 44.54 & 2.033 & 2 & 6 & 0 & 2 & 1 & 2 & 63.84 & 63.84 & 1.4568 & 0 & 6 & 14 & 6 & 4 & 14 \\
\hline 45.68 & 45.78 & 1.9846 & 2 & 6 & 3 & 0 & 6 & 1 & 63.93 & & 1.4550 & 5 & 5 & 10 & 2 & & 5 \\
\hline 45.78 & & 1.9804 & 3 & 3 & 10 & 8 & & 12 & & & & & & & & & \\
\hline 46.27 & 46.26 & 1.9607 & 0 & 0 & 14 & 18 & 13 & 29 & & & & & & & & & \\
\hline 46.62 & 46.38 & 1.9465 & 5 & 1 & 7 & 1 & 8 & 1 & & & & & & & & & \\
\hline 46.91 & & 1.9351 & 5 & 3 & 4 & 0 & & 0 & & & & & & & & & \\
\hline 47.92 & 47.92 & 1.8967 & 6 & 0 & 0 & 10 & 7 & 17 & & & & & & & & & \\
\hline 48.21 & 48.04 & 1.8861 & 3 & 5 & 7 & 0 & 5 & 1 & & & & & & & & & \\
\hline
\end{tabular}


Table C.7. Simulated X-ray powder pattern for $\mathrm{Sr}_{8} \mathrm{Cu}_{6} \mathrm{Cu}_{24} \mathrm{O}_{41}$

\begin{tabular}{|c|c|c|c|c|c|c|c|c|c|c|c|c|c|c|c|}
\hline \multirow{2}{*}{$\frac{2 \theta}{13.60}$} & \multirow{2}{*}{$\frac{\text { PEAK }}{13.62}$} & \multirow{2}{*}{$\frac{d}{6.505}$} & \multirow{2}{*}{$\frac{h}{0}$} & \multirow{2}{*}{$\frac{k}{2}$} & \multicolumn{3}{|c|}{ l I(INT)I(PK)I(DS) } & \multirow{2}{*}{$\frac{20}{48.47}$} & \multirow{2}{*}{ PEAK } & \multirow{2}{*}{$\frac{d}{1.8767}$} & \multirow{2}{*}{$\frac{5}{0}$} & \multicolumn{4}{|c|}{$k \quad l|(I N T)|(P K) \mid(D S)$} \\
\hline & & & & & 4 & 3 & 2 & & & & & 214 & 9 & & 15 \\
\hline 15.57 & 15.58 & 5.688 & 2 & 00 & 1 & 1 & 0 & 48.86 & 48.86 & $1.862 \theta$ & 1 & 510 & 1 & 1 & 2 \\
\hline 16.54 & 16.56 & 5.354 & 1 & 14 & 0 & 0 & 0 & 49.13 & 49.12 & 1.8531 & 2 & 014 & 2 & 1 & 3 \\
\hline 20.73 & 20.74 & 4.282 & 2 & 20 & 1 & 0 & 0 & 50.08 & 50.08 & 1.8201 & 6 & 20 & 15 & 10 & 27 \\
\hline 22.91 & 22.92 & 3.878 & 2 & 23 & 1 & 1 & 1 & 50.92 & 50.92 & 1.7918 & 5 & 37 & 19 & 12 & 34 \\
\hline 24.96 & 24.98 & 3.564 & 11 & 17 & 3 & 2 & 3 & 51.22 & 51.20 & 1.7822 & 2 & 214 & 4 & 4 & 7 \\
\hline 25.51 & 25.52 & 3.489 & 13 & 34 & 0 & 0 & 0 & 51.53 & 51.34 & 1.7720 & 1 & 74 & 0 & 2 & 0 \\
\hline 27.40 & 27.40 & 3.253 & 06 & 40 & $0 \quad 30$ & 21 & 25 & 52.69 & 52.70 & 1.7357 & 5 & 110 & 6 & 3 & 11 \\
\hline 31.43 & 31.72 & 2.844 & 4 & 00 & 071 & 100 & 7 & 53.07 & 53.06 & 1.7243 & 4 & 60 & 1 & 1 & 2 \\
\hline 31.66 & & 2.823 & 2 & 40 & 0100 & & 100 & 54.23 & 54.22 & 1.6901 & 3 & 510 & 4 & 2 & 7 \\
\hline 31.73 & & 2.817 & 13 & 37 & 768 & & 69 & 54.63 & 54.62 & 1.6787 & 0 & 414 & 0 & 0 & 0 \\
\hline 33.18 & 33.58 & 2.698 & 2 & 43 & 0 & 41 & 0 & 55.25 & 55.24 & 1.6614 & 1 & 77 & 2 & 1 & 5 \\
\hline 33.57 & & 2.667 & 3 & 17 & 760 & & 64 & 56.11 & 56.54 & 1.6379 & 6 & 40 & 0 & 9 & 0 \\
\hline 34.29 & 34.40 & 2.613 & 1 & 110 & 4 & 11 & 4 & 56.55 & & 1.6263 & 0 & 80 & 14 & & 29 \\
\hline 34.39 & & 2.606 & 4 & 20 & 013 & & 14 & 56.63 & & 1.6239 & 5 & 310 & 3 & & 6 \\
\hline 37.78 & 37.78 & 2.379 & 1 & 54 & 0 & 0 & 0 & 57.02 & 57.16 & 1.6138 & 4 & 014 & 1 & 9 & 2 \\
\hline 39.00 & 39.00 & 2,308 & 3 & 37 & 2 & 2 & 3 & 57.17 & & 1.6101 & 2 & 414 & 14 & & 29 \\
\hline 39.64 & 39.64 & 2.272 & 1 & 310 & 4 & 3 & 6 & 58.79 & 58.80 & 1.5694 & 5 & 57 & 5 & 3 & 12 \\
\hline 41.16 & 41.16 & 2.191 & 3 & 110 & $0 \quad 12$ & 8 & 17 & 58.92 & 58.94 & 1.5663 & 4 & 214 & 2 & 3 & 4 \\
\hline 41.62 & 41.62 & 2.168 & 0 & 60 & 3 & 2 & 4 & 60.22 & 60.22 & 1.5355 & 3 & 77 & 13 & 8 & 29 \\
\hline 42.18 & 42.18 & 2.141 & 4 & 40 & $0 \quad 13$ & 9 & 18 & 60.68 & 60.68 & 1.5249 & 1 & 710 & 1 & 1 & 3 \\
\hline 42.41 & 42.42 & 2.130 & 1 & 57 & $\begin{array}{ll}7 \quad 22 \\
\end{array}$ & 15 & 31 & 62.20 & 62.20 & 1.4912 & 7 & 17 & 0 & 0 & 0 \\
\hline 44.69 & 44.70 & 2.026 & 2 & 60 & 2 & 1 & 2 & 63.98 & 64.00 & 1.4540 & 0 & 614 & 6 & 4 & 14 \\
\hline 45.83 & 45.84 & 1.9782 & 3 & 310 & 8 & 6 & 13 & 64.04 & & 1.4529 & 5 & 510 & 2 & & 5 \\
\hline 45.84 & & 1.9781 & 2 & 63 & 0 & & 1 & & & & & & & & \\
\hline 46.28 & 46.28 & 1.9600 & 0 & 016 & $14 \quad 18$ & 12 & 29 & & & & & & & & \\
\hline 46.65 & 46.40 & 1.9455 & 5 & 17 & 1 & 8 & 1 & & & & & & & & \\
\hline 46.97 & & 1.9330 & 5 & 3 & 0 & & 0 & & & & & & & & \\
\hline 47.95 & 47.94 & 1.8958 & 6 & 0 & $0 \quad 10$ & 6 & 17 & & & & & & & & \\
\hline 48.32 & 48.46 & 1.8820 & 3 & 57 & 1 & 6 & 1 & & & & & & & & \\
\hline
\end{tabular}


Table C.8. Simulated $\mathrm{X}$-ray powder paltern for $\mathrm{Sr}_{7} \mathrm{Ca}_{7} \mathrm{Cu}_{24} \mathrm{O}_{41}$

\begin{tabular}{|c|c|c|c|c|c|c|c|c|c|c|c|c|c|c|c|c|}
\hline $2 \theta$ & $\overline{P E A K}$ & $\bar{d}$ & $h$ & $k$ & $\overline{l \mid 11}$ & $\overline{N T T I}$ & $\overline{(\mathrm{PK}) \mid}$ & (DS) & 20 & PEAK & $d$ & $h$ & $k 1 T$ & $\overline{\mid N T) \mid}$ & $\overline{(P K) !}$ & $\overline{(D S)}$ \\
\hline 13.63 & 13.64 & 6.493 & 0 & 2 & 0 & 4 & 3 & 2 & 48.56 & & 1.8734 & 0 & 214 & 10 & & 16 \\
\hline 15.57 & 15.58 & 5.686 & 2 & 0 & 0 & 2 & 1 & 1 & 48.95 & 48.94 & 1.8593 & 1 & 510 & 1 & 1 & 2 \\
\hline 16.57 & 16.58 & 5.346 & 1 & 1 & 4 & 0 & 0 & 0 & 49.21 & 49.20 & 1.8501 & 2 & 014 & 2 & 1 & 3 \\
\hline 20.75 & 20.76 & 4.277 & 2 & 2 & 0 & 1 & 1 & 0 & 50.10 & 50.10 & 1.8193 & 6 & 20 & 16 & 10 & 28 \\
\hline 22.94 & 22.96 & 3.873 & 2 & 2 & 3 & 1 & 1 & 1 & 50.97 & 50.98 & 1.7902 & 5 & 37 & 19 & 12 & 33 \\
\hline 25.00 & 25.02 & 3.559 & 1 & 1 & 7 & 2 & 2 & 2 & 51.31 & 51.30 & 1.7793 & 2 & 214 & 4 & 3 & 7 \\
\hline 25.55 & 25.56 & 3.483 & 1 & 3 & 4 & 0 & 0 & 0 & 51.63 & 51.42 & 1.7688 & 1 & 74 & 0 & 2 & 0 \\
\hline 27.45 & 27.46 & 3.247 & 0 & 4 & 0 & 33 & 24 & 28 & 52.75 & 52.74 & 1.7341 & 5 & 110 & $\theta$ & 4 & 11 \\
\hline 31.44 & 31.78 & 2.843 & 4 & 0 & 0 & 7 & 100 & 7 & 53.14 & 53.14 & 1.7220 & 4 & 60 & $i$ & 1 & 2 \\
\hline 31.71 & & 2.819 & 2 & 4 & 01 & 00 & & 100 & 54.32 & 54.32 & 1.6876 & 3 & 510 & 4 & 2 & 8 \\
\hline 31.79 & & 2.813 & 1 & 3 & 7 & 67 & & 67 & 54.73 & 54.72 & 1.6758 & 0 & 414 & 0 & 0 & 1 \\
\hline 33.23 & 33.62 & 2.694 & 2 & 4 & 3 & 0 & 41 & 0 & 55.35 & 55.36 & 1.6584 & 1 & 77 & 2 & 1 & 4 \\
\hline 33.61 & & 2.665 & 3 & 1 & 7 & 60 & & 64 & 56.15 & 56.66 & 1.6367 & 6 & 40 & 0 & 10 & 0 \\
\hline 34.35 & 34,42 & 2.609 & 1 & 1 & 10 & 4 & 12 & 5 & 56.66 & & 1.6233 & 0 & 80 & 14 & & 29 \\
\hline 34.41 & & 2.604 & 4 & 2 & 0 & 13 & & 15 & 56.70 & & 1.6222 & 5 & 310 & 3 & & 6 \\
\hline 37.85 & 37.86 & 2.375 & 1 & 5 & 4 & 0 & 0 & 0 & 57.10 & 57.26 & 1.6117 & 4 & 014 & 1 & 9 & 2 \\
\hline 39.05 & 39.06 & 2.305 & 3 & 3 & 7 & 2 & 1 & 3 & 57.27 & & 1.6074 & 2 & 414 & 13 & & 28 \\
\hline 39.71 & 39.72 & 2.268 & 1 & 3 & 10 & 4 & 3 & 6 & 58.86 & 58.86 & 1.5677 & 5 & 57 & 5 & 4 & 12 \\
\hline 41.22 & 41.22 & 2.188 & 3 & 1 & 10 & 13 & 9 & 17 & 59.00 & 59.02 & 1.5642 & 4 & 214 & 2 & 3 & 4 \\
\hline 41.70 & 41.70 & 2.164 & 0 & 6 & 0 & 3 & 3 & 4 & 60.33 & 60.32 & 1.5331 & 3 & 77 & 12 & 7 & 28 \\
\hline 42.22 & 42.22 & 2.139 & 4 & 4 & 0 & 12 & 9 & 17 & 60.80 & 60.80 & 1.5222 & 1 & 710 & 2 & 1 & 4 \\
\hline 42.49 & 42.50 & 2.126 & 1 & 5 & 7 & 22 & 15 & 31 & 62.24 & 62.24 & 1.4904 & 7 & 17 & 0 & 0 & 0 \\
\hline 44.77 & 44.78 & 2.023 & 2 & 6 & 0 & 1 & 1 & 2 & & & & & & & & \\
\hline 45.90 & 45.90 & 1.9755 & 3 & 3 & 10 & 8 & 6 & 13 & & & & & & & & \\
\hline 45.92 & & 1.9749 & 2 & 6 & 3 & 1 & & 1 & & & & & & & & \\
\hline 46.37 & 46.38 & 1.9566 & 0 & 0 & 14 & 18 & 12 & 29 & & & & & & & & \\
\hline 46.68 & 46.48 & 1.9441 & 5 & 1 & 7 & 0 & 8 & 1 & & & & & & & & \\
\hline 47.01 & & 1.9315 & 5 & 3 & 4 & 0 & 0 & & & & & & & & & \\
\hline 47.96 & 47.96 & 1.8952 & 6 & 0 & 0 & 10 & 7 & 17 & & & & & & & & \\
\hline 48.40 & 48.56 & 1.8793 & 3 & 5 & 7 & 1 & 6 & 1 & & & & & & & & \\
\hline
\end{tabular}


APPENDIX D: $(\mathrm{Ca}, \mathrm{Sr}) \mathrm{CuO}_{2}$ 
Table D.1. Simulated X-ray powder pattern for $\mathrm{SrCuO}_{2}$

\begin{tabular}{|c|c|c|c|c|c|c|c|c|c|c|c|c|c|c|c|c|}
\hline $2 \theta$ & PEAK & $d$ & $\bar{h}$ & $k$ & 811 & !NT)! & $(\mathrm{PK}) \mathrm{I}$ & (DS) & $2 \theta$ & SEAK & $\bar{d}$ & $h k$ & & (NT) & $\overline{(\mathrm{PK})}$ & I(DS) \\
\hline 10.83 & 10.84 & 8.166 & 0 & 2 & 0 & 0 & 1 & 0 & 68.94 & 68.94 & 1.3609 & 012 & 0 & 2 & 2 & 5 \\
\hline 21.75 & 21.76 & 4.083 & 0 & 4 & 0 & 16 & 18 & 9 & 69.92 & 69.92 & 1.3444 & 28 & 0 & 6 & 5 & 16 \\
\hline 25.21 & 25.22 & 3.529 & 0 & 2 & 1 & 8 & 9 & 5 & 71.44 & 71.44 & 1.3194 & 20 & 2 & 10 & 9 & 29 \\
\hline 25.50 & 25.50 & 3.490 & 1 & 1 & 0 & 15 & 17 & 10 & 73.07 & 73.08 & 1.2939 & 111 & 1 & 12 & 10 & 35 \\
\hline 29.89 & 29.90 & 2.987 & 1 & 3 & 0 & 63 & 65 & 53 & 73.45 & 73.28 & 1.2882 & 02 & 3 & 0 & 6 & 1 \\
\hline 31.64 & 31.64 & 2.825 & 0 & 4 & 1 & 56 & 56 & 50 & 73.63 & 73.64 & 1.2854 & 012 & 1 & 0 & 1 & 1 \\
\hline 32.88 & 32.88 & 2.722 & 0 & 6 & 0 & 6 & 6 & 5 & 74.58 & 74.58 & 1.2715 & 28 & 1 & 4 & 4 & 13 \\
\hline 34.40 & 34.40 & 2.605 & 1 & 1 & 11 & 00 & 100 & 100 & 75.70 & 75.80 & 1.2554 & 24 & 2 & 1 & 3 & 4 \\
\hline 37.27 & 37.28 & 2.411 & 1 & 5 & 0 & 11 & 11 & 13 & 75.81 & & 1.2538 & 010 & 2 & 3 & & 8 \\
\hline 37.86 & 37.86 & 2.374 & 1 & 3 & 1 & 11 & 11 & 13 & 76.31 & 76.32 & 1.2469 & 19 & 2 & 0 & 1 & 1 \\
\hline 40.33 & 40.34 & 2.235 & 0 & 6 & 1 & 3 & 3 & 4 & 76.62 & 76.62 & 1.2426 & 04 & 3 & 4 & 3 & 12 \\
\hline 44.08 & 44.08 & 2.053 & 1 & 5 & 1 & 8 & 8 & 11 & 78.16 & 78.16 & 1.2220 & 11 & 3 & 6 & 5 & 19 \\
\hline 44.34 & 44.34 & 2.041 & 0 & 8 & 0 & 16 & 16 & 23 & 79.44 & 79.44 & 1.2054 & 210 & 0 & 2 & 2 & 7 \\
\hline 46.36 & 46.46 & 1.9568 & 0 & 0 & 2 & 26 & 36 & 40 & 80.23 & 80.24 & 1.1955 & 13 & 3 & 1 & 1 & 3 \\
\hline 46.45 & & 1.9535 & 1 & 7 & 0 & 22 & & 33 & 80.85 & 80.90 & 1.1878 & 31 & 0 & 0 & 1 & 0 \\
\hline 50.38 & 50.38 & 1.8100 & 0 & 8 & 1 & 8 & 8 & 14 & 80.91 & & 1.1872 & 26 & 2 & 1 & & 4 \\
\hline 51.08 & 51.08 & 1.7865 & 2 & 0 & 0 & 19 & 17 & 32 & 81.08 & 81.14 & 1.1851 & 113 & 0 & 0 & 1 & 1 \\
\hline 51.77 & 51.76 & 1.7646 & 0 & 4 & 2 & 3 & 3 & 5 & 81.81 & 81.80 & 1.1764 & 06 & 3 & 0 & 0 & 1 \\
\hline 52.30 & 52.30 & 1.7478 & 1 & 7 & 1 & 22 & 20 & 39 & 82.92 & 82.92 & 1.1635 & 33 & 0 & 3 & 2 & 9 \\
\hline 53.65 & 53.66 & 1.7069 & 1 & 1 & 2 & 2 & 2 & 3 & 83.93 & 83.94 & 1.1520 & 210 & 1 & 0 & 0 & 1 \\
\hline 56.15 & 56.14 & 1.6368 & 1 & 3 & 2 & 19 & 19 & 37 & 84.35 & 84.34 & 1.1473 & 15 & 3 & 1 & 1 & 3 \\
\hline 56.15 & & 1.6367 & 2 & 4 & 0 & 2 & & 4 & 85.33 & 85.32 & 1.1366 & 31 & 1 & 5 & 4 & 17 \\
\hline 56.29 & 56.30 & 1.6331 & 01 & 10 & 0 & 4 & 15 & 8 & 85.55 & 85.58 & 1.1343 & 113 & 1 & 1 & 3 & 3 \\
\hline 56.86 & & 1.6179 & 1 & 9 & 0 & 0 & & 0 & 86.63 & 86.64 & 1.1228 & 111 & 2 & 1 & 1 & 5 \\
\hline 57.80 & 57.80 & 1.5939 & 2 & 2 & 1 & 1 & 2 & 3 & 87.01 & 87.14 & 1.1189 & 35 & 0 & 1 & 3 & 3 \\
\hline 58.00 & 58.00 & 1.5888 & 0 & 6 & 2 & 2 & 2 & 4 & 87.11 & & 1.1179 & 014 & 1 & 2 & & 8 \\
\hline 60.93 & 60.94 & 1.5193 & 1 & 5 & 2 & 5 & 5 & 11 & 87.17 & & 1.1173 & 012 & 2 & 2 & & 7 \\
\hline 61.35 & 61.34 & 1.5100 & 2 & 4 & 1 & 17 & 15 & 37 & 87.37 & 87.38 & 1.1152 & 33 & 1 & 1 & 2 & 3 \\
\hline 61.47 & & 1.5072 & 01 & 10 & 1 & 0 & & 1 & 88.08 & 88.08 & 1.1081 & 28 & 2 & 5 & 4 & 19 \\
\hline 62.02 & 62.08 & 1.4952 & 1 & 9 & 1 & 1 & 2 & 3 & 88.97 & 88.98 & 1.0993 & 08 & 3 & 1 & 1 & 5 \\
\hline 62.10 & & 1.4935 & 2 & 6 & 0 & 1 & & 3 & & & & & & & & \\
\hline 66.09 & 66.10 & 1.4126 & 0 & 8 & 2 & 7 & 6 & 18 & & & & & & & & \\
\hline 67.01 & 67.02 & 1.3954 & 2 & 6 & 1 & 1 & 1 & 3 & & & & & & & & \\
\hline 67.72 & 67.72 & 1.3825 & 1 & 7 & 2 & 11 & 9 & 29 & & & & & & & & \\
\hline 68.37 & 68.36 & 1.3710 & 1 & 11 & 0 & 1 & 1 & 4 & & & & & & & & \\
\hline
\end{tabular}


Table D.2. Simulated X-ray powder pattern for $\mathrm{Sr}_{0.9} \mathrm{Ca}_{0.1} \mathrm{CuO}_{2}$

\begin{tabular}{|c|c|c|c|c|c|c|c|c|c|c|c|c|c|c|c|c|}
\hline $2 \theta$ & PEAK & $d$ & $h$ & $k$ & $\operatorname{l1} 11$ & $\mid N T) \mid$ & $(\mathrm{PK}) \mathrm{I}$ & (DS) & $2 \theta$ & PEAK & $\bar{d}$ & $h k$ & & (NT) & $(\mathrm{PK})$ & I(DS) \\
\hline 10.85 & 10.86 & 8.146 & 0 & 2 & 0 & 1 & 1 & 0 & 70.30 & 70.30 & 1.3379 & 28 & 0 & 6 & 5 & 16 \\
\hline 21.80 & 21.82 & 4.073 & 0 & 4 & 0 & 16 & 18 & 9 & 71.82 & 71.82 & 1.3133 & 20 & 2 & 10 & 9 & 29 \\
\hline 25.27 & 25.28 & 3.522 & 0 & 2 & 1 & 6 & 7 & 4 & 73.32 & 73.32 & 1.2901 & 111 & 9 & 12 & 10 & 35 \\
\hline 25.67 & 25.68 & 3.468 & 1 & 1 & 0 & 18 & 20 & 12 & 73.63 & 73.54 & 1.2855 & 02 & 3 & 0 & 6 & 1 \\
\hline 30.05 & 30.06 & 2.971 & 1 & 3 & 0 & 62 & 65 & 52 & 73.84 & 73.84 & 1.2824 & 012 & 1 & 1 & 1 & 2 \\
\hline 31.72 & 31.72 & 2.819 & 0 & 4 & 1 & 56 & 58 & 50 & 74.98 & 74.98 & 1.2657 & 28 & 1 & 4 & 4 & 12 \\
\hline 32.96 & 32.96 & 2.715 & 0 & 6 & 0 & 5 & 5 & 5 & 76.01 & 76.02 & 1.2510 & 010 & 2 & 3 & 3 & 8 \\
\hline 34.56 & 34.56 & 2.593 & 1 & 1 & 11 & 001 & 100 & 100 & 76.09 & & 1.2499 & 24 & 2 & 1 & & 4 \\
\hline 37.44 & 37.44 & 2.400 & 1 & 5 & 0 & 11 & 12 & 13 & 76.56 & 76.80 & 1.2434 & 19 & 2 & 0 & 3 & 0 \\
\hline 38.02 & 38.02 & 2.365 & 1 & 3 & 1 & 12 & 12 & 14 & 76.81 & & 1.2400 & 04 & 3 & 4 & & 12 \\
\hline 40.43 & 40.44 & 2.229 & 0 & 6 & 1 & 4 & 4 & 5 & 78.40 & 78.40 & 1.2188 & 11 & 3 & 6 & 5 & 19 \\
\hline 44.26 & 44.26 & 2.045 & 1 & 5 & 1 & 7 & 8 & 9 & 79.86 & 79.86 & 1.2001 & 210 & 0 & 2 & 2 & 7 \\
\hline 44.45 & 44.46 & 2.036 & 0 & 8 & 0 & 17 & 17 & 24 & 80.48 & 80.48 & 1.1924 & 13 & 3 & 1 & 1 & 4 \\
\hline 46.47 & 46.48 & 1.9527 & 0 & 0 & 2 & 27 & 28 & 40 & 81.32 & 81.32 & 1.1823 & 26 & 2 & 1 & 1 & 4 \\
\hline 46.63 & 46.62 & 1.9463 & 1 & 7 & 0 & 23 & 33 & 34 & 81.36 & & 1.1817 & 113 & 0 & 0 & & 0 \\
\hline 50.50 & 50.50 & 1.8057 & 0 & 8 & 1 & 8 & 8 & 13 & 81.51 & 81.56 & 1.1800 & 31 & 0 & 0 & 1 & 0 \\
\hline 51.45 & 51.46 & 1.7746 & 2 & 0 & 0 & 19 & 17 & 32 & 82.02 & 82.02 & 1.1739 & 06 & 3 & 0 & 0 & 1 \\
\hline 51.88 & 51.88 & 1.7608 & 0 & 4 & 2 & 3 & 3 & 5 & 83.58 & 83.58 & 1.1560 & 33 & 0 & 2 & 2 & 9 \\
\hline 52.49 & 52.50 & 1.7419 & 1 & 7 & 1 & 22 & 19 & 38 & 84.36 & 84.36 & 1.1472 & 210 & 1 & 0 & 0 & 1 \\
\hline 53.84 & 53.84 & 1.7015 & 1 & 1 & 2 & 2 & 2 & 4 & 84.62 & 84.62 & 1.1443 & 15 & 3 & 1 & 1 & 2 \\
\hline 56.33 & 56.34 & 1.6218 & 1 & 3 & 2 & 18 & 18 & 36 & 85.85 & 86.00 & 1.1311 & 113 & 1 & 1 & 4 & 2 \\
\hline 56.43 & & 1.6292 & 01 & 10 & 0 & 4 & & 8 & 85.99 & & 1.1295 & 31 & 1 & 4 & & 16 \\
\hline 56.52 & 56.48 & 1.6269 & 2 & 4 & 0 & 2 & 14 & 4 & 86.93 & 86.92 & 1.1198 & 111 & 2 & 1 & 1 & 5 \\
\hline 58.14 & 58.16 & 1.5853 & 0 & 6 & 2 & 2 & 2 & 3 & 87.37 & 87.40 & 1.1152 & 014 & 1 & 2 & 3 & 8 \\
\hline 58.16 & & 1.5848 & 2 & 2 & 1 & 1 & & 2 & 87.42 & & 1.1147 & 012 & 2 & 2 & & 7 \\
\hline 61.13 & 61.14 & 1.5148 & 1 & 5 & 2 & 5 & 4 & 11 & 87.69 & 87.68 & 1.1120 & 35 & 0 & 1 & 2 & 3 \\
\hline 61.63 & 61.72 & 1.5036 & 01 & 10 & 1 & 0 & 15 & 0 & 88.05 & 88.04 & 1.1084 & 33 & 1 & 1 & 1 & 3 \\
\hline 61.72 & & 1.5018 & 2 & 4 & 1 & 16 & & 37 & 88.52 & 88.52 & 1.1037 & 28 & 2 & 5 & 4 & 20 \\
\hline 62.24 & 62.24 & 1.4905 & 1 & 9 & 1 & 1 & 2 & 3 & 89.22 & 89.22 & 1.0969 & 08 & 3 & 1 & 1 & 5 \\
\hline 62.47 & 62.46 & 1.4855 & 2 & 6 & 0 & 1 & 2 & 3 & & & & & & & & \\
\hline 66.26 & 66.26 & 1.4095 & 0 & 8 & 2 & 7 & 6 & 18 & & & & & & & & \\
\hline 67.39 & 67.40 & 1.3884 & 2 & 6 & 1 & 1 & 1 & 3 & & & & & & & & \\
\hline 67.95 & 67.94 & 1.3785 & 1 & 7 & 2 & 11 & 10 & 29 & & & & & & & & \\
\hline 68.60 & 68.60 & 1.3668 & 11 & 11 & 0 & 1 & 1 & 4 & & & & & & & & \\
\hline 69.13 & 69.14 & 1.3577 & 01 & & 0 & 2 & 2 & 5 & & & & & & & & \\
\hline
\end{tabular}


Table D.3. Simulated X-ray powder pattern for $\mathrm{Sr}_{0.8} \mathrm{Ca}_{0.2} \mathrm{CuO}_{2}$

\begin{tabular}{|c|c|c|c|c|c|c|c|c|c|c|c|c|c|c|c|c|c|}
\hline $2 \theta$ & PEAK & $d$ & $\bar{h}$ & $k$ & $\operatorname{l1} 1$ & ||$N T) \mid$ & $|(\mathrm{PK})|$ & (DS) & $2 \theta$ & $\overline{\text { PEAK }}$ & $d$ & $\bar{h}$ & $\bar{k}$ & 11 & $\overline{\text { NT) }}$ & $\overline{(P K)}$ & (DS) \\
\hline 10.88 & 10.88 & 8.126 & 0 & 2 & 0 & 2 & 3 & 1 & 70.70 & 70.70 & 1.3314 & 2 & 8 & 0 & 6 & 5 & 16 \\
\hline 21.86 & 21.86 & 4.063 & 0 & 4 & 0 & 16 & 18 & 9 & 72.21 & 72.20 & 1.3072 & 2 & 0 & 2 & 11 & 9 & 30 \\
\hline 25.32 & 25.34 & 3.514 & 0 & 2 & 1 & 5 & 6 & 3 & 73.57 & 73.58 & 1.2863 & 11 & 1 & 1 & 12 & 10 & 35 \\
\hline 25.84 & 25.84 & 3.445 & 1 & 1 & 0 & 21 & 23 & 15 & 73.81 & 73.78 & 1.2828 & 0 & 2 & 3 & 0 & 6 & 1 \\
\hline 30.22 & 30.22 & 2.955 & 1 & 3 & 0 & 61 & 63 & 51 & 74.04 & 74.02 & 1.2793 & 01 & 2 & 1 & 1 & 1 & 2 \\
\hline 31.79 & 31.80 & 2.813 & 0 & 4 & 1 & 56 & 59 & 51 & 75.38 & 75.38 & 1.2599 & 2 & 8 & 1 & 4 & 3 & 12 \\
\hline 33.04 & 33.04 & 2.709 & 0 & 6 & 0 & 4 & 5 & 4 & 76.22 & 76.22 & 1.2481 & 01 & 0 & 2 & 3 & 2 & 8 \\
\hline 34.73 & 34.74 & 2.581 & 1 & 1 & 11 & 100 & 100 & 100 & 76.49 & 76.46 & 1.2444 & 2 & 4 & 2 & 1 & 2 & 4 \\
\hline 37.61 & 37.62 & 2.390 & 1 & 5 & 0 & 11 & 12 & 13 & 77.00 & 77.00 & 1.2374 & 0 & 4 & 3 & 4 & 4 & 12 \\
\hline 38.19 & 38.20 & 2.355 & 1 & 3 & 1 & 13 & 13 & 15 & 78.65 & 78.64 & 1.2156 & 1 & 1 & 3 & 6 & 5 & 19 \\
\hline 40.52 & 40.52 & 2.224 & 0 & 6 & 1 & 4 & 4 & 5 & 80.28 & 80.28 & 1.1949 & 21 & 0 & 0 & 2 & 2 & 7 \\
\hline 44.43 & 44.56 & 2.037 & 1 & 5 & 1 & 6 & 20 & 8 & 80.74 & 80.74 & 1.1893 & 1 & 3 & 3 & 1 & 1 & 4 \\
\hline 44.56 & & 2.032 & 0 & 8 & 0 & 18 & & 25 & 81.65 & 81.74 & 1.1783 & 11 & 3 & 0 & 0 & 1 & 0 \\
\hline 46.57 & 46.58 & 1.9487 & 0 & 0 & 2 & 28 & 27 & 41 & 81.73 & & 1.1773 & 2 & 6 & 2 & 1 & & 3 \\
\hline 46.81 & 46.82 & 1.9390 & 1 & 7 & 0 & 23 & 26 & 35 & 82.18 & 82.22 & 1.1721 & 3 & 1 & 0 & 0 & 0 & 1 \\
\hline 50.63 & 50.64 & 1.8015 & 0 & 8 & 1 & 8 & 7 & 13 & 82.24 & & 1.1714 & 0 & 6 & 3 & 0 & & 1 \\
\hline 51.83 & 51.82 & 1.7627 & 2 & 0 & 0 & 19 & 18 & 32 & 84.25 & 84.24 & $1.148 \mathrm{~A}$ & 3 & 3 & 0 & 2 & 2 & 8 \\
\hline 52.00 & 51.96 & 1.7571 & 0 & 4 & 2 & 3 & 13 & 4 & 84.80 & 84.88 & 1.1424 & & 0 & 1 & 0 & 1 & 1 \\
\hline 52.68 & 52.68 & 1.7360 & 1 & 7 & 1 & 21 & 20 & 38 & 84.89 & & 1.1414 & 1 & 5 & 3 & 1 & & 2 \\
\hline 54.02 & 54.02 & 1.6962 & 1 & 1 & 2 & 3 & 3 & 5 & 86.15 & 86.16 & 1.1279 & 11 & 3 & 1 & 1 & 1 & 2 \\
\hline 56.53 & 56.54 & 1.6268 & 1 & 3 & 2 & 18 & 18 & 35 & 86.67 & 86.68 & 1.1224 & 3 & 1 & 1 & 4 & 4 & 16 \\
\hline 56.58 & & 1.6252 & 0 & 10 & 0 & 4 & & 8 & 87.23 & 87.22 & 1.1167 & 11 & 1 & 2 & 1 & 1 & 5 \\
\hline 56.89 & 56.88 & 1.0171 & 2 & 4 & 0 & 2 & 3 & 4 & 87.63 & 87.66 & 1.1126 & 01 & 4 & 1 & 2 & 3 & 8 \\
\hline 58.28 & 58.28 & 1.5819 & 0 & 6 & 2 & 1 & 1 & 3 & 87.68 & & 1.1121 & 01 & 2 & 2 & 2 & & 7 \\
\hline 58.54 & 58.52 & 1.5756 & 2 & 2 & 1 & 1 & 1 & 2 & 88.38 & 88.38 & 1.1051 & 3 & 5 & 0 & 1 & 1 & 3 \\
\hline 61.34 & 61.34 & 1.5102 & 1 & 5 & 2 & 5 & 4 & 11 & 88.73 & 88.74 & 1.1016 & 3 & 3 & 1 & 1 & 1 & 3 \\
\hline 61.80 & 62.10 & 1.5000 & 0 & 10 & 1 & 0 & 15 & 0 & 88.97 & 88.96 & 1.0993 & 2 & 8 & 2 & 5 & 5 & 20 \\
\hline 62.09 & & 1.4936 & 2 & 4 & 1 & 16 & & 37 & 89.47 & 89.46 & 1.0945 & 0 & 8 & 3 & 1 & 1 & 5 \\
\hline 62.45 & 62.26 & 1.4858 & 1 & 9 & 1 & 1 & 9 & 3 & & & & & & & & & \\
\hline 62.85 & 62.84 & 1.4774 & 2 & 6 & 0 & 1 & 1 & 2 & & & & & & & & & \\
\hline 66.42 & 66.42 & 1.4063 & 0 & 8 & 2 & 8 & 7 & 19 & & & & & & & & & \\
\hline 67.78 & 67.78 & 1.3815 & 2 & 6 & 1 & 1 & 2 & 4 & & & & & & & & & \\
\hline 68.17 & 68.18 & 1.3745 & 1 & 7 & 2 & 12 & 10 & 30 & & & & & & & & & \\
\hline 68.85 & 58.84 & 1.3627 & 1 & & 0 & 1 & 1 & 3 & & & & & & & & & \\
\hline 69.33 & 69.32 & 1.3544 & 0 & 12 & 0 & 2 & 2 & 5 & & & & & & & & & \\
\hline
\end{tabular}


Table D.4. Simulated X-ray powder pattern for $\mathrm{Sr}_{0.7} \mathrm{Ca}_{0.3} \mathrm{CuO}_{2}$

\begin{tabular}{|c|c|c|c|c|c|c|c|c|c|c|c|c|c|c|c|c|c|}
\hline \multirow{2}{*}{$\frac{2 \theta}{10.91}$} & \multirow{2}{*}{$\frac{\text { PEAK }}{10.92}$} & \multirow{2}{*}{$\frac{d}{8.106}$} & \multirow{2}{*}{$\frac{h}{0}$} & \multirow{2}{*}{$\frac{k}{2}$} & \multicolumn{4}{|c|}{$\ell|(\mid N T)|(P K) \mid(D S)$} & \multirow{2}{*}{$\frac{2 \theta}{71.10}$} & \multirow{2}{*}{$\frac{\text { PEAK }}{71.10}$} & \multirow{2}{*}{$\frac{d}{1.3249}$} & \multirow{2}{*}{$\frac{h}{2}$} & \multirow[t]{2}{*}{ k } & \multicolumn{4}{|c|}{$\ell|(I N T)|(P K) \mid(D S)$} \\
\hline & & & & & 0 & 4 & 5 & 1 & & & & & & 0 & 6 & 5 & 17 \\
\hline 21.91 & 21.92 & 4.053 & 0 & 4 & 0 & 15 & 17 & 9 & 72.60 & 72.60 & 1.3011 & 2 & 0 & 2 & 11 & 9 & 30 \\
\hline 25.38 & 25.38 & 3.507 & 0 & 2 & 1 & 4 & 4 & 2 & 73.83 & 73.84 & 1.2825 & 11 & 1 & 1 & 12 & 10 & 35 \\
\hline 26.01 & 26.02 & 3.423 & 1 & 1 & 0 & 25 & 27 & 17 & 73.99 & 74.04 & 1.2801 & 0 & 2 & 3 & 0 & 6 & 1 \\
\hline 30.39 & 30.40 & 2.939 & 1 & 3 & 0 & 60 & 62 & 50 & 74.25 & & 1.2763 & 01 & 2 & 1 & 1 & & 2 \\
\hline 31.86 & 31.86 & 2.806 & 0 & 4 & 1 & 57 & 57 & 51 & 75.79 & 75.80 & 1.2541 & 2 & 8 & 1 & 4 & 3 & 11 \\
\hline 33.13 & 33.14 & 2.702 & 0 & 6 & 0 & 4 & 4 & 3 & 76.43 & 76.42 & 1.2453 & 01 & 0 & 2 & 3 & 2 & 8 \\
\hline 34.89 & 34.90 & 2.569 & 1 & 1 & 11 & 100 & 100 & 100 & 76.89 & 76.90 & 1.2389 & 2 & 4 & 2 & 1 & 1 & 4 \\
\hline 37.78 & 37.78 & 2.379 & 1 & 5 & 0 & 11 & 11 & 13 & 77.19 & 77.20 & 1.2348 & 0 & 4 & 3 & 4 & 4 & 12 \\
\hline 38.36 & 38.36 & 2.345 & 1 & 3 & 1 & 14 & 14 & 16 & 78.90 & 78.90 & 1.2124 & 1 & 1 & 3 & 6 & 5 & 18 \\
\hline 40.62 & 40.62 & 2.219 & 0 & 6 & 1 & 4 & 4 & 5 & 80.71 & 80.72 & 1.1896 & 21 & 0 & 0 & 2 & 2 & 7 \\
\hline 44.61 & 44.68 & 2.030 & 1 & 5 & 1 & 5 & 21 & 7 & 81.00 & 80.98 & 1.1861 & 1 & 3 & 3 & 1 & 2 & 4 \\
\hline 44.68 & & 2.027 & 0 & 8 & 0 & 18 & & 26 & 82.15 & 82.16 & 1.1723 & 2 & 6 & 2 & 1 & 1 & 3 \\
\hline 46.67 & 46.68 & 1.9446 & 0 & 0 & 2 & 28 & 27 & 42 & 82.45 & 82.42 & 1.1689 & 0 & 6 & 3 & 0 & 1 & 1 \\
\hline 47.00 & 47.00 & 1.9318 & 1 & 7 & 0 & 24 & 24 & 36 & 82.85 & 82.84 & 1.1642 & 3 & 1 & 0 & 0 & 0 & 1 \\
\hline 48.08 & 48.08 & 1.8910 & 0 & 2 & 2 & 0 & 0 & 0 & 84.93 & 84.94 & 1.1409 & 3 & 3 & 0 & 2 & 2 & 8 \\
\hline 50.76 & 50.76 & 1.7972 & 0 & 8 & 1 & 8 & 7 & 13 & 85.17 & 85.18 & 1.1384 & 1 & 5 & 3 & 0 & 1 & 2 \\
\hline 52.12 & 52.20 & 1.7533 & 0 & 4 & 2 & 2 & 19 & 4 & 85.24 & & 1.1376 & 21 & 10 & 1 & 0 & & 0 \\
\hline 52.20 & & 1.7508 & 2 & 0 & 0 & 19 & & 33 & 86.46 & 86.46 & 1.1247 & 11 & 13 & 1 & 0 & 0 & 2 \\
\hline 52.88 & 52.88 & 1.7301 & 1. & 7 & 1 & 21 & 19 & 37 & 87.37 & 87.36 & 1.1153 & 3 & 1 & 1 & 4 & 4 & 16 \\
\hline 54.21 & 54.20 & 1.6908 & 1 & 1 & 2 & 3 & 3 & 6 & 87.53 & 87.54 & 1.1136 & 11 & 11 & 2 & 1 & 2 & 4 \\
\hline 56.72 & 56.72 & 1.6217 & 1 & 3 & 2 & 18 & 19 & 34 & 87.90 & 87.92 & 1.1099 & 01 & 14 & 1 & 2 & 3 & 8 \\
\hline 56.73 & & 1.6213 & 01 & 10 & 0 & 4 & & 8 & 87.93 & & 1.1096 & 01 & 12 & 2 & 2 & & 6 \\
\hline 57.27 & 57.28 & 1.6073 & 2 & 4 & 0 & 2 & 2 & 3 & 89.08 & 89.08 & 1.0982 & 3 & 5 & 0 & 1 & 1 & 3 \\
\hline 58.42 & 58.42 & 1.5784 & 0 & 6 & 2 & 1 & 1 & 2 & 89.42 & 89.42 & 1.0949 & 2 & 8 & 2 & 5 & 5 & 21 \\
\hline 58.91 & 58.92 & 1.5664 & 2 & 2 & 1 & 1 & 1 & 1 & 89.44 & & 1.0948 & 3 & 3 & 1 & 1 & & 4 \\
\hline 61.54 & 61.54 & 1.5057 & 1 & 5 & 2 & 5 & 4 & 11 & 89.72 & 89.70 & 1.0921 & 0 & 8 & 3 & 1 & 4 & 4 \\
\hline 62.47 & 62.48 & 1.4854 & 2 & 4 & 1 & 16 & 14 & 36 & & & & & & & & & \\
\hline 62.67 & 62.64 & 1.4812 & 1 & 9 & 1 & 2 & 9 & 4 & & & & & & & & & \\
\hline 63.24 & 63.24 & 1.4693 & 2 & 6 & 0 & 1 & 1 & 2 & & & & & & & & & \\
\hline 66.59 & 66.60 & 1.4031 & 0 & 8 & 2 & 8 & 7 & 20 & & & & & & & & & \\
\hline 68.17 & 68.18 & 1.3745 & 2 & 6 & 1 & 2 & 2 & 4 & & & & & & & & & \\
\hline 68.40 & 68.40 & 1.3705 & 1 & 7 & 2 & 12 & 11 & 30 & & & & & & & & & \\
\hline 69.09 & 69.08 & 1.3585 & 11 & 11 & 0 & 1 & 1 & 3 & & & & & & & & & \\
\hline 69.52 & 69.52 & 1.3511 & 01 & 12 & 0 & 2 & 2 & 5 & & & & & & & & & \\
\hline
\end{tabular}


Table D.5. Simulated X-ray powder pattern for $\mathrm{Sr}_{(0.6} \mathrm{Ca}_{0.4} \mathrm{CuO}_{2}$.

\begin{tabular}{|c|c|c|c|c|c|c|c|c|c|c|c|c|c|c|c|c|}
\hline $2 \theta$ & PEAK & d & $h$ & $k$ & 811 & |NT)| & $|(\mathrm{PK})|$ & $(D S)$ & $2 \theta$ & PEAK & $d$ & $h k$ & & & & (DS) \\
\hline 10.93 & 10.94 & 8.087 & 0 & 2 & 0 & 6 & 8 & 2 & 69.71 & 69.72 & 1.3478 & 012 & 0 & 2 & 2 & 4 \\
\hline 21.97 & 2.1 .98 & 4.043 & 0 & 4 & 0 & 15 & 17 & 9 & 71.51 & 71.50 & 1.3183 & 28 & 0 & 6 & 5 & 17 \\
\hline 25.44 & 25.44 & 3.499 & 0 & 2 & 1 & 2 & 3 & 2 & 73.00 & 73.00 & 1.2950 & 20 & 2 & 11 & 9 & 30 \\
\hline 26.19 & 26.20 & 3.400 & 1 & 1 & 0 & 30 & 32 & 21 & 74.09 & 74.08 & 1.2787 & 111 & 1 & 12 & 10 & 35 \\
\hline 30.56 & 30.58 & 2.922 & 1 & 3 & 0 & 58 & 59 & 49 & 74.17 & & 1.2775 & 02 & 3 & 0 & & 0 \\
\hline 31.94 & 31.94 & 2.800 & 0 & 4 & 1 & 57 & 58 & 51 & 74.46 & 74.30 & 1.2732 & 012 & 1 & 1 & 6 & 2 \\
\hline 33.21 & 33.22 & 2.696 & 0 & 6 & 0 & 3 & 3 & 3 & 76.21 & 76.20 & 1.2483 & 28 & 1 & 4 & 3 & 11 \\
\hline 35.06 & 35.06 & 2.557 & 1 & 1 & 11 & 100 & 100 & 100 & 76.63 & 76.64 & 1.2424 & 010 & 2 & 3 & 3 & 8 \\
\hline 37.96 & 37.96 & 2.369 & 1 & 5 & 0 & 11 & 11 & 13 & 77.30 & 77.38 & 1.2333 & 24 & 2 & 1 & 4 & 4 \\
\hline 38.53 & 38.54 & 2.335 & 1 & 3 & 1 & 15 & 15 & 17 & 77.39 & & 1.2322 & 04 & 3 & 4 & & 12 \\
\hline 40.72 & 40.72 & 2.214 & 0 & 6 & 1 & 5 & 5 & 6 & 79.15 & 79.14 & 1.2091 & 11 & 3 & 6 & 5 & 18 \\
\hline 44.79 & 44.80 & 2.022 & 1 & 5 & 1 & 4 & 22 & 6 & 81.15 & 81.16 & 1.1843 & 210 & 0 & 2 & 2 & 7 \\
\hline 44.79 & & 2.022 & 0 & 8 & 0 & 19 & & 27 & 81.26 & 81.24 & 1.1830 & 13 & 3 & 1 & 2 & 5 \\
\hline 46.77 & 46.78 & 1.9406 & 0 & 0 & 2 & 29 & 28 & 43 & 82.58 & 82.60 & 1.1673 & 26 & 2 & 1 & 1 & 2 \\
\hline 47.19 & 47.20 & 1.9245 & 1 & 7 & 0 & 25 & 24 & 37 & 82.67 & & 1.1663 & 06 & 3 & 0 & & 1 \\
\hline 48.19 & 48.18 & 1.8870 & 0 & 2 & 2 & 0 & 0 & 0 & 83.55 & 83.54 & 1.1563 & 31 & 0 & 0 & 0 & 1 \\
\hline 50.89 & 50.88 & 1.7930 & 0 & 8 & 1 & 7 & 7 & 12 & 85.45 & 85.64 & 1.1354 & 15 & 3 & 0 & 2 & 1 \\
\hline 52.25 & 52.26 & 1.7495 & 0 & 4 & 2 & 2 & 3 & 4 & 85.63 & & 1.1334 & 33 & 0 & 2 & & 8 \\
\hline 52.59 & 52.60 & 1.7389 & 2 & 0 & 0 & 19 & 18 & 33 & 86.77 & 86.76 & 1.1214 & 113 & 1 & 0 & 0 & 1 \\
\hline 53.07 & 53.08 & 1.7242 & 1 & 7 & 1 & 21 & 19 & 36 & 87.83 & 87.84 & 1.1106 & 111 & 2 & 1 & 1 & 4 \\
\hline 53.89 & 53.88 & 1.7000 & 2 & 2 & 0 & 0 & 0 & 0 & 88.07 & 88.08 & 1.1082 & 31 & 1 & 4 & 5 & 15 \\
\hline 54.39 & 54.40 & 1.6854 & 1 & 1 & 2 & 4 & 4 & 8 & 88.17 & & 1.1072 & 014 & 1 & 2 & & 8 \\
\hline 56.88 & 56.90 & 1.6173 & 0 & 10 & 0 & 4 & 18 & 8 & 88.19 & & 1.1070 & 012 & 2 & 2 & & 6 \\
\hline 56.91 & & 1.6166 & 1 & 3 & 2 & 17 & & 33 & 89.80 & 89.88 & 1.0913 & 35 & 0 & 1 & 5 & 3 \\
\hline 57.66 & 57.66 & 1.5974 & 2 & 4 & 0 & 2 & 2 & 3 & 89.88 & & 1.0905 & 28 & 2 & 6 & & 21 \\
\hline 58.56 & 58.56 & 1.5749 & 0 & 6 & 2 & 1 & 1 & 2 & 89.97 & & 1.0897 & 08 & 3 & 1 & & 4 \\
\hline 59.30 & 59.30 & 1.5572 & 2 & 2 & 1 & 0 & 0 & 1 & & & & & & & & \\
\hline 61.75 & 61.76 & 1.5011 & 1 & 5 & 2 & 5 & 4 & 11 & & & & & & & & \\
\hline 62.86 & 62.86 & 1.4772 & 2 & 4 & 1 & 16 & 15 & 36 & & & & & & & & \\
\hline 62.89 & & 1.4765 & 1 & 9 & 1 & 2 & & 4 & & & & & & & & \\
\hline 63.63 & 63.62 & 1.4612 & 2 & 6 & 0 & 1 & 1 & 2 & & & & & & & & \\
\hline 66.76 & 66.76 & 1.4000 & 0 & 8 & 2 & 8 & 7 & 20 & & & & & & & & \\
\hline 68.57 & 68.62 & 1.3675 & 2 & 6 & 1 & 2 & 11 & 4 & & & & & & & & \\
\hline 68.63 & & 1.3665 & 1 & 7 & 2 & 12 & & 31 & & & & & & & & \\
\hline 69.33 & 69.34 & 1.3543 & 1 & 11 & 0 & 1 & 1 & 3 & & & & & & & & \\
\hline
\end{tabular}


Table D.6. Simulated $\mathrm{X}$-ray powder pattern for $\mathrm{Sr}_{0.5} \mathrm{Ca}_{0.5} \mathrm{CuO}_{2}$

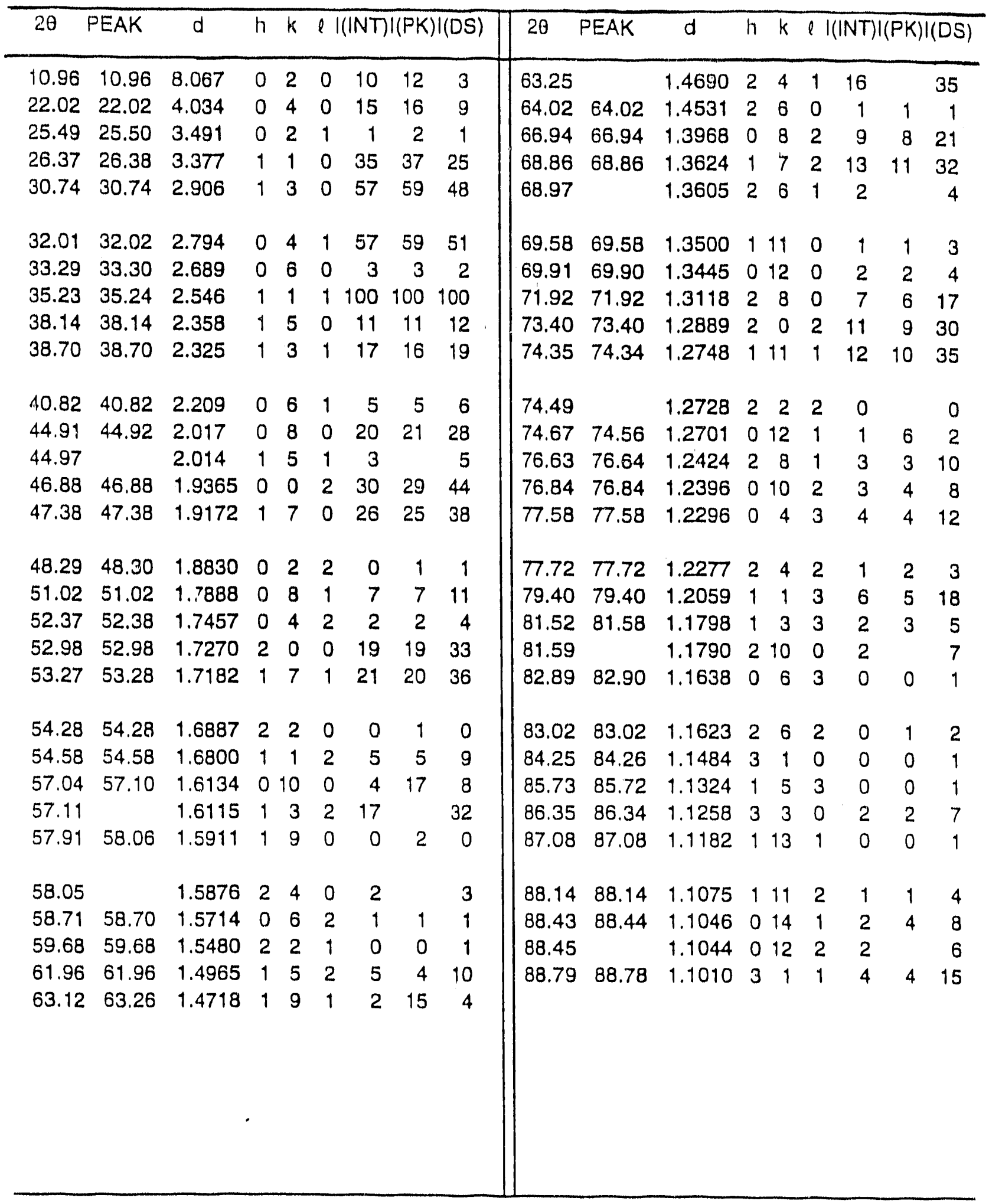


Table D.7. Simulated X-ray powder pattern for $\mathrm{Sr}_{(, .4} \mathrm{Ca}_{(, 6} \mathrm{CuO}_{2}$

\begin{tabular}{|c|c|c|c|c|c|c|c|c|c|c|c|c|c|c|c|c|c|}
\hline $2 \theta$ & PEAK & $d$ & $n$ & $k$ & 811 & |NT) & (PK) & (IDS) & $2 \theta$ & PEAK & d & $\mathrm{h}$ & $k$ & $\operatorname{ll}$ & |NT)| & (PK) & I(DS) \\
\hline 10.99 & 11.00 & 8.047 & 0 & 2 & 0 & 14 & 17 & 5 & 63.65 & 63.66 & 1.4607 & 2 & 4 & 1 & 16 & 14 & 35 \\
\hline 22.07 & 22.08 & 4.024 & 0 & 4 & 0 & 14 & 16 & 8 & 64.43 & 64.42 & 1.4450 & 2 & 6 & 0 & 0 & 0 & 1 \\
\hline 25.55 & 25.56 & 3.484 & 0 & 2 & 1 & 1 & 1 & 0 & 67.11 & 67.10 & 1.3937 & 0 & 8 & 2 & 9 & 8 & 22 \\
\hline 26.55 & 26.56 & 3.355 & 1 & 1 & 0 & 41 & 44 & 29 & 69.09 & 69.10 & 1.3584 & 1 & 7 & 2 & 13 & 11 & 33 \\
\hline 30.92 & 30.92 & 2.890 & 1 & 3 & 0 & 55 & 57 & 47 & 69.38 & 69.28 & 1.3535 & 2 & 6 & 1 & 2 & 7 & 5 \\
\hline 32.09 & 32.10 & 2.787 & 0 & 4 & 1 & 57 & 59 & 51 & 69.83 & 69.84 & 1.3458 & 11 & 11 & 0 & 1 & 1 & 3 \\
\hline 33.38 & 33.38 & 2.682 & 0 & 6 & 0 & 2 & 2 & 2 & 70.11 & 70.10 & 1.3412 & 01 & 12 & 0 & 2 & 2 & 4 \\
\hline 35.40 & 35.40 & 2.534 & 1 & 1 & 11 & 100 & 100 & 100 & 72.34 & 72.34 & 1.3052 & 2 & 8 & 0 & 7 & 6 & 18 \\
\hline 38.32 & 38.32 & 2.347 & 1 & 5 & 0 & 11 & 11 & 12 & 73.81 & 73.82 & 1.2827 & 2 & 0 & 2 & 11 & 9 & 30 \\
\hline 38.88 & 38.88 & 2.314 & 1 & 3 & 1 & 18 & 18 & 20 & 74.61 & 74.62 & 1.2710 & 11 & 11 & 1 & 12 & 11 & 35 \\
\hline 40.92 & 40.92 & 2.204 & 0 & 6 & 1 & 6 & 5 & 7 & 74.88 & 74.84 & 1.2671 & 01 & 12 & 1 & 1 & 6 & 2 \\
\hline 45.03 & 45.02 & 2.012 & 0 & 8 & 0 & 22 & 21 & 30 & 74.90 & & 1.2668 & 2 & 2 & 2 & 0 & & 1 \\
\hline 45.16 & 45.14 & 2.006 & 1 & 5 & 1 & 3 & 16 & 3 & 77.05 & 77.06 & 1.2367 & 01 & 10 & 2 & 3 & 5 & 8 \\
\hline 46.98 & 46.98 & 1.9324 & 0 & 0 & 2 & 31 & 30 & 45 & 77.06 & & 1.2366 & 2 & 8 & 1 & 3 & & 9 \\
\hline 47.57 & 47.58 & 1.9099 & 1 & 7 & 0 & 27 & 26 & 40 & 77.78 & 77.78 & 1.2269 & 0 & 4 & 3 & 4 & 4 & 12 \\
\hline 48.40 & 48.40 & 1.8790 & 0 & 2 & 2 & 1 & 1 & 1 & 78.14 & 78.12 & 1.2221 & 2 & 4 & 2 & 1 & 1 & 3 \\
\hline 51.15 & 51.14 & 1.7845 & 0 & 8 & 1 & 7 & 6 & 11 & 79.66 & 79.66 & 1.2027 & 1 & 1 & 3 & 6 & 5 & 17 \\
\hline 52.49 & 52.50 & 1.7420 & 0 & 4 & 2 & 2 & 2 & 4 & 81.79 & 81.78 & 1.1767 & 1 & 3 & 3 & 2 & 2 & 5 \\
\hline 53.38 & 53.48 & 1.7151 & 2 & 0 & 0 & 19 & 29 & 34 & 82.04 & 82.04 & 1.1736 & 21 & 10 & 0 & 2 & 3 & 7 \\
\hline 53.47 & & 1.7122 & 1 & 7 & 1 & 20 & & 35 & 83.11 & 83.10 & 1.1613 & 0 & 6 & 3 & 0 & 0 & 2 \\
\hline 54.67 & 54.78 & 1.6774 & 2 & 2 & 0 & 0 & 6 & 1 & 83.46 & 83.46 & 1.1572 & 2 & 6 & 2 & 0 & 0 & 1 \\
\hline 54.78 & & 1.6745 & 1 & 1 & 2 & 6 & & 11 & 84.97 & 84.96 & 1.1405 & 3 & 1 & 0 & 0 & 0 & 1 \\
\hline 57.19 & 57.32 & 1.6095 & 01 & 10 & 0 & 5 & 17 & 9 & 86.01 & 86.02 & 1,1294 & 1 & 5 & 3 & 0 & 0 & 1 \\
\hline 57.31 & & 1.6064 & 1 & 3 & 2 & 16 & & 31 & 87.07 & 87.08 & 1.1183 & 3 & 3 & 0 & 2 & 2 & 7 \\
\hline 58.13 & 58.12 & 1.5857 & 1 & 9 & 0 & 0 & 1 & 1 & 87.40 & 87.34 & 1.1150 & 11 & 13 & 1 & 0 & 1 & 1 \\
\hline 58.45 & 58.44 & 1.5777 & 2 & 4 & 0 & 1 & 1 & 3 & 88.45 & 88.46 & 1.1044 & 11 & 11 & 2 & 1 & 1 & 4 \\
\hline 58.85 & 58.84 & 1.5679 & 0 & 6 & 2 & 0 & 1 & 1 & 88.70 & 88.72 & 1.1019 & 01 & 14 & 1 & 2 & 3 & 7 \\
\hline 60.08 & 60.08 & 1.5387 & 2 & 2 & 1 & 0 & 0 & 0 & 88.71 & & 1.1018 & 01 & 12 & 2 & 1 & & 5 \\
\hline 62.17 & 62.18 & 1.4919 & 1 & 5 & 2 & 5 & 4 & 10 & 89.53 & 89.52 & 1.0939 & 3 & 1 & 1 & 4 & 3 & 14 \\
\hline 63.35 & 63.36 & 1.4670 & 1 & 9 & 1 & 2 & 2 & 5 & & & & & & & & & \\
\hline
\end{tabular}


Table D.8. Simulated $X$-ray powder pattern for $\mathrm{Sr}_{(., 3} \mathrm{Ca}_{0.7} \mathrm{CuO}_{2}$

\begin{tabular}{|c|c|c|c|c|c|c|c|c|c|c|c|c|c|c|c|c|c|}
\hline $2 \theta$ & PEAK & d & $h$ & $k$ & & NTII & $P K) \mid($ & DS) & $2 \theta$ & PEAK & $d$ & $h k$ & & II) IN & VT)I & $(\mathrm{PK}) \mathrm{I}$ & (DS) \\
\hline 11.01 & 11.02 & 8.028 & 0 & 2 & 0 & 20 & 25 & 7 & 64.84 & 64.84 & 1.4368 & 26 & 6 & 0 & 0 & 0 & 1 \\
\hline 22.13 & 22.14 & 4.014 & 0 & 4 & 0 & 14 & 15 & 8 & 67.28 & 67.28 & 1.3905 & 08 & 8 & 2 & 10 & 8 & 23 \\
\hline 25.60 & 25.62 & 3.476 & 0 & 2 & 1 & 0 & 0 & 0 & 69.33 & 69.32 & 1.3543 & 17 & 7 & 2 & 14 & 11 & 34 \\
\hline 26.73 & 26.74 & 3.332 & 1 & 1 & 0 & 48 & 52 & 35 & 69.79 & 69.80 & 1.3464 & 26 & 3 & 1. & 2 & 2 & 5 \\
\hline 31.10 & 31.10 & 2.874 & 1 & 3 & 0 & 54 & 55 & 46 & 70.08 & 70.08 & 1.3416 & 111 & & 0 & 1 & 2 & 3 \\
\hline 32.16 & 32.16 & 2.781 & 0 & 4 & 1 & 58 & 59 & 51 & 70.30 & 70.30 & 1.3379 & 012 & & 0 & 1 & 2 & 4 \\
\hline 33.46 & 33.46 & 2.676 & 0 & 6 & 0 & 1 & 2 & 1 & 72.77 & 72.76 & 1.2986 & 28 & 8 & 0 & 7 & 6 & 19 \\
\hline 35.58 & 35.58 & 2.521 & 1 & 1 & 11 & 100 & 100 & 100 & 74.23 & 74.24 & 1.2766 & 20 & 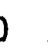 & 2 & 11 & 9 & 30 \\
\hline 38.50 & 38.50 & 2.337 & 1 & 5 & 0 & 11 & 11 & 12 & 74.88 & 74.88 & 1.2671 & 111 & & 1 & 13 & 11 & 35 \\
\hline 39.06 & 39.06 & 2.304 & 1 & 3 & 1 & 20 & 20 & 22 & 75.09 & 75.10 & 1.2640 & 012 & & 1 & 1 & 7 & 3 \\
\hline 41.02 & 41.02 & 2.199 & 0 & 6 & 1 & 6 & 6 & 7 & 75.32 & & 1.2607 & 22 & ? & 2 & 0 & & 1 \\
\hline 45.14 & 45.14 & 2.007 & 0 & 8 & 0 & 23 & 22 & 31 & 77.26 & 77.26 & 1.2338 & 010 & & 2 & 3 & 3 & 9 \\
\hline 45.34 & 45.24 & 1.9984 & 1 & 5 & 1 & 2 & 15 & 2 & 77.50 & 77.50 & 1.2307 & 28 & 3 & 1 & 3 & 4 & 8 \\
\hline 47.09 & 47.10 & 1.9284 & 0 & 0 & 2 & 32 & 30 & 47 & 77.98 & 77.98 & 1.2243 & 04 & 4 & 3 & 4 & 4 & 12 \\
\hline 47.77 & 47.78 & 1.9025 & 1 & 7 & 0 & 28 & 26 & 41 & 78.13 & 78.20 & 1.2223 & 19 & 9 & 2 & 0 & 2 & 1 \\
\hline 48.51 & 48.52 & 1.8750 & 0 & 2 & 2 & 1 & 1 & 1 & 78.57 & 78.58 & 1.2165 & 24 & 4 & 2 & 1 & 1 & 3 \\
\hline 51.28 & 51.28 & 1.7803 & 0 & 8 & 1 & 6 & 6 & 10 & 79.92 & 79.92 & 1.1994 & 11 & 1 & 3 & 6 & 5 & 17 \\
\hline 52.61 & 52.62 & 1.7382 & 0 & 4 & 2 & 2 & 2 & 4 & 82.05 & 82.06 & 1.1735 & 13 & 3 & 3 & 2 & 2 & 6 \\
\hline 53.68 & 53.68 & 1.7062 & 1 & 7 & 1 & 20 & 23 & 34 & 82.50 & 82.50 & 1.1683 & 210 & & 0 & 2 & 2 & 7 \\
\hline 53.78 & 53.78 & 1.7032 & 2 & 0 & 0 & 20 & 28 & 34 & 83.33 & 83.32 & 1.1588 & 06 & 3 & 3 & 1 & 0 & 2 \\
\hline 54.97 & 54.98 & 1.6690 & 1 & 1 & 2 & 8 & 7 & 13 & 83.91 & 83.92 & 1.1522 & 26 & 6 & 2 & 0 & 0 & 1 \\
\hline 55.08 & & 1.6661 & 2 & 2 & 0 & 1 & & 1 & 85.70 & 85.70 & 1.1326 & 31 & 1 & 0 & 1 & 0 & 2 \\
\hline 57.34 & 57.36 & 1.6055 & 01 & 10 & 0 & 5 & 6 & 9 & 86.30 & 86.30 & 1.1264 & 15 & 5 & 3 & 0 & 0 & 0 \\
\hline 57.51 & 57.50 & 1.6013 & 1 & 3 & 2 & 16 & 16 & 30 & 87.71 & 87.82 & 1.1118 & 113 & 3 & 1 & 0 & 2 & 0 \\
\hline 58.34 & 58.34 & 1.5803 & 1 & 9 & 0 & 1 & 1 & 1 & 87.82 & & 1.1107 & 33 & 3 & 0 & 2. & & 7 \\
\hline 58.85 & 58.86 & 1.5 & 2 & 4 & 0 & 1 & 1 & 3 & 88.77 & 88.78 & 1.1013 & 111 & & 2 & 1 & 1 & 4 \\
\hline 58.99 & 59.00 & 1.5645 & 0 & 6 & 2 & 0 & 1 & 1 & 88.97 & 88.98 & 1.0993 & 012 & & 2 & 1 & 3 & 5 \\
\hline 62.39 & 62.38 & 1.4873 & 1 & 5 & 2 & 5 & 4 & 10 & 88.98 & & 1.0992 & 014 & & 1 & 2 & & 7 \\
\hline 63.57 & 63.58 & 1.4623 & 1 & 9 & 1 & 2 & 2 & 5 & & & & & & & & & \\
\hline 64.06 & 64.06 & 1.4524 & 2 & 4 & 1 & 16 & 14 & 35 & & & & & & & & & \\
\hline
\end{tabular}


Table D.9. Simulated X-ray powder pattern for $\mathrm{Sr}_{(0.25} \mathrm{Ca} 0.75 \mathrm{CuO}_{2}$

\begin{tabular}{|c|c|c|c|c|c|c|c|c|c|c|c|c|c|c|c|c|}
\hline $2 \theta$ & PEAK & $d$ & h & $k$ & e IIIN & & $(\mathrm{PK}) \mathrm{I}$ & $\mid(D S)$ & $2 \theta$ & PEAK & $d$ & $h k$ & & (INT) & (PK) & $1(D S)$ \\
\hline 11.03 & 11.04 & 8.018 & 0 & 2 & 02 & 23 & 29 & 8 & 65.05 & 65.04 & 1.4327 & 26 & 0 & 0 & 0 & 0 \\
\hline 22.16 & 22.16 & 4.009 & 0 & 4 & 01 & 4 & 15 & 8 & 67.37 & 67.36 & 1.3889 & 08 & 2 & 10 & 9 & 23 \\
\hline 26.82 & 26.84 & 3.321 & 1 & 1 & 05 & & 57 & 38 & 69.45 & 69.44 & 1.3523 & 17 & 2 & 14 & 12 & 34 \\
\hline 31.19 & 31.20 & 2.865 & 1 & 3 & 05 & 53 & 55 & 45 & 70.00 & 70.00 & 1.3429 & 26 & 1 & 2 & 2 & 5 \\
\hline 32.20 & 32.20 & 2.778 & 0 & 4 & 15 & 88 & 60 & 51 & 70.21 & 70.20 & 1.3395 & 111 & 0 & 1 & 2 & 3 \\
\hline 33.50 & 33.50 & 2.673 & 0 & 6 & 0 & 1 & 1 & 1 & 70.40 & 70.40 & 1.3363 & 012 & 0 & 1 & 2 & 4 \\
\hline 35.66 & 35.66 & 2.515 & 1 & 1 & 110 & 1 & 100 & 100 & 72.98 & 72.98 & 1.2953 & 28 & 0 & 7 & 6 & 19 \\
\hline 38.59 & 38.60 & 2.331 & 1 & 5 & 01 & 11 & 11 & 12 & 74.44 & 74.44 & 1.2735 & 20 & 2 & 11 & 10 & 30 \\
\hline 39.15 & 39.16 & 2.299 & 1 & 3 & 12 & 21 & 21 & 23 & 75.01 & 75.02 & 1.2652 & 111 & 1 & 13 & 11 & 34 \\
\hline 41.07 & 41.08 & 2.196 & 0 & 6 & 1 & 6 & 6 & 8 & 75.20 & 75.22 & 1.2625 & 012 & 1 & 1 & 7 & 3 \\
\hline 45.20 & 45.20 & 2.004 & 0 & 8 & 02 & 24 & 23 & 32 & 75.54 & & 1.2577 & 22 & 2 & 0 & & 1 \\
\hline 45.44 & 45.30 & 1.9945 & 1 & 5 & 1 & 1 & 15 & 2 & 77.37 & 77.36 & 1.2324 & 010 & 2 & 3 & 3 & 9 \\
\hline 47.14 & 47.14 & 1.9263 & 0 & 0 & 23 & 33 & 32 & 47 & 77.72 & 77.72 & 1.2277 & 28 & 1 & 3 & 3 & 8 \\
\hline 47.87 & 47.86 & 1.8988 & 1 & 7 & 02 & 29 & 27 & 42 & 78.08 & 78.08 & 1.2230 & 04 & 3 & 4 & 4 & 12 \\
\hline 48.57 & 48.56 & 1.8730 & 0 & 2 & 2 & 1 & 1 & 2 & 78.27 & 78.30 & 1.2205 & 19 & 2 & 0 & 2 & 1 \\
\hline 51.34 & 51.34 & 1.7782 & 0 & 8 & 1 & 6 & 6 & 10 & 78.79 & 78.80 & 1.2137 & 24 & 2 & 1 & 1 & 3 \\
\hline 52.67 & 52.68 & 1.7363 & 0 & 4 & 2 & 2 & 2 & 3 & 80.05 & 80.04 & 1.1978 & 11 & 3 & 6 & 5 & 17 \\
\hline 53.78 & 53.78 & 1.7032 & 1 & 7 & 12 & 20 & 20 & 34 & 82.19 & 82.18 & 1.1719 & 13 & 3 & 2 & 2 & 6 \\
\hline 53.98 & 53.98 & 1.6973 & 2 & 0 & 02 & 20 & 24 & 34 & 82.73 & 82.74 & 1.1656 & 210 & 0 & 2 & 2 & 7 \\
\hline 55.07 & 55.08 & 1.6663 & 1 & 1 & 2 & 8 & 8 & 15 & 83.44 & 83.44 & 1.1575 & 06 & 3 & 1 & 1 & 2 \\
\hline 55.28 & 55.22 & 1.6605 & 2 & 2 & 0 & 1 & 5 & 1 & 84.14 & 84.14 & 1.1496 & 26 & 2 & 0 & 0 & 1 \\
\hline 57.42 & 57.42 & 1.6035 & 01 & 10 & 0 & 5 & 6 & 9 & 86.07 & 86.08 & 1.1287 & 31 & 0 & 1 & 0 & 2 \\
\hline 57.61 & 57.60 & 1.5987 & 1 & 3 & 21 & 16 & 16 & 29 & 87.87 & 87.88 & 1.1101 & 113 & 1 & 0 & 0 & 0 \\
\hline 58.45 & 58.46 & 1.5776 & 1 & 9 & 0 & 1 & 1 & 2 & 88.19 & 88.20 & 1.1070 & 33 & 0 & 2 & 2 & 6 \\
\hline 59.06 & 59.06 & 1.5629 & 2 & 4 & 0 & 1 & 1 & 3 & 88.92 & 88.94 & 1.0997 & 111 & 2 & 1 & 1 & 4 \\
\hline 59.07 & & 1.5627 & 0 & 6 & 2 & 0 & & 0 & 89.11 & 89.12 & 1.0980 & 012 & 2 & 1 & 3 & 5 \\
\hline 62.49 & 62.50 & 1.4850 & 1 & 5 & 2 & 5 & 4 & 10 & 89.11 & & 1.0979 & 014 & 1 & 2 & & 7 \\
\hline 62.71 & 62.66 & 1.4804 & 01 & 10 & 1 & 0 & 3 & 0 & & & & & & & & \\
\hline 63.69 & 63.70 & 1.4599 & 1 & 9 & 1 & 2 & 2 & 5 & & & & & & & & \\
\hline 64.26 & 64.26 & 1.4483 & 2 & 4 & 11 & 16 & 14 & 34 & & & & & & & & \\
\hline
\end{tabular}


APPENDIX E: $\mathrm{Ca}\left(0,14 \mathrm{Sr}_{(0,86} \mathrm{CuO}_{2}\right.$ 
Table E.1. Simulated X-ray powder pattern for $\mathrm{Ca}_{0,14} \mathrm{Sr}_{(0,86} \mathrm{CuO}_{2}$

\begin{tabular}{|c|c|c|c|c|c|c|c|c|c|c|c|c|c|c|c|c|c|}
\hline $2 \theta$ & PEAK & d & $h$ & $k$ & $\operatorname{l111}$ & $|N T|$ & $(\mathrm{PK}) \|$ & (DS) & $2 \theta$ & PEAK & d & $h$ & k & 1111 & NT)! & $\overline{P K) I}$ & $\overline{(D S)}$ \\
\hline 23.02 & 23.02 & 3.861 & 1 & 0 & 0 & 2 & 2 & 1 & 62,83 & 62.82 & 1.4779 & 1 & 0 & 2 & 0 & 0 & 1 \\
\hline 27.86 & 27.88 & 3.200 & 0 & 0 & 1 & 11 & 11 & 8 & 67.85 & 67.84 & 1.3803 & 1 & 1 & 2 & 8 & 7 & 18 \\
\hline 32.78 & 32.78 & 2.730 & 1 & 1 & 0 & 31 & 32 & 27 & 68.70 & 68.70 & 1.3651 & 2 & 2 & 0 & 9 & 8 & 22. \\
\hline 36.44 & 36.44 & 2.464 & 1 & 0 & 11 & 1001 & 100 & 100 & 75.69 & 75.68 & 1.2556 & 2 & 2 & 1 & 1 & 1 & 3 \\
\hline 43.54 & 43.54 & 2.077 & 1 & 1 & 1 & 1 & 1 & 2 & 77.42 & 77.42 & 1.2318 & 2 & 0 & 2 & 12 & 10 & 34 \\
\hline 47.03 & 47.04 & 1.9305 & 2 & 0 & 0 & 37 & 34 & 51 & 78.23 & 78.24 & 1.2210 & 3 & 1 & 0 & 5 & 4 & 14 \\
\hline 52.99 & 52.98 & 1.7267 & 2 & 1 & 0 & 1 & 0 & 1 & 80.35 & 80.34 & 1.1940 & 3 & 0 & 1 & 7 & 6 & 22 \\
\hline 55.55 & 55.56 & 1.6530 & 2 & 0 & 1 & 4 & 3 & 7 & 82.05 & 82.06 & 1.1735 & 2 & 1 & 2 & 0 & 0 & 1 \\
\hline 57.57 & 57.58 & 1.5998 & 0 & 0 & 2 & 9 & 8 & 16 & 84.95 & 84.94 & 1.1407 & 3 & 1 & .1 & 0 & 0 & 0 \\
\hline 60.92 & 60.92 & 1.5196 & 2 & 1 & 1 & 37 & 33 & 75 & & & & & & & & & \\
\hline
\end{tabular}


APPENDIX F: $(\mathrm{Ca}, \mathrm{Sr}) \mathrm{Cu}_{2} \mathrm{O}_{3}$ 
Table F.1. Simulated X-raly powder pattern for $\mathrm{CaCl}_{2} \mathrm{O}_{3}$ Model 1

\begin{tabular}{|c|c|c|c|c|c|c|c|c|c|c|c|c|c|c|c|c|c|}
\hline \multirow{2}{*}{$\begin{array}{l}20 \\
17.83\end{array}$} & \multirow{2}{*}{$\frac{\text { PEAK }}{17.84}$} & \multirow{2}{*}{$\frac{d}{4.971}$} & \multirow{2}{*}{$\frac{h}{2}$} & \multirow{2}{*}{$\frac{k}{0}$} & \multicolumn{4}{|c|}{ l|(INT)|(PK)\|(DS) } & \multirow{2}{*}{$\frac{2 \theta}{66.59}$} & \multirow{2}{*}{$\frac{\text { PEAK }}{66.60}$} & \multirow{2}{*}{$\frac{d}{1.4032}$} & \multirow{2}{*}{$\frac{n}{6}$} & \multirow{2}{*}{$\frac{k}{1}$} & \multicolumn{4}{|c|}{$\Leftrightarrow|(\mid N T)|(P K) \mid(D S)$} \\
\hline & & & & & 0 & 6 & 7 & 3 & & & & & & 1 & 13 & 11 & 27 \\
\hline 23.56 & 23.56 & 3.773 & 1 & 1 & 0 & 6 & 7 & 4 & 69.76 & 70.14 & 1.3470 & 1 & 3 & 0 & 0 & 6 & 0 \\
\hline 25.73 & 25.74 & 3,459 & 0 & 0 & 1 & 16 & 17 & 10 & 70.10 & & 1.3413 & 7 & 1 & 0 & 0 & 0 & \\
\hline 27.28 & 27.28 & 3.267 & 1 & 0 & 1 & 22 & 23 & 15 & 70.13 & & 1.3407 & 4 & 1 & 2 & 7 & 15 & \\
\hline 31.48 & 31.40 & 2.839 & 2 & 0 & 1 & 62 & 63 & 51 & 71.46 & 71.46 & 1.3190 & 0 & 2 & 2 & 1 & 1 & 2 \\
\hline 33.96 & 33.96 & 2.638 & 0 & 1 & 1 & 88 & 91 & 79 & 71.62 & 71.64 & 1.3165 & 5 & 2 & 1 & 1 & 1 & 2 \\
\hline 34.85 & 34.86 & 2.572 & 3 & 1 & 0 & 72 & 74 & 66 & 71.79 & 71.80 & 1.3138 & 7 & 0 & 1 & 0 & 1 & 1 \\
\hline 35.17 & 35.16 & 2.550 & 1 & 1 & 1 & 14 & 18 & 13 & 72.19 & 72.20 & 1.3076 & 1 & 2 & $?$ & 6 & 5 & 13 \\
\hline 36.11 & 36.12 & 2.486 & 4 & 0 & 0 & 1 & 1 & 1 & 72.36 & 72.38 & 1.3049 & 5 & 0 & 2 & 3 & 5 & 7 \\
\hline 37.56 & 37.56 & 2.393 & 3 & 0 & 11 & 1001 & 1001 & 100 & 73.60 & 73.60 & 1.2860 & 6 & 2 & 0 & 11 & 9 & 26 \\
\hline 38.61 & 38.60 & 2.330 & 2 & 1 & 1 & 0 & 1 & 0 & 74.34 & 74.34 & 1.2749 & 2 & 2 & 2 & 0 & 0 & 1 \\
\hline 43.83 & 43.84 & 2.064 & 3 & 1 & 1 & 4 & 5 & 5 & 75.00 & 75,00 & 1.2653 & 0 & 3 & 1 & 4 & 3 & 9 \\
\hline 44.39 & 44.38 & 2.039 & 0 & 2 & 0 & 54 & 52 & 67 & 75.53 & 75.52 & 1.2578 & 3 & 3 & 0 & 4 & 3 & 9 \\
\hline 44.87 & 44.88 & 2.018 & 4 & 0 & 1 & 16 & 17 & 20 & 75.71 & 75.74 & 1.2552 & 1 & 3 & 1 & 1 & 2 & 2 \\
\hline 48.19 & 48.20 & 1.8867 & 2 & 2 & 0 & 1 & 1 & 1 & 76.04 & 76.04 & 1.2506 & 7 & 1 & 1 & 1 & 1 & 2 \\
\hline 51.06 & 51.06 & 1.7873 & 5 & 1 & 0 & 0 & 0 & 0 & 77.89 & 77.88 & 1.2255 & 3 & 2 & 2 & 4 & 3 & 11 \\
\hline 52.01 & 52.02 & 1.7567 & 0 & 2 & 1 & 3 & 3 & 4 & 79.44 & 79.44 & 1.2054 & 6 & 2 & 1 & 1 & 1 & 2 \\
\hline 52.88 & 52.90 & 1.7299 & 1 & 2 & 1 & 3 & 5 & 5 & 80.15 & 80.16 & 1.1965 & 6 & 0 & 2 & 1 & 0 & 1 \\
\hline 52.90 & & 1.7295 & 0 & 0 & 2 & 2 & 3 & & 81.33 & 81.34 & 1.1821 & 3 & 3 & 1 & 0 & 0 & 1 \\
\hline 53.08 & 53.04 & 1.7239 & 5 & 0 & 1 & 2 & 4 & 3 & 82.39 & 82.40 & 1.1696 & 8 & 0 & 1 & 1 & 1 & 3 \\
\hline 53.75 & 53.76 & 1.7039 & 1 & 0 & 2 & 11 & 10 & 17 & 82.77 & 82.64 & 1.1651 & 4 & 2 & 2 & 0 & 1 & 1 \\
\hline 55.40 & 55.42 & 1.6570 & 6 & 0 & 0 & 21 & 30 & 34 & 83.84 & 83.84 & 1.1530 & 0 & 0 & 3 & 1 & 1 & 2 \\
\hline 55.43 & & 1.6563 & 2 & 2 & 1 & 13 & 22 & & 84.28 & 84.28 & 1.1481 & 6 & 1 & 2 & 0 & 0 & 1 \\
\hline 56.27 & 56.28 & 1.6335 & 2 & 0 & 2 & 1 & 1 & 1 & 86.60 & 86.60 & 1.1232 & 2 & 0 & 3 & 2 & 1 & 5 \\
\hline 57.86 & 57.88 & 1.5923 & 0 & 1 & 2 & 1 & 1 & 1 & 87.94 & 87.94 & 1.1095 & 0 & 1 & 3 & 0 & 0 & 0 \\
\hline 58.04 & 58.04 & 1.5879 & 5 & 1 & 1 & 2 & 2 & 4 & 88.44 & 88.62 & 1.1045 & 7 & 2 & 1 & 0 & 1 & 1 \\
\hline 58.50 & 58.50 & 1.5766 & 4 & 2 & 0 & 0 & 0 & 0 & 88.63 & & 1.1027 & 1 & 1 & 3 & 2 & 5 & \\
\hline 58.67 & 58.68 & 1.5722 & 1 & 1 & 2 & 0 & 1 & 1 & 88.98 & 88.98 & 1.0992 & 5 & 2 & 2 & 2 & 2 & 7 \\
\hline 59.51 & 59.52 & 1.5521 & 3 & 2 & 1 & 31 & 27 & 56 & 89.14 & 89.14 & 1.0976 & 7 & 0 & 2 & 1 & 1 & 3 \\
\hline 60.32 & 60.32 & 1.5333 & 3 & 0 & 2 & 8 & 7 & 14 & & & & & & & & & \\
\hline 61.06 & 61.06 & 1.5164 & 2 & 1 & 2 & 11 & 10 & 21 & & & & & & & & & \\
\hline 62.06 & 62.06 & 1.4944 & 6 & 0 & 1 & 1 & 1 & 2 & & & & & & & & & \\
\hline 64.92 & 64.94 & 1.4352 & 3 & 1 & 2 & 4 & 8 & 7 & & & & & & & & & \\
\hline 64.95 & & 1.4346 & 4 & 2 & 1 & 6 & 12 & & & & & & & & & & \\
\hline 65.72 & 65.72 & 1.4196 & 4 & 0 & 2 & 0 & 0 & 1 & & & & & & & & & \\
\hline
\end{tabular}


Table F.2. Simulated X-raly powder pattern for $\mathrm{CaCu}_{2} \mathrm{O}_{3}$ Model 2

\begin{tabular}{|c|c|c|c|c|c|c|c|c|c|c|c|c|c|c|c|c|c|}
\hline \multirow{2}{*}{$-\frac{2 \theta}{17.83}$} & \multirow{2}{*}{$\frac{\text { PEAK }}{17.84}$} & \multirow{2}{*}{$\frac{d}{4.971}$} & \multirow{2}{*}{$\frac{h}{2}$} & \multirow{2}{*}{$\frac{k}{0}$} & \multicolumn{4}{|c|}{$\ell|(\mid N T)|(P K) \mid(D S)$} & \multirow{2}{*}{\begin{tabular}{|l|}
$2 \theta$ \\
70.13
\end{tabular}} & \multirow{2}{*}{$\frac{\text { PEAK }}{70.14}$} & \multirow{2}{*}{$\frac{d}{1.3407}$} & \multirow{2}{*}{$\frac{h}{4}$} & \multirow{2}{*}{$\frac{k}{1}$} & \multicolumn{4}{|c|}{ l $|(\mid N T)|(P K) \mid(D S)$} \\
\hline & & & & & 0 & 6 & 8 & 3 & & & & & & 2 & $\theta$ & 5 & 13 \\
\hline 23.56 & 23.56 & 3.773 & 1 & 1 & 0 & 4 & 4 & 2 & 71.46 & 71.46 & 1.3190 & 0 & 2 & 2 & 1 & 1 & 2 \\
\hline 25.73 & 25.74 & 3.459 & 0 & 0 & 1 & 12 & 14 & 8 & 71,62 & 71.64 & 1.3165 & 5 & 2 & 1 & 1 & 1 & 2 \\
\hline 27.28 & 27.28 & 3.267 & 1 & 0 & 1 & 22 & 24 & 16 & 71.79 & 71.82 & 1.3138 & 7 & 0 & 1 & 0 & 1 & 1 \\
\hline 31,48 & 31.48 & 2.839 & 2 & 0 & 1 & 58 & 59 & 47 & 72.19 & 72.20 & 1.3076 & 1 & 2 & 2 & 5 & 5 & 13 \\
\hline 33.96 & 33.96 & 2.638 & 0 & 1 & 1 & 73 & 75 & 65 & 72.36 & 72.38 & 1.3049 & 5 & 0 & 2 & 3 & 5 & 7 \\
\hline 34.85 & 34.86 & 2.572 & 3 & 1 & 0 & 69 & 72 & 64 & 73.60 & 73.60 & 1.2860 & 6 & 2 & 0 & 10 & 9 & 25 \\
\hline 35.17 & 35.16 & 2.550 & 1 & 1 & 1 & 17 & 21 & 15 & 74.34 & 74.34 & 1.2749 & 2 & 2 & 2 & 0 & 0 & 1 \\
\hline 36.11 & 36.12 & 2.486 & 4 & 0 & 0 & 1 & 1 & 0 & 75.00 & 75.00 & 1.2653 & 0 & 3 & 1 & 3 & 3 & 8 \\
\hline 37.56 & 37.56 & 2.393 & 3 & 0 & 1 & 100 & 1001 & 100 & 75.53 & 75.52 & 1.2578 & 3 & 3 & 0 & 4 & 3 & 9 \\
\hline 43.83 & 43.84 & 2.064 & 3 & 1 & 1 & 8 & 8 & 10 & 75.71 & 75.74 & 1.2552 & 1 & 3 & 1 & 1 & 2 & 2 \\
\hline 44.39 & 44.38 & 2.039 & 0 & 2 & 0 & 53 & 50 & 65 & 76.04 & 76.04 & 1.2506 & 7 & 1 & 1 & 1 & 1 & 2 \\
\hline 44.87 & 44.88 & 2.018 & 4 & 0 & 1 & 15 & 15 & 18 & 77.89 & 77.88 & 1.2255 & 3 & 2 & 2 & 4 & 3 & 10 \\
\hline 48.19 & 48.20 & 1.8867 & 2 & 2 & 0 & 1 & 1 & 1 & 79.44 & 79.44 & 1.2054 & 6 & 2 & 1 & 1 & 0 & 2 \\
\hline 50.40 & 50.40 & 1.8090 & 4 & 1 & 1 & 0 & 0 & 1 & 80.15 & 80.16 & 1.1965 & 6 & 0 & 2 & 1 & 0 & 2 \\
\hline 51.06 & 51.06 & 1.7873 & 5 & 1 & 0 & 1 & 1 & 2 & 81.33 & 81.34 & 1.1821 & 3 & 3 & 1 & 0 & 0 & 1 \\
\hline 52.01 & 52.02 & 1.7567 & 0 & 2 & 1 & 2 & 2 & 4 & 82.39 & 82.40 & 1.1696 & 8 & 0 & 1 & 1 & 1 & 3 \\
\hline 52.88 & 52.90 & 1.7299 & 1 & 2 & 1 & 3 & 5 & 5 & 82.77 & 82.64 & 1.1651 & 4 & 2 & 2 & 0 & 1 & 1 \\
\hline 52.90 & & 1.7295 & 0 & 0 & 2 & 2 & & 3 & 83.84 & 83.84 & 1.1530 & 0 & 0 & 3 & 1 & 1 & 2 \\
\hline 53.08 & 53.04 & 1.7239 & 5 & 0 & 1 & 2 & 4 & 3 & 84.28 & 84.28 & 1.1481 & 6 & 1 & 2 & 0 & 0 & 1 \\
\hline 53.75 & 53.76 & 1.7039 & 1 & 0 & 2 & 10 & 9 & 16 & 86.60 & 86.60 & 1.1232 & 2 & 0 & 3 & 2 & 1 & 5 \\
\hline 55.40 & 55.42 & 1.6570 & 6 & 0 & 0 & 20 & 29 & 33 & 87.94 & 87.94 & 1.1095 & 0 & 1 & 3 & 1 & 0 & 2 \\
\hline 55.43 & & 1.6563 & 2 & 2 & 1 & 13 & & 21 & 88.44 & 88.62 & 1.1045 & 7 & 2 & 1 & 0 & 1 & 0 \\
\hline 56.27 & 56.28 & 1.6335 & 2 & 0 & 2 & 1 & 1 & 1 & 88.63 & & 1.1027 & 1 & 1 & 3 & 1 & & 4 \\
\hline 57.86 & 57.88 & 1.5923 & 0 & 1 & 2 & 1 & 1 & 2 & 88.98 & 88.98 & 1.0992 & 5 & 2 & 2 & 2 & 2 & 7 \\
\hline 58.04 & 58.04 & 1.5879 & 5 & 1 & 1 & 4 & 4 & 7 & 89.14 & 89.14 & 1.0976 & 7 & 0 & 2 & 1 & 1 & 3 \\
\hline 58.50 & 58.48 & 1.5766 & 4 & 2 & 0 & 0 & 0 & 0 & & & & & & & & & \\
\hline 59.51 & 59.52 & 1.5521 & 3 & 2 & 1 & 31 & 27 & 55 & & & & & & & & & \\
\hline 60.32 & 60.32 & 1.5333 & 3 & 0 & 2 & 7 & 7 & 14 & & & & & & & & & \\
\hline 61.06 & 61.06 & 1.5164 & 2 & 1 & 2 & 14 & 12 & 25 & & & & & & & & & \\
\hline 62.06 & 62.06 & 1.4944 & 6 & 0 & 1 & 1 & 1 & 2 & & & & & & & & & \\
\hline 64.92 & 64.94 & 1.4352 & 3 & 1 & 2 & 1 & 6 & 3 & & & & & & & & & \\
\hline 64.95 & & 1.4346 & 4 & 2 & 1 & 6 & & 11 & & & & & & & & & \\
\hline 65.72 & 65.72 & 1.4196 & 4 & 0 & 2 & 0 & 0 & 1 & & & & & & & & & \\
\hline 66.59 & 66.60 & 1.4032 & 6 & 1 & 1 & 11 & 10 & 23 & & & & & & & & & \\
\hline
\end{tabular}


Table F.3. Simulated X-ray powder pattern for $\mathrm{CaCu}_{2} \mathrm{O}_{3}$ Model 3

\begin{tabular}{|c|c|c|c|c|c|c|c|c|c|c|c|c|c|c|c|c|c|}
\hline $2 \theta$ & PEAK & d & h & $k$ & $\operatorname{ll} 1$ & NTTI & $(\mathrm{PK}) \mathrm{I}$ & I(DS) & $2 \theta$ & PEAK & d & $h$ & $k$ & & NT & $P K$ & S) \\
\hline 17.83 & 17.84 & 4.971 & 2 & 0 & 0 & 2 & 3 & 1 & 60.32 & 60.32 & 1.5333 & 3 & 0 & 2 & 5 & 5 & 10 \\
\hline 23.56 & 23.56 & 3.773 & 1 & 1 & 0 & 4 & 4 & 2 & 61.06 & 61.06 & 1.5164 & 2 & 1 & 2 & 9 & 8 & 17 \\
\hline 25.73 & 25.74 & 3.459 & 0 & 0 & 1 & 26 & 28 & 17 & 62.06 & 62.06 & 1.4944 & 6 & 0 & 1 & 2 & 1 & 3 \\
\hline 27.28 & 27.28 & 3.267 & 1 & 0 & 1 & 5 & 5 & 3 & 64.92 & 64.94 & 1.4352 & 3 & 1 & 2 & 2 & 3 & 3 \\
\hline .31 .48 & 31.48 & 2.839 & 2 & 0 & 1 & 17 & 18 & 14 & 64.95 & & 1.4346 & 4 & 2 & 1 & 3 & & 5 \\
\hline 33.96 & 33.96 & 2.638 & 0 & 1 & 1 & 39 & 40 & 35 & 65.72 & 65.72 & 1.4196 & 4 & 0 & 2 & 0 & 0 & 1 \\
\hline 34.85 & 34.86 & 2.572 & 3 & 1 & 0 & 53 & 55 & 48 & 66.59 & 66.60 & 1.4032 & 6 & 1 & 1 & 7 & 6 & 14 \\
\hline 35.17 & 35.18 & 2.550 & 1 & 1 & 1 & 34 & 37 & 31 & 70.13 & 70.14 & 1.3407 & 4 & 1 & 2 & 5 & 4 & 11 \\
\hline 36.11 & 36.12 & 2.486 & 4 & 0 & 0 & 1 & 2 & 1 & 71.46 & 71.46 & 1.3190 & 0 & 2 & 2 & 1 & 1 & 2 \\
\hline 37.56 & 37.56 & 2.393 & 3 & 0 & 1 & .00 & 100 & 100 & 71.62 & 71.66 & 1.3165 & 5 & 2 & 1 & 0 & 1 & 0 \\
\hline 38.61 & 38.60 & 2.330 & 2 & 1 & 1 & 2 & 2 & 2 & 71.79 & & 1.3138 & 7 & 0 & 1 & 0 & & 0 \\
\hline 43.83 & 43.84 & 2.064 & 3 & 1 & 1 & 0 & 1 & 0 & 72.19 & 72.20 & 76 & 1 & 2 & 2 & 4 & 4 & 9 \\
\hline 44.39 & 44.38 & 2.039 & 0 & 2 & 0 & 40 & 38 & 49 & 72.36 & 72.38 & 49 & 5 & 0 & 2 & - & 3 & 4 \\
\hline 44.87 & 44.88 & 2.018 & 4 & 0 & 1 & 6 & 7 & 7 & 73.60 & 73.50 & 1.2860 & 6 & 2 & 0 & 8 & 7 & 19 \\
\hline 48.19 & 48.20 & 1.8867 & 2 & 2 & 0 & 0 & 0 & 0 & 74.34 & 74.34 & 1.2749 & 2 & 2 & 2 & 0 & 0 & 1 \\
\hline 50.40 & 50.40 & 1.8090 & 4 & 1 & 1 & 0 & 0 & 1 & 75.00 & 75.00 & 1.2653 & 0 & 3 & 1 & & & 5 \\
\hline 51.06 & 51.06 & 1.7873 & 5 & 1 & 0 & 0 & 0 & 1 & 75.53 & 75.54 & 1.2578 & 3 & 3 & 0 & 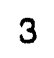 & 3 & 7 \\
\hline 52.01 & 52.02 & 1.7567 & 0 & 2 & 1 & 4 & 3 & 6 & 75.71 & 75.74 & 1.2552 & 1 & 3 & 1 & 1 & 2 & 3 \\
\hline 52.88 & 52.90 & 1.7299 & 1 & 2 & 1 & 1 & 3 & 1 & 76.04 & 76.04 & 1.2506 & 7 & 1 & 1 & 1 & 1 & 3 \\
\hline 52.90 & & 1.7295 & 0 & $c$ & 2 & 2 & & 3 & 77.89 & 77.88 & 1.2255 & 3 & 2 & 2 & 3 & 2 & 7 \\
\hline 53.08 & 53.02 & 1.7239 & 5 & 0 & 1 & 0 & 2 & 0 & 44 & 79.44 & 54 & 6 & 2 & 1 & 1 & 1 & 2 \\
\hline 53.75 & 53.76 & 1.7039 & 1 & 0 & 2 & 8 & 7 & 12 & 80.15 & 80.16 & 1.1965 & 6 & 0 & 2 & 1 & 1 & 2 \\
\hline 55.40 & 55.42 & 1.6570 & 6 & 0 & 0 & 15 & 17 & 25 & 82.39 & 82.40 & 1.1696 & 8 & 0 & 1 & 0 & 0 & 1 \\
\hline 55.43 & & 1.6563 & 2 & 2 & 1 & 5 & & 7 & 82.77 & 82.76 & 1.1651 & 4 & 2 & 2 & 0 & 0 & 1 \\
\hline 56.27 & 56.28 & 1.6335 & 2 & 0 & 2 & 1 & 1 & 1 & 83.84 & 83.84 & 1.1530 & 0 & 0 & 3 & 1 & 1 & 2 \\
\hline 57.86 & 58.04 & 1.5923 & 0 & 1 & 2 & 1 & 5 & 1 & 84.28 & 84.28 & 1.1481 & 6 & 1 & 2 & 0 & 0 & 0 \\
\hline 58.04 & & 1.5879 & 5 & 1 & 1 & 5 & & 9 & 86.60 & 86.60 & 1.1232 & 2 & 0 & 3 & 1 & $i$ & 3 \\
\hline 58.50 & 58.50 & 1.5766 & 4 & 2 & 0 & 0 & 1 & 1 & 88.63 & 88.62 & 1.1027 & 1 & 1 & 3 & 1 & 1 & 4 \\
\hline 58.67 & 58.66 & 1.5722 & 1 & 1 & 2 & 0 & 1 & 0 & 88.98 & 88.98 & 1.0992 & 5 & 2 & 2 & 1 & 2 & 4 \\
\hline 59.51 & 59.52 & 1.5521 & 3 & 2 & 1 & 30 & 26 & 53 & 89.14 & 89.14 & 1.0976 & 7 & $n$ & 2 & 1 & 1 & 3 \\
\hline
\end{tabular}


Table F.4. Simulated X-ray powder pattern for $\mathrm{CaCu}_{2} \mathrm{O}_{3}$ Model 4

\begin{tabular}{|c|c|c|c|c|c|c|c|c|c|c|c|c|c|c|c|c|c|}
\hline $2 \theta$ & PEAK & $d$ & $h$ & $k$ & $\operatorname{ll}$ & $|\mathrm{NT}|$ & $(\mathrm{PK})$ & (DS) & $2 \theta$ & PEAK & d & $h$ & $k$ & & NT) & FK) & (DS) \\
\hline 17.83 & 17.84 & 4.971 & 2 & 0 & 0 & 4 & 4 & 2 & 65.72 & 65.72 & 1.4196 & 4 & 0 & 2 & 0 & 0 & 0 \\
\hline 23.56 & 23.56 & 3.773 & 1 & 1 & 0 & 4 & 5 & 3 & 66.59 & 66.60 & 1.4032 & 6 & 1 & 1 & 7 & 6 & 15 \\
\hline 25.73 & 25.74 & 3.459 & 0 & 0 & 1 & 29 & 32 & 19 & 70.10 & 70.14 & 1.3413 & 7 & 1 & 0 & 0 & 4 & 0 \\
\hline 27.28 & 27.28 & 3.267 & 1 & 0 & 1 & 4 & 4 & 3 & 70.13 & & 1.3407 & 4 & 1 & 2 & 5 & & 11 \\
\hline 31.48 & 31.48 & 2.839 & 2 & 0 & 1 & 20 & 20 & 16 & 71.46 & 71.46 & 1.3190 & 0 & 2 & 2 & 1 & 1 & 2 \\
\hline 33.96 & 33.96 & 2.638 & 0 & 1 & 1 & 42 & 44 & 38 & 71.62 & 71.66 & 1.3165 & 5 & 2 & 1 & 0 & 1 & 0 \\
\hline 34.85 & 34.86 & 2.572 & 3 & 1 & 0 & 54 & 56 & 49 & 71.79 & & 1.3138 & 7 & 0 & 1 & 0 & & 0 \\
\hline 35.17 & 35.18 & 2.550 & 1 & 1 & 1 & 34 & 37 & 31 & 72.19 & 72.20 & 1.3076 & 1 & 2 & 2 & 4 & 4 & 10 \\
\hline 36.11 & 36.12 & 2.486 & 4 & 0 & 0 & 1 & 1 & 1 & 72.36 & 72.38 & 1.3049 & 5 & 0 & 2 & 2 & 4 & 5 \\
\hline 37.56 & 37.56 & 2.393 & 3 & 0 & 1 & 100 & 100 & 100 & 73.60 & 73.60 & 1.2860 & 6 & 2 & 0 & 8 & 7 & 20 \\
\hline 38.61 & 38.60 & 2.330 & 2 & 1 & 1 & 1 & 2 & 2 & 74.34 & 74.34 & 1.2749 & 2 & 2 & 2 & 0 & 0 & 1 \\
\hline 43.83 & 44.38 & 2.064 & 3 & 1 & 1 & 0 & 39 & 0 & 75.00 & 75.00 & 1.2653 & 0 & 3 & $y$ & 2 & 2 & 5 \\
\hline 44.39 & & 2.039 & 0 & 2 & 0 & 41 & & 50 & 75.53 & 75.54 & 1.2578 & 3 & 3 & 0 & 3 & 3 & 7 \\
\hline 44.87 & 44.88 & 2.018 & 4 & 0 & 1 & 6 & 7 & 8 & 75.71 & 75.74 & 1.2552 & 1 & 3 & 1 & 1 & 2 & 3 \\
\hline 48.19 & 48.20 & 1.8867 & 2 & 2 & 0 & 0 & 0 & 1 & 76.04 & 76.04 & 1.2506 & 7 & 1 & 1 & 1 & 2 & 4 \\
\hline 50.40 & 50.40 & 1.8090 & 4 & 1 & 1 & 1 & 1 & 1 & 77.89 & 77.88 & 1.2255 & 3 & 2 & 2 & 3 & 3 & 8 \\
\hline 51.06 & 51.06 & 1.7873 & 5 & 1 & 0 & 0 & 0 & 0 & 79.44 & 79.44 & 1.2054 & 6 & 2 & 1 & 1 & 1 & 3 \\
\hline 52.01 & 52.02 & 1.7567 & 0 & 2 & 1 & 4 & 4 & 6 & 80.15 & 80.16 & 1.1965 & 6 & 0 & 2 & 1 & 0 & 1 \\
\hline 52.88 & 52.90 & 1.7299 & 1 & 2 & 1 & 1 & 3 & 1 & 82.39 & 82.40 & 1.1696 & 8 & 0 & 1 & 0 & 0 & 1 \\
\hline 52.90 & & 1.7295 & 0 & 0 & 2 & 2 & & 3 & 82.77 & 82.76 & 1.1651 & 4 & 2 & 2 & 0 & 0 & 0 \\
\hline 53.08 & 53.04 & 1.7239 & 5 & 0 & 1 & 0 & 2 & 1 & 83.84 & 83.84 & 1.1530 & 0 & 0 & 3 & 1 & 1 & 2 \\
\hline 53.75 & 53.76 & 1.7039 & 1 & 0 & 2 & 8 & 7 & 13 & 84.28 & 84.28 & 1.1481 & 6 & 1 & 2 & 0 & 0 & 0 \\
\hline 55.40 & 55.42 & 1.6570 & 6 & 0 & 0 & 16 & 18 & 26 & 86.60 & 86.60 & 1.1232 & 2 & 0 & 3 & 1 & 1 & 3 \\
\hline 55.43 & & 1.6563 & 2 & 2 & 1 & 5 & & 8 & 88.63 & 88.62 & 1.1027 & 1 & 1 & 3 & 1 & 1 & 5 \\
\hline 56.27 & 56.28 & 1.6335 & 2 & 0 & 2 & 0 & 1 & 1 & 88.98 & 88.98 & 1.0992 & 5 & 2 & 2 & 2 & 2 & 5 \\
\hline 57.86 & 58.04 & 1.5923 & 0 & 1 & 2 & 0 & 4 & 1 & 89.14 & 89.14 & 1.0976 & 7 & 0 & 2 & 1 & 1 & 3 \\
\hline 58.04 & & 1.5879 & 5 & 1 & 1 & 4 & & 8 & & & & & & & & & \\
\hline 58.50 & 58.50 & 1.5766 & 4 & 2 & 0 & 0 & 1 & 0 & & & & & & & & & \\
\hline 58.67 & 58.66 & 1.5722 & 1 & 1 & 2 & 0 & 1 & 0 & & & & & & & & & \\
\hline 59.51 & 59.52 & 1.5521 & 3 & 2 & 1 & 30 & 26 & 54 & & & & & & & & & \\
\hline 60.32 & 60.32 & 1.5333 & 3 & 0 & 2 & 6 & 6 & 11 & & & & & & & & & \\
\hline 61.06 & 61.06 & 1.5164 & 2 & 1 & 2 & 9 & 8 & 17 & & & & & & & & & \\
\hline 62.06 & 62.06 & 1.4944 & 6 & 0 & 1 & 2 & 2 & 3 & & & & & & & & & \\
\hline 64.92 & 64.94 & 1.4352 & 3 & 1 & 2 & 2 & 4 & 4 & & & & & & & & & \\
\hline 64.95 & & 1.4346 & 4 & 2 & 1 & 3 & & 5 & & & & & & & & & \\
\hline
\end{tabular}


APPENDIX G: $(\mathrm{Ca}, \mathrm{Sr}) \mathrm{Cu}_{2} \mathrm{O}_{2}$ 
Table G.1. Simulated X-ray powder pattern for $\mathrm{SrCu}_{2} \mathrm{O}_{2}$

\begin{tabular}{|c|c|c|c|c|c|c|c|c|c|c|c|c|c|c|c|c|c|}
\hline $2 \theta$ & $\overline{\text { PEAK }}$ & d & $\bar{h}$ & $\bar{k}$ & l|IIN & $\sqrt{T) \mid(F}$ & $\overline{\mathrm{PK}) !(\mathrm{L}}$ & (DS) & $2 \theta$ & PEAK & d & $\bar{h}$ & $\bar{k}$ & $\overline{l \mid(\mathbb{N}}$ & $\sqrt{\operatorname{VT} 1(}$ & $\overline{\mathrm{PK}) \|(}$ & (DS) \\
\hline 18.55 & 18.56 & 4.779 & 1 & 0 & 11 & 19 & 21 & 11 & 61.84 & 61.84 & 1.4991 & 3 & 2 & 1 & 14 & 13 & 39 \\
\hline 29.37 & 29.38 & 3.039 & 1 & 1 & 210 & & 1001 & 100 & 66.50 & 66.50 & 1.4050 & 2 & 0 & 6 & 7 & 6 & 21 \\
\hline 31.82 & 31.82 & 2.810 & 1 & 0 & 35 & 52 & 51 & 57 & 68.06 & 68.08 & 1.3764 & 3 & 2 & 3 & 0 & 0 & 0 \\
\hline 32.72 & 32.72 & 2.734 & 2 & 0 & 03 & 36 & 35 & 41 & 68.58 & 68.58 & 1.3672 & 4 & 0 & 0 & 11 & 9 & 36 \\
\hline $3^{-} .62$ & 37.62 & 2.389 & 2 & 0 & 25 & 50 & 51 & 68 & 70.39 & 70.38 & 1.3365 & 3 & 0 & 5 & 5 & 4 & 15 \\
\hline 37.88 & 37.88 & 2.373 & 2 & 1 & 16 & 65 & 66 & 90 & 76.31 & 76.32 & 1.2469 & 3 & 3 & 2 & 3 & 3 & 13 \\
\hline 46.29 & 46.30 & 1.9597 & 2 & 1 & 3 & 1 & 1 & 2 & 77.59 & 77.60 & 1.2294 & 4 & $i$ & 3 & 7 & 6 & 25 \\
\hline 49.23 & 49.24 & 1.8494 & 1 & 0 & 51 & 15 & 13 & 30 & 77.68 & & 1.2283 & 0 & 0 & 8 & 4 & & 13 \\
\hline 49.86 & 49.86 & 1.8274 & 2 & 0 & 4 & 17 & 15 & 34 & 78.08 & 78.08 & 1.2229 & 4 & 2 & 0 & 3 & 3 & 12 \\
\hline 50.90 & 50.90 & 1.7924 & 3 & 0 & 1 & 0 & 0 & 1 & 78.50 & 78.50 & 1.2175 & 2 & 1 & 7 & 6 & 5 & 25 \\
\hline 56.35 & 56.36 & 1.6313 & 3 & 1 & 22 & 22 & 19 & 51 & 80.75 & 80.76 & 1.1891 & 3 & 1 & 6 & 6 & 5 & 23 \\
\hline 57.84 & 57.84 & 1.5929 & 3 & 0 & 3 & 9 & 8 & 22 & 80.95 & 80.96 & 1.1867 & 4 & 2 & '2 & 7 & 7 & 26 \\
\hline 60.37 & 60.40 & 1.5319 & 2 & 1 & 5 & 0 & 1 & 1 & 86.87 & 86.86 & 1.1204 & 2 & 0 & 8 & 2 & 2 & 10 \\
\hline 60.93 & 60.92 & 1.5194 & 2 & 2 & 4 & 35 & 30 & 94 & 88.96 & 88.96 & 1.0994 & 4 & 1 & 5 & 4 & 3 & 20 \\
\hline 61.43 & 361.44 & 1.5080 & 1 & 1 & 6 & 8 & 8 & 21 & 89.44 & 89.44 & 1.0948 & 4 & 2 & 4 & 4 & 3 & 20 \\
\hline
\end{tabular}


ORNL/TM-1 1948

\section{INTERNAL DISTRIBUTION}

1-2. Central Research Library

3. Document Reference Section

4-5. Laboratory Records Department

6. Laboratory Records, ORNL-RC

7. ORNL Patent Section

8-10. M\&C Records Office

11. R. S. Carlsmith

12. O. B. Cavin

13. D. K. Christen

14. D. F. Craig

15. L. B. Dunlap

16. F. Foust

17. J. D. Griffin

18. R. A. Hawsey

19-23. C. R. Hubbard

24. D. M. Kroeger
25. M. S. Lubell

26. B. W. McConnell

27. H. Payne

28-32. B. J. Reardon

33. J. B. Roberto

34. A. C. Schaffhauser

35. E. J. Soderstrom

36. E. D. Specht

37. V. J. Tennery

38. Z. L. Wang

39. Y. A. Chang (Consultant)

40. H. W. Foglesong (Consultant)

41. J. J. Hren (Consultant)

42. M. L.Savitz (Consultant)

43. J. G. Simon (Consultant)

44. K. E. Spear (Consultant)

\section{EXTERNAL DISTRIBUTION}

45. ALFRED UNIVERSITY, Binns-Merill Hall, Alfred, NY 14802

R. L. Snyder

46-47. ARGONNE NATIONAL LABORATORY, 9700 S. Cass Avenue, Argonne, IL 60439

S. Lake $($ TTC/900)

R. Poeppel (Bldg 212)

48. BROOKHAVEN NATIONAL LABORATORY, Department of Applied Science, MTLS Science Division, Building 480, Upton, Long Island, NY 11973

D. O. Welch

49. CORNING INCORPORATED, Research, Development \& Engineering, Instrumental Analysis Department, SP FR OI 8, Corning, NY 14831

H. J. Holland

50. EASTMAN KODAK COMPANY, Kodak Park, Building 49, Rochester, NY 14652-3712

T. N. Blanton 
51. ENERGETICS, INC., Project Manager, 7164 Columbia Gateway Drive, Columbia, MD 21046

J. Badin

52-54. LOS ALAMOS NATIONAL LABORATORY, P. O. Box 1663, Los Alamos, NM 87545

K. Alanks (MS K763)

L. S. Llair, Industrial Program Manager (ERDC/SPC, MS K763)

E. Peterson (MS K763)

55. MONASH UNIVERSITY, Department of Physics, Clayton 3168, A' 'stralia

R. A. Coyle

56-58. NATIONAL INSTITUTE OF STANDARDS AND TECHNOLOGY, Materials

Science \& Engineering Laboratory, Gaithersburg, MD 20899

L. P. Cook

R. S. Roth

W. Wong-Ng

59. NATIONAL RENEWABLE ENERGY LABORATORY, 1617 Cole Blvd., Golden, CO 80401

R. D. Blaugher

6(). NAVAL WEAPONS CENTER, Code 3851, China Lake, CA 93555

C. Lowe-Ma

61. SANDIA NATIONAL LABORATORIES, Division 6221, P. O. Box 5800, Albuquerque, NM 87185

T. C. Bickel

62. U. S. DOE, ADVANCED UTILITY CONCEPTS DIVISION, Director, Forrestal RM 5F064, MS CE142, 1000 Independence Avenue SW, Washington, DC 20585

R. Eaton

63-64. U. S. DOE, CONSERVATION AND RENEWABLE ENERGY, Forrestal Building, 1000 Independence Avenue, S. W., Washington, DC 20585

A. A. Chesnes, Deputy Assistant Secretary

J. J. Eberhardt 
65. U. S. DOE, SUPERCONDUCTIVITY SYSTEMS OIFICE OF ENERGY MANAGEMENT, Program Manager, Forrestal RM 5F(064, 100() Independence Avenue SW, Washington, DC 20585

J. Daley (MS CE142)

66-69. U. S. DOE, OAK RIDGE FIELD OFFICE, P. O. Box 2008, Oak Ridge, TN 37831-6269

Assistant Manager for Energy Research and Development

G. C. Manthey

G. L. Riner

M. J. Rohr

70)-79. U. S. DOE, OFFICE OF SCIENTIFIC AND TECHNICAL INFORMATION, P. O. Box 62, Oak Ridge, TN 37831

For distribution by microfiche as shown in DOE/OSTI-45(X), Distribution Category UC. 364 (Superconducting Materials) 

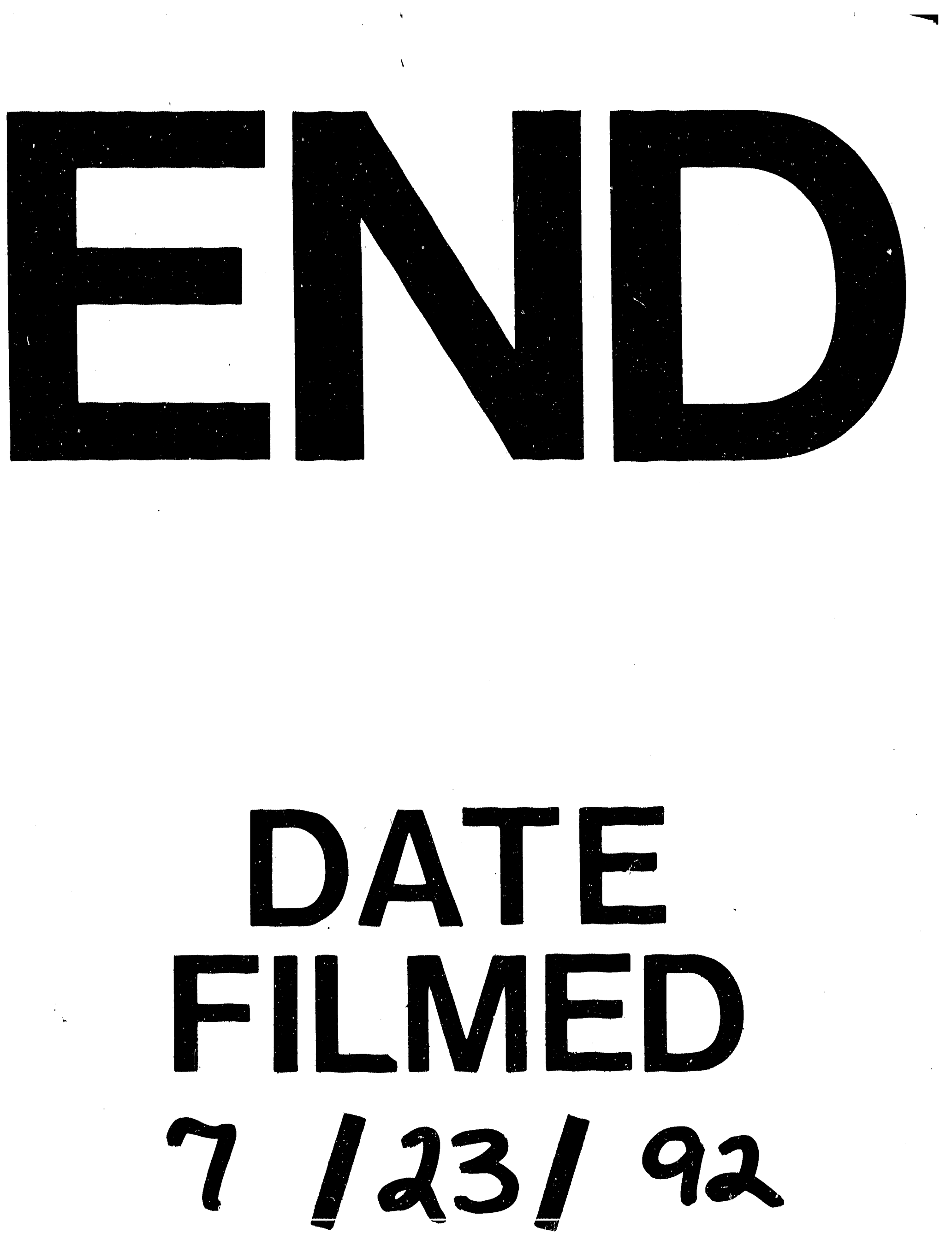
